LBL -31760

DE93 000463

\title{
Simulation of Tracer Transport for the Site Characterization and Validation Site in the Stripa Mine
}

\author{
Jane C. S. Long and K. Karasaki \\ Earth Sciences Division \\ Lawrence Berkeley Laboratory \\ University of California \\ Berkeley, California 94720
}

January 1992

This work was supported by the Office of Civilian Radioactive Waste Management, Office of Strategic Planning and Internal Programs, of the U.S. Department of Energy under Contract No. DE-AC03$76 S F 00098$.

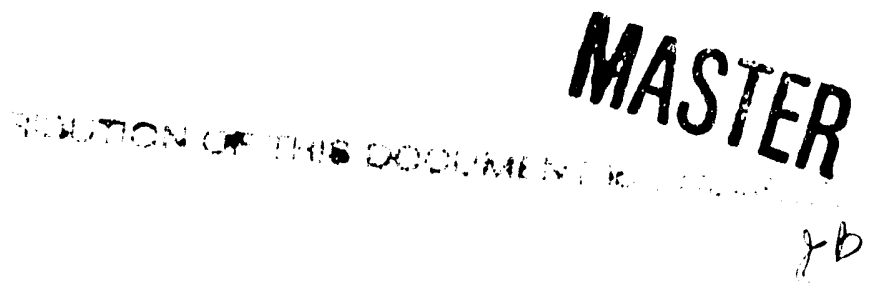




\section{Table of Contents}

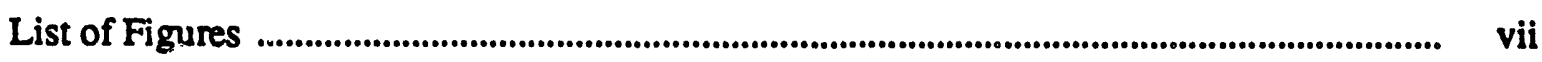

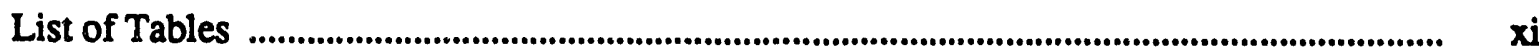

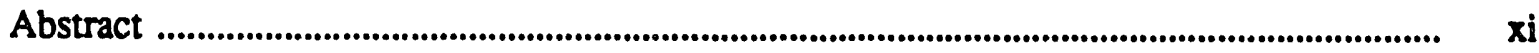

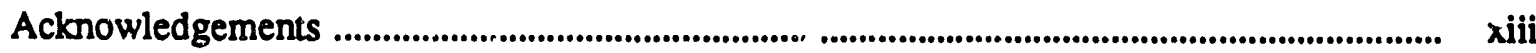

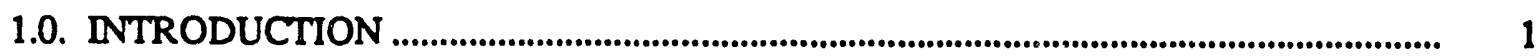

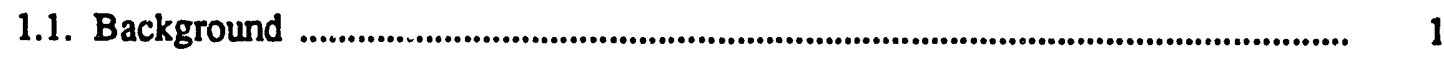

1.2. Modeling Approach .............................................................................................. 3

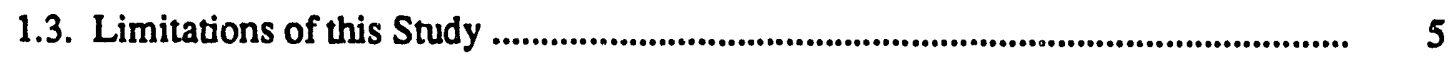

1.4. Structure of this Report ..................................................................................... 6

2.0. TWO-DIMENSIONAL MODELS USED FOR TRANSPORT

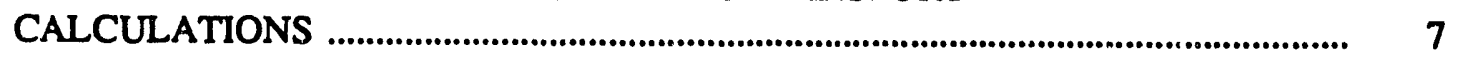

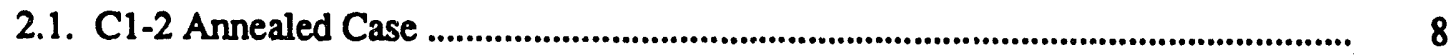

2.2. Co-Annealed Case .................................................................................................... 8

2.3. Altering the Models to Represent the Excavation of the Validation Drift ............ 13

3.0. SIMULATION OF TRACER TRANSPORT .............................................................. 15

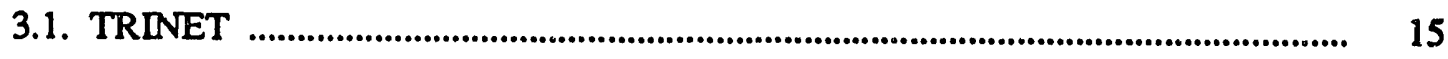

3.2. Parameters Needed to Model Transport .................................................................. 16

3.3. Flow Conditions ................................................................................................. 18

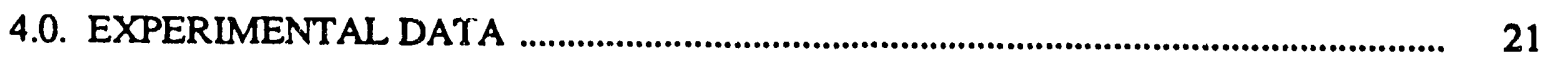

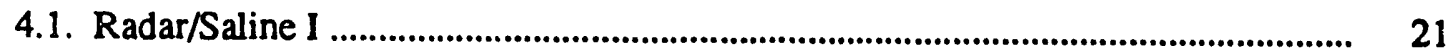

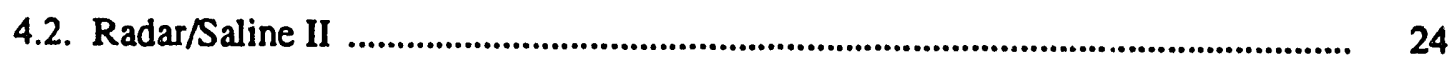

4.3. Neretnieks Tracer Tests ................................................................................... 31

5.0. MODELING THE FIRST RADAR-SALINE EXPERIMENT .................................. 41

5.1. C1-2 Results ................................................................................................ 41

5.2. Co-annealed Results ......................................................................................... 41

5.2.1. Co-annealed Configuration with a Background Permeability .................. 46

5.2.2. Co-annealed with Non-Zero Dispersivity .............................................. 46

5.3. Conclusions ................................................................................................... 46

6.0. MODELING THE SECOND RADAR-SALINE EXPERIMENT ................................ 49

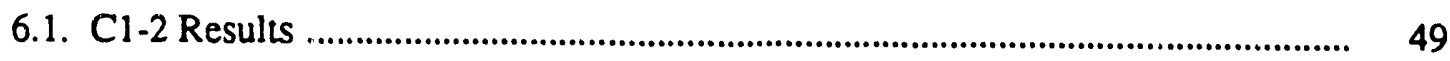

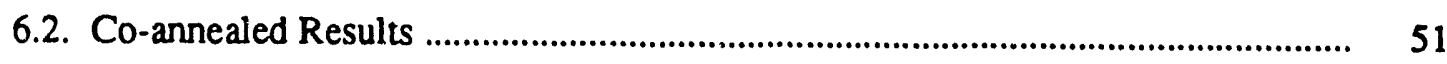

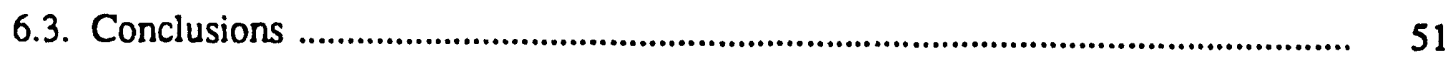




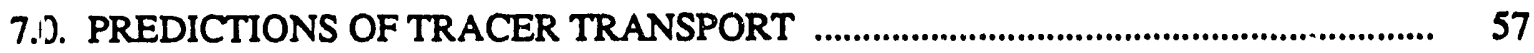

7.1. Predictions of Transport to the Drift .............................................................. 57

7.1.1. Case 1: Transport from T1 .......................................................................... 57

7.1.2. Case 2: Transport from $T 2$.................................................................. 57

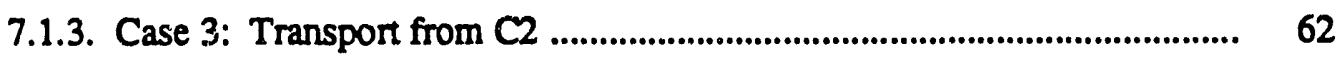

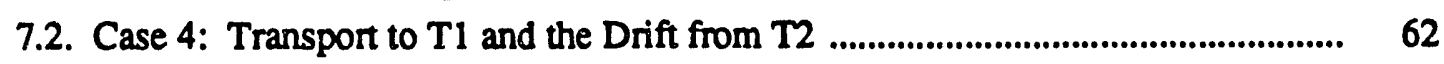

7.3. Conclusions ............................................................................................................ $\quad 62$

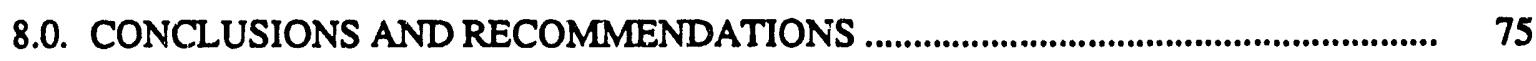

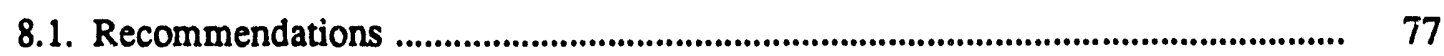

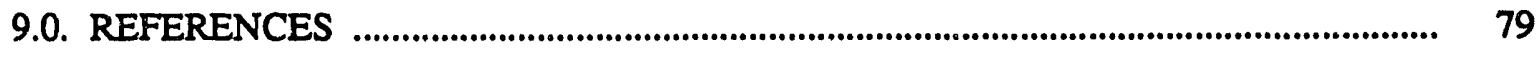




\section{List of Figures}

Page

Figure 1.1. Plan view of the SCV site. The heavy lines represent the traces of the seven major fracture zones in the SCV block.

Figure 2.1a. The central part of the mesh used for annealing the twodimensional model showing the well intersections. This is a subvertical plane looking towards the west.

Figure 2.ib. The five nested grid regions showing the locations of the intersections between the borehole intervals and the grid planes as well as the intersection with the Z-shaft.

Figure 2.2. The C1-2 Annealed configuration.

Figure 2.3. The co-annealed configuration.

Figure 4.1a. The geometry of the Radar/Saline Tracer Experiments (after Olsson, 1991b, p. 6). The Validation Drift shown in the figure was excavated after after Radar/Saline I. During Radar/Saline I, the D-holes lie within the boundaries of the drift shown on the figure. The drift formed the sink for Radar/Saline II.

Figure 4.1b. Relative location of the boreholes intersecting the H-zone. Saline tracer was injected in borehole $\mathrm{C} 2$ near the intersection with the $\mathrm{H}$-zone. The lines show where the tomogranhic planes intersect the H-zone (after Olsson 1991b, p. 62). The Z-shaft lies to the lower left off the page of this figure.

Figure 4.2a. Injection flow rate versus time for Radar/Saline I. The time refers to the start of saline tracer injection (after Olsson, 1991a, p. 23).

Figure 4.2b. Total flow entering the H-zone from boreholes D2 - D6 (after Olsson et al., 1991a, p. 49).

Figure 4.3. Head versus time in the injection interval in C2 (after Olsson et al., 1991a, p. 23).

Figure 4.4. Breakthrough of bromide in the total flow from D2-D6 for RSI (after Olsson et al., 1991a, p. 41). 
Figure 4.5. Composite of difference tomograms made approximately $290 \mathrm{hrs}$ after start of saline tracer injection for RSI. The injection point is shown by the long arrows. The dotted lines show the trace of the H-zone in the plane of the tomograms (after Olsson, 1991b, p. 142).

Figire 4.6. Conceptual model of saline tracer flow within the H-zone based on the radar difference tomograms. The boxes indicate when and where saline tracer is observed along the lines of intersection between zone $\mathrm{H}$ and the tomographic planes (after Olsson, 1991a, p. 99).

Figure 4.7. Injection flow rate versus time for Radar/Saline II. The time refers to the start of saline tracer injection (after Olsson, 1991b, p. 35).

Figure 4.8. Head versus time in the injection interval in C2 for Radar/Saline II (after Olsson, 1991b, p. 42).

Figure 4.9. Summarized breakthrough in the Validation Drift for RSII (after Olsson et al., 1991b, p. 48).

Figure 4.10. Breakthrough of saline tracer in borehole $\mathrm{T} 1$, sections 2 and 3 (after Olsson, 1991b, p. D14). (after Olsson, 1991b, p. D15).

Figure 4.12. A composite attenuation difference tomogram made approximately $290 \mathrm{~h}$ after tracer injection for Radar/Saline II (after Olsson, 1991b, p. 143).

Figure 4.13. Diagram of attenuation increase in zone $H$. The boxes indicate when and where saline tracer is observed along the lines of intersection between zone $\mathrm{H}$ and the tomographic planes (after Olsson, 1991b, p. 131).

Figure 5.1. Simulated tracer breakthrough at the D-holes for RSI plotted against the data for the simulation period of $400 \mathrm{hrs}$ calculated using the $\mathrm{C1}$-annealed configuration. Dots are data; the line is the simulation.

Figure 5.2. A snapshot of concentration in the C1-annealed configuration at approximately $270 \mathrm{hrs}$ for RSI. The wells are shown by black dots. Dark blue is high concentration and red is zero. This figure can be compared to Figure 4.6. 
Figure 5.3. Simulated tracer breakthrough at the D-holes for RSI plotted against the data for the simulation period of $300 \mathrm{hrs}$ calculated using the co-annealed configuration. Dots are data; the line is the simulation.

Figure 5.4. A snapshot of concentration in the co-annealed configuration at approximately $270 \mathrm{hrs}$ for RSI. The wells are shown by black dots. Dark blue is high concentration and red is zero. This figure can be compared to Figure 4.6. Note that the tracer concentration seen in the lower left is due to the sink formed by the $\mathrm{Z}$ shaft.

Figure 6.1. Simulated tracer breakthrough at the Validation Drift for RSII plotted against the data for the simulation period of $400 \mathrm{hrs}$ calculated using the $\mathrm{Cl}$-annraled configuration. Dots are data; the line is the simulation.

Figure 6.2. A snapshot of concentration in the C1-annealed configuration at approximately $347 \mathrm{hrs}$ for RSII. The wells are shown by black dots. Dark blue is high concentration and red is zero. This figure can be compared to Figure 4.13.

Figure 6.3. Simulated tracer breakthrough at the Validation Drif for RSII plotted against the data for the simulation period of $300 \mathrm{hrs}$ calculated using the co-annealed configuration. Dots are data; the line is the simulation.

Figure 6.4. A snapshot of concentration in the co-annealed configuration at approximately $347 \mathrm{hrs}$ for RSII. The wells are shown by black dots. $C 2$ is at the lower right; the Validation Drift in the center. Dark blue is high concentration and red is zero. This figure can be compared to Figure 4.13.

Figure 7.1. Predicted breakthrough into the Validation Drift from T1 for the C1-2 configuration. The data is shown by dots, and the simulation by the solid line.

Figure 7.2. Snapshot of concentration for Case 1, injection in $\mathrm{T} 1$ for the C1-2 configuration.

Figure 7.3. Predicted breakthrough into the Validation Drift from $\mathrm{Tl}$ for the 60 co-annealed configuration. The data is shown by dots, and the simulation by the solid line.

Figure 7.4 Snapshot of concentration for Case 1, injection in T1 for the coannealed configuration. 
Figure 7.5. Predicted breakthrough into the Validation Drift from $\mathrm{T} 2$ for the

C1-2 configuration. The data is shown by dots, and the simulation by the solid line.

Figure 7.6. Snapshot of concentration for Case 2, injection in $\mathrm{T} 2$ for the $\mathrm{C1}$ configuration.

Figure 7.7 Predicted breakthrough into the Validation Drift from $\mathrm{T} 2$ for the co-annealed configuration. The data is shown by dots, and the simulation by the solid line.

Figure 7.8. Snapshot of concentration for Case 2, injection in T2 for the coannealed configuration.

Figure 7.9. Snapshot of concentration for Case 3, injection in $\mathrm{C2}$ for the C1-2 configuration.

Figure 7.10. Predicted breakthrough into the Validation Drift from $\mathrm{C} 2$ for the co-annealed configuration. The data is shown by dots, and the simulation by the solid line.

Figure 7.11. Snapshot of concentration for Case 3, injection in C2 for the coannealed configuration.

Figure 7.12 Predicted breakthrough to $\mathrm{T} 1$ from $\mathrm{T} 2$ in the configuration C1-2. The data is shown by dots, and the simulation by the solid line.

Figure 7.13. Snapshot of concentration for Case 4, injection into $\mathrm{T} 2$ and collection in $\mathrm{T} 1$ and the drift for the $\mathrm{Cl}-2$ configuration.

Figure 7.14. Predicted breakthrough to $\mathrm{T} 1$ from $\mathrm{T} 2$ in the co-annealed configuration. The data is shown by dots, and the simulation by the solid line.

Figure 7.15 Snapshot of concentration for Case 4, injection into T2 and collection in $\mathrm{T} 1$ and the drift for the co-annealed configuration. 
- ix -

\section{List of Tables}

Page

Table 4.1. Conditions modeled to predict the Neretnieks' tracer tests. 39

Table 7.1. Estimates of first arrival and maximum concentration for the 74 tracer tests. 


\begin{abstract}
Tracer simulations for transport in a fracture zone were made based on two equivalent discontinuum models of a fracture zone (the H-zone) at the Stripa Mine. The two models were derived from simulated annealing inversions of hydraulic tests in the H-zone. The first inversion was based on a test from the $\mathrm{C} 1$ hole and the resulting configuration is called "C1-2." The second is based on simultaneous inversion of both the C1-2 test and the Simulated Drift Experiment (SDE) (Black et al., 1990) and the resulting configuration is called the "co-annealed" configuration. The C1-2 and the co-annealed configurations are derived in (Long et al. 1992). The first Radar/Saline (RSI) case simulated transport from one borehole to a collection of boreholes, the D-holes which all intersect the H-zone. A subsequent set of tests consisted of transport from points in the H-zone to the Validation Drift which had been excavated through the Dholes. The experimental breakthrough curve from RSI was used to calibrate the model by changing the ratio of flow to velocity in the conductive elements of the model to match the breakthrough curve. Only advective dispersion of tracer was allowed. This calibrated model was then used to predict the second saline tracer experiment (RSI). The source for RSII was the same as RSI, but the sink was the Validation Drift (VD). The calibrated model did not do too badly calculating arrival time but significantly over estimated the maximum $C / C_{0}$. Subsequently a series of tracer tests to the drift and to a borehole near the drift were simulated. Data from these tests have been compared to the simulations.
\end{abstract}

The simulated RSI breakthrough curve for the C1-2 configuration over-estimates dispersion and the co-annealed configuration underestimates it, implying that too many pathways are available in the C1-2 case and 000 few in the co-annealed case. This indicates that a series of configurations derived from repeat annealing of interference data may provide a reasonable estimate of advective dispersion. 
In the models, the change in boundary conditions from RSI to RSII was accompanied by an increase in the maximum $C / C_{0}$. However, in actuality the excavation left the maximum $C / C_{0}$ relatively unchanged. The increase in $C / C_{0}$ between the simulations of RSI and RSII occurs because the injected water is a greater proportion of the inflow to the drift than the inflow to the D-holes. The fact that the same trend is not observed in the data may be due to factors not included in the models, such as the effects of excavation and the resulting two-phase flow near the drift, the fact that the remaining D-holes were left open and could be pulling tracer away from the drift, or additional unaccounted for sinks.

Based on the simulations of the Neretnieks tracer tests, we expect to see cases where tracer does not show up at the drift at all. The simulations indicate that tracer that does show up will have rather low concentrations.

A comparison with the data shows that, in general the simulations are able to predict arrival time quite well. This means that the information from the interference tests was a good prediction of tracer travel path length. Prediction of the maximum $\mathrm{C} / \mathrm{C}_{0}$ was not as good but was fairly close in about half the predictions.

The most important recommendation of this report is that future studies of tracer transport in fracture networks should be done solely from boreholes which are held at pressures above atmospheric to maintain single phase conditions There are many things that are not fully understood about the physics of the transport phenomenon in fractures and coupling these two problems makes it very difficult to interpret the experiments. The SCV project has shown clearly that the hydrology of excavations is not well understood. 


\section{Acknowledgements}

This work was supported by the Office of Strategic Planning and Internal Programs within the Office of Civilian Radioactive Waste Management of the U. S. Department of Energy under Contract DE-ACO3-76SF00098. We are grateful for their support. We also wish to thank the

Stripa Project for accommodating and supporting our efforts. We thank Amy Davey Mauldon and Christine Doughty for reviewing this manuscript. 


\subsection{INTRODUCTION}

This report describes a series of numerical simulations of tracer tests that were performed in a fracture zone (the H-zone) at the Stripa Mine in Sweden. The tracer simulations are based on Equivalent Discontinuum models which were developed based on geophysical measurements and hydraulic interference data (Long et al., 1992). The transport simulations are calibrated to one set of saline tracer breakthrough curves (from the first radar/saline experiment, RSI) and these calibrated models are used to predict another series of breakthrough curves. Predicted breakthrough curves can be compared to the actual data and simulated "snapshots" of concentration in the plane of the fracture zone can be compared to radar difference tomograms made during the saline tracer experiments.

\subsection{Background}

Investigations related to the geologic storage of nuclear waste have been ongoing at the Stripa Mine in Sweden for more than ten years. The latest of these investigations is called Phase III and is sponsored by OECD Nuclear Energy Association (NEA) as an international cooperative effort managed by the Swedish Nuclear Fuel and Waste Management Company (SKB).

The Stripa Phase III project includes the Site Characterization and Validation (SCV) experiment, which is designed to test current abilities to characterize fractured rock before it is used for nuclear waste storage. The effort is centered on a block of rock $230 \mathrm{~m}$ long $\times 200 \mathrm{~m}$ wide $\times 190$ $\mathrm{m}$ thick centered at a depth of about $330 \mathrm{~m}$. The block lies to the west of a previous experimental site, the 3-D Migration site (Figure 1.1).

A series of predictions of flow and drawdown were made for the SCV block and compared to measurements. These are documented in Long et al. (1992). This report presents a series of tracer transport simulations based on the flow models given in Long et al. (1992). The simulations are compared to a series of transport experiments performed in the SCV block. 


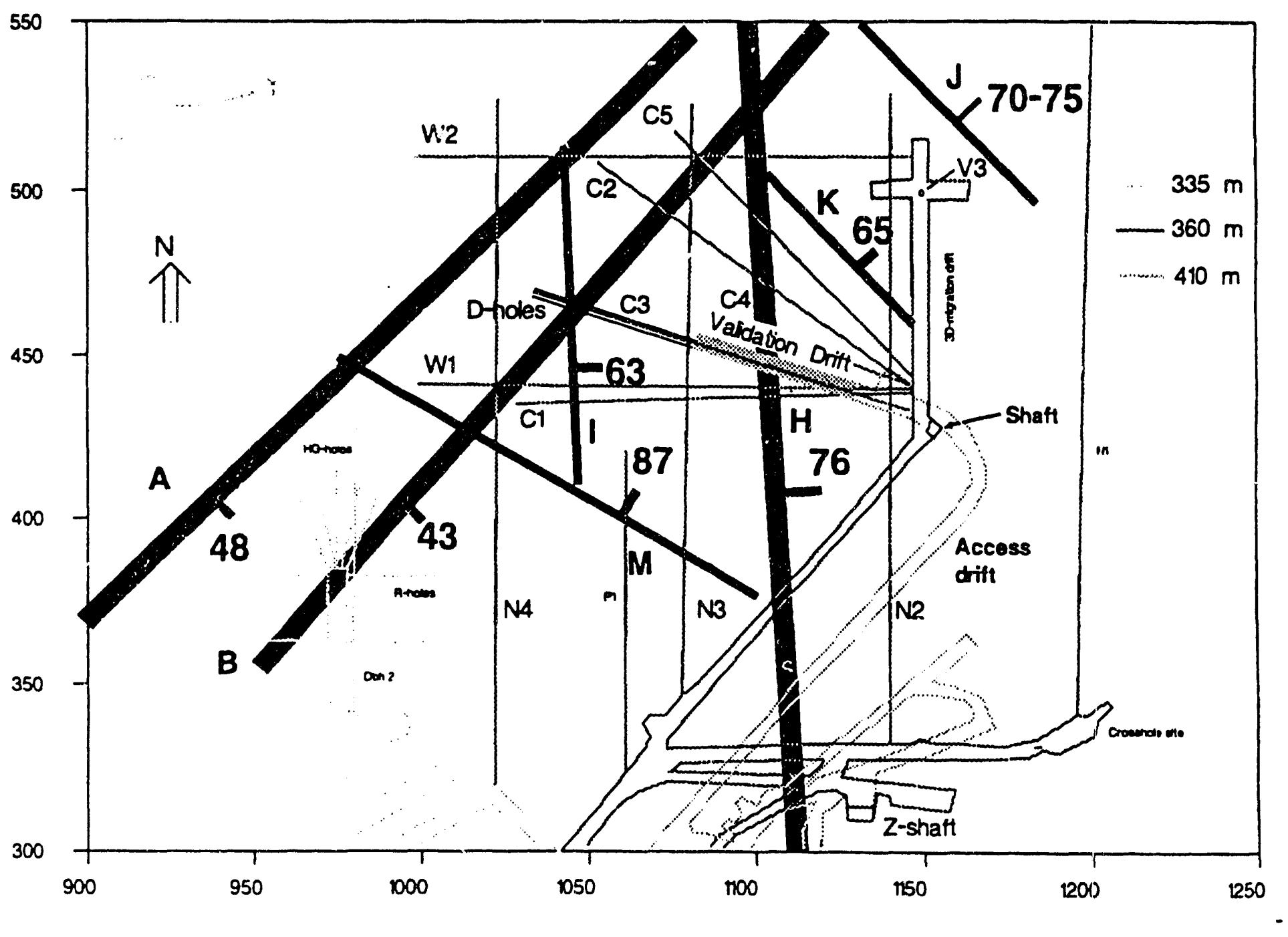

XBL $921-5201$

Figure 1.1. Plan view of the SCV site. The heavy lines represent the traces of the seven major fracture zones in the SCV block. 


\subsection{Modeling Approach}

The tracer simulations described in this report are based on hydrologic models of a fracture zone (the H-zone) that were described in Long et al. (1992). These models can be characterized as "Equivalent Discontinuum" models (EQDM). An Equivalent Discontinuum model is a model which uses a partially filled lattice of one-dimensional conductors to represent equivalent fracture flow paths. The model is designed to represent the discontinuous nature of fracture flow in the simplest manner.

At the Stripa mine, the granitic rock mass is ubiquitously fractured. However, evidence at Stripa points to hydrology which is dominated by fracture zones (Olsson et al., 1991; Black et al., 1991). Consequently, for the SCV block, equivalent discontinuum models have been created based on the assumption that nearly all of the hydrology is controlled by fracture zones. The fracture zones have been identified and located through extensive characterization efforts (Olsson et al., 1991 and Black et al., 1991).

Three-dimensional flow modeis were created by Long et al., 1992 as partially filled twodimensional lattices of one-dimensional conductors which lie on planes corresponding to the fracture zones given by Black et al. (1991). In addition, two-dimensional flow models were created as lattices of one-dimensional conductors lying on a plane corresponding to the H-zone which is the major hydrologic feature in the SCV block. A hydrologic inversion technique called "Simulated Annealing" (Long et al., 1992; Davey et al., 1989) was used to find configurations of these lattices that reproduced a series of hydrologic interference tests conducted in the fracture zones.

A series of tracer tests were conducted in the H-zone. These are referred to as Radar/Saline I (RSI), Radar/Saline II (RSII) (Olsson et an., 1991a,b), and the Neretnieks tracer studies. In RSI a saline tracer was injected into the hole $C 2$, near the intersection of $C 2$ and the H-zone. A sink was created above the source in the H-zone by lowering the pressure in six parallel boreholes (the Dholes) in the intervals intersected by the H-zone. The D-holes were drilled in an array designed to simulate the hydraulic conditions imposed by a two-meter diameter drift. RSII was conducted in a similar manner to RSI, but after the Validation Drift (VD) was excavated through the D-holes. 
In both RSI and RSII, radar tomography measurements were made before and after the injection of saline fluid in a series of planes that intersect the H-zone. The Neretnieks tracer studies were also conducted after the excavation of the Validation Drift. In these studies a series of different tracers were injected into the H-zone at a variety of locations and the arrival of the tracer was monitored at the drift. In addition, one tracer test was conducted by injecting tracer into the hole T2 at the H-zone while the hole T1 was opened to atmospheric pressure. Tracer arrival was monitored at the drift and at $\mathrm{T} 1$.

The models created for flow predictions do not contain all the parameters necessary to simulate tracer transport experiments. Simulated Annealing is used to match interference tests. Suitable matches were found with lattice elements that all have the same conductance. The pattem of elements accounts for the observed distribution of head and the magnitude of the conductance of the elements accounts for the magnitude of the flow and drawdowns. Additional parameters must be specified in each element in order to simulate the concentration distribution of an injected tracer in space and time. At a minimum, the ratio of flow to velocity $(q / V)$ in each element, i.e., an equivalent cross-sectional area $\left(A_{E}\right)$ must be specified. The same value of $A_{E}$ was used for every element. If the transport is advection dominated, then this $\mathrm{g} / \mathrm{V}$ is all that is needed. However, if there is a significant amount of dispersion within the individual fracture flow elements, then an equivalent element dispersion coefficient must be specified as well. Flow tests do not provide any information for determinin $n_{b}$ these parameters.

The following approach was followed to simulate the tracer tests. First, RSI was simulated and used to find appropriate values of $A_{E}$ and element dispersion coefficient. These values were then used to simulate the RSII test. Then the series of Neremieks tracer tests were simulated with these values. In all cases, breakthrough curves and snapshots of concentration in the lattice elements are generated with the simulation results. For the Radar/Saline cases, the snapshots can be compared to the radar tomography results at similar times. 


\subsection{Limitations of this Study}

There are four major limitations that concem these simulations. The first is that all the tracer simulations were based on a limited number of inversions of the hydraulic interference data. One of the important principles which should be applied in using Simulated Annealing is that the inversion is non-unique and one should produce a series of inversions and use these as a statistical sample for making predictions. However, limitations of data, time and budget allowed only two cases to be studied here. Consequently, this work should be looked at as preliminary in terms of prediction and useful as an example.

Second, the form of Simulated Annealing applied to the SCV site resulted in lattices with elements having the same conductance. This model is sufficient to match the hydraulic interference data, but the same data could also be matched with a distribution of lattice conductors in which case a significant amount of macro-dispersion can be achieved. A better approach would have been to match RSI by using Simulated Annealing to find conductance distributions that match the breakthrough data. However, this technique was beyond the scope of this project.

Third, the excavation of the Validation Drift caused enormous changes in the flow field around the drift. These are very poorly understood (Long et al., 1992). There is evidence that a major contributor to the modification of flow near the drift is the de-gassing of the water as it drops from pressure heads of about $200 \mathrm{~m}$ to zero. The effects of two-phase flow on transport in fractures is not well understood.

Fourth, it should be remembered that the models are no better than the data and that errors related to poorly controlled test conditions have not been completely evaluated. In particular, the tracer tests were simulated as if they were carried out under steady flow conditions that were not always achieved in reality. Also, any errors incurred in the flow modeling were simply transferred to these tracer calculations.

It is a research project in itself to determine how best to predict transport in fractured media and this research was not within the scope of the Stripa Project. The major contribution of this work is simply to make the predictions and leam something about the use of EQDM in tracer 
transport simulations.

\subsection{Structure of this Report}

Section 2 of this report presents the flow models defined in Long it al. (1992). Section 3 describes the modeling approach used for tracer transport simulation. Section 4 summarizes all the tracer transport data that was available for analysis. Sections 5 and 6 present the simulations of RSI and RSII respectively. The Neretnieks tracer simulations are given in Section 7. Section 8 draws some conclusions about what can be learned by these studies including some suggestions for further transport experiments. 


\subsection{TWO-DIMENSIONAL MODELS USED FOR TRANSPORT CALCULATIONS}

Long et al. (1992) present a series of three- and two-dimensional Equivalent Discontinuum models used for predicting flow and drawdown in the SCV block. These models are based on the conceptual model for fracture zones given in Black et al. (1991). The Simulated Annealing inversions applied to these models were based on two hydraulic experiments: the C1-2 interference test and the Simulated Drift Experiment (SDE) as documented in Black et al. (1991).

The three-dimensional models were considered of marginal utility in simulating the transport experiments. The three-dimensional models were lf, detailed than the two-dimensional models in the vicinity of the H-zone which was where all the tracer tests were conducted. None of the other fracture zones were expected to play a significant role in these tracer tests. This was confirmed by the radar/saline results, which show that the majority of tracer transport is in the Hzone. Some amount of tracer was seen in a previously undetected feature, the S-zone (Olsson et al., 1991a,b). The S-zone could have been included in the transport model, but there was very little information available on the geometry and properties of this zone and so it was neglected.

In the two-dimensional models, the boreholes intersect the H-zone essentially only at a point. In reality, the $\mathrm{H}$-zone is some $\mathbf{2 0}$ meters thick and the boreholes intersected the $\mathrm{H}$-zone over a finite interval. In most of the boreholes, the H-zone was contained in one packer interval which means that the data obtained from the interval was consistent with a two-dimensional model. The T-holes, however, were packed off in three intervals over the intersection with the H-zone. This allowed for injection and monitoring in these intervals simultaneously. The twodimensional model represents the sum of the behavior of these three intervals.

Only two two-dimensional inversions were available. Both of these inversions were based 
on the latice template shown in Figure 2.1. The first was a model annealed to the C1-2 test alone. The second was a model co-annealed to both the C1-2 test and the steady-state results of the SDE. As reliable predictions made with this type of model require that Monte-Carlo type studies be done by repeating the stochastic inversion a number of times. This should be kept in mind while reviewing the results below.

\subsection{C1-2 Annealed Case}

The lattice configuration shown in Figure 2.2 was annealed to the $\mathbf{C 1 - 2}$ interference test. The C1-2 test was conducted hy pumping from the interval number 2 in $\mathrm{Cl}$ which intersects the H-zone and monitoring the transient drawdowns in the H-zone at W1, W2, C2, C4 and C5. The C1-2 annealing was able to match all of the transient drawdown curves quite well and this configuration was used to predict the flow into the D-holes. The predicted flow was the same as the measured flow to two significant figures $(0.771 / \mathrm{min})$.

Only one of the D-holes (D1) is labeled on the figure. The array of D-holes was actually modeled as five nodes lying on a cross. Dashed iines on the figure represent lattice elernents that are present but are deadends or not connected. Only the central part of the configuration is shown. The Z-shaft, which provided an hydraulic sink in the H-zone is located to the lower left of the grid lattice and is not seen on this plot. It is clear from the network shown that several distinct pathways exist between the radar/saline source interval in C2 and the sink at the D-holes.

\subsection{Co-Annealed Case}

The lattice configuration shown in Figure 2.3 was simultaneously annealed to both the C1-2 test and the steady state results of the Simulated Drift Experiment (SDE). The SDE was carried out by holding the D-holes at a constant low pressure and measuring the inflow to the holes as well as the drawdown in the surrounding borehole intervals. The steady-state response was estimated by Black and Perry (personal communication) based on the transient data. Coannealing was set up to find configurations that matched the steady-state SDE drawdowns and inflow as well as the transient drawdowns from the C1-2 test. This configuration was judged to 


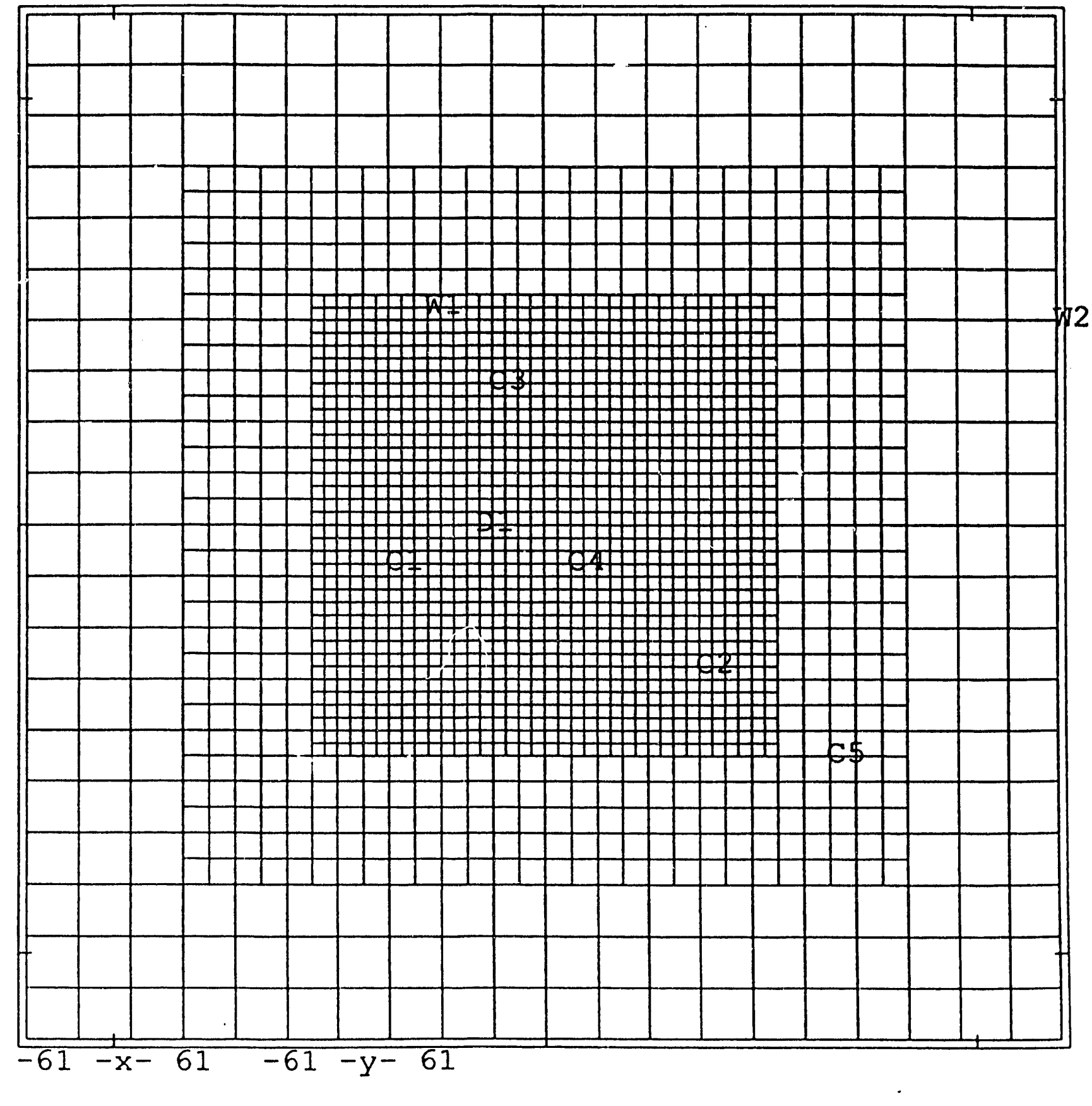

XBL $921-5202$

Figure 2.1a. The central part of the mesh used for annealing the two-dimensional model showing the well intersections. This is a subvertical plane looking towards the west. 


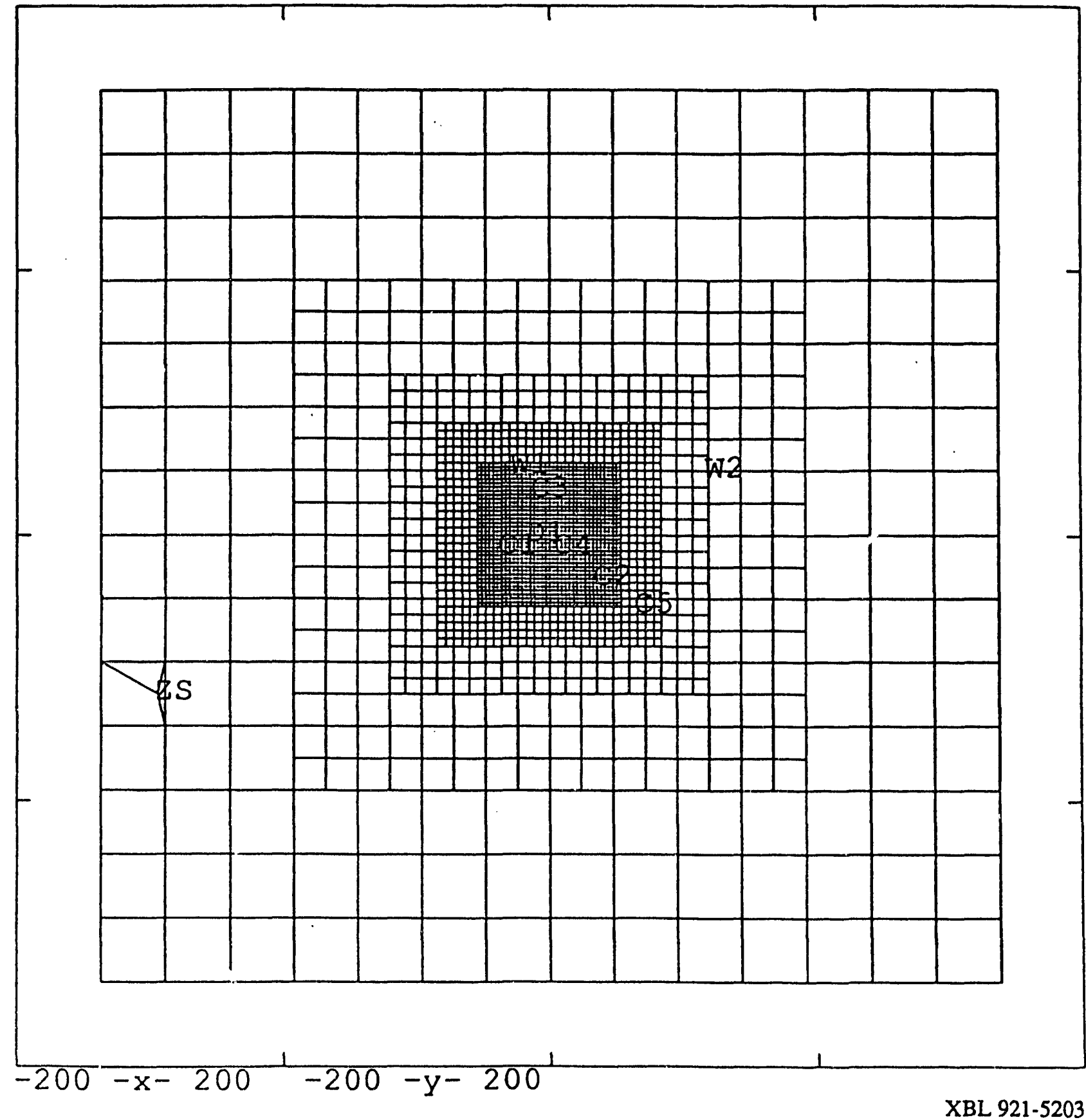

Figure 2.1b. The five nested regions showing the location of the intersections between the borehole intervals and the grid planes as well as the intersections with the $\mathrm{Z}$-shaft. 


\section{2-D C1-2 annealed mesh (Dead-end elements dotted)}

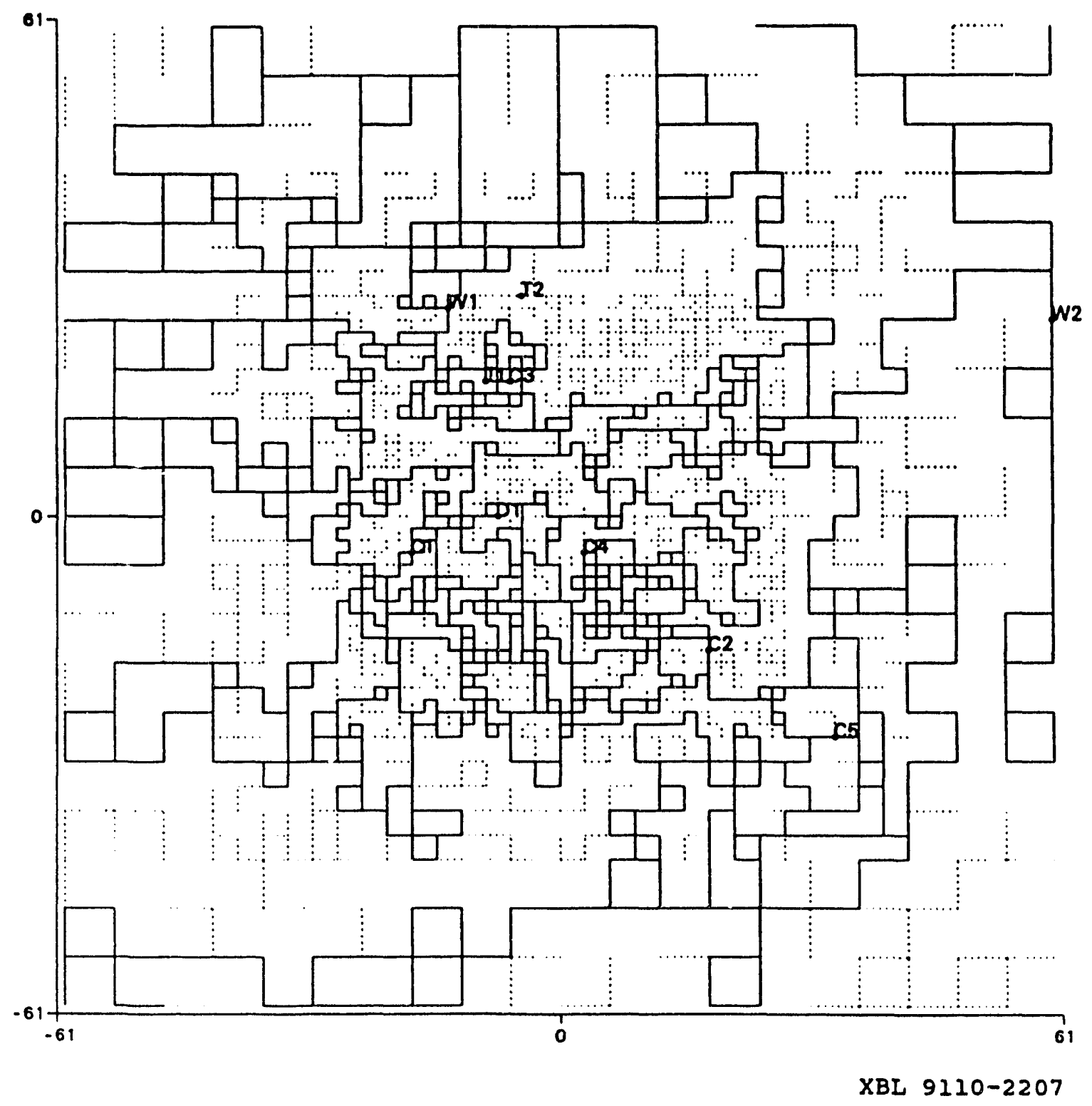

Figure 2.2. The C1-2 annealed configuration. 


\section{2-D Mesh co-annealed to C1-2 and SDE (Dead-end elements dotted)}

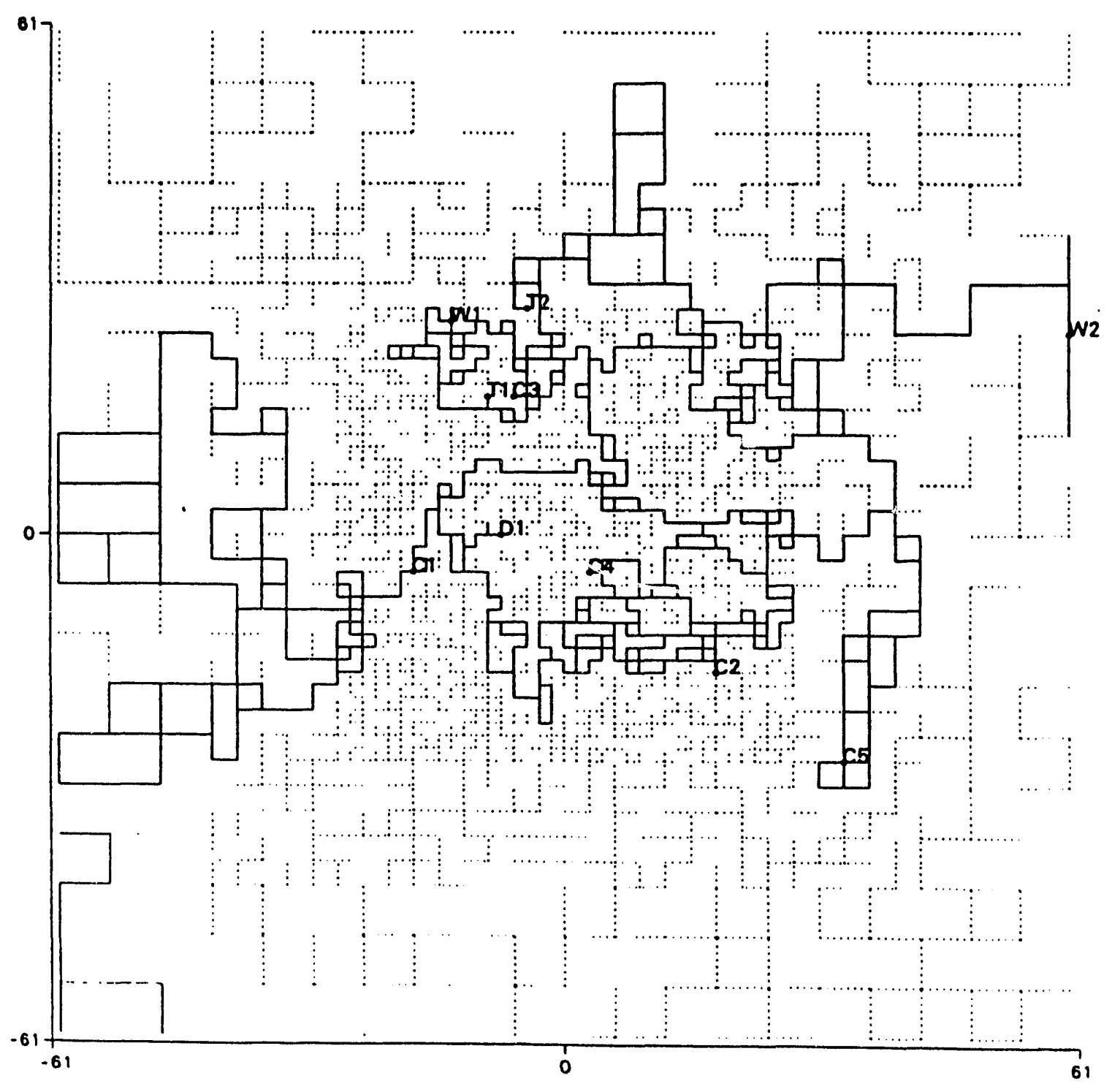

XBL 9110-2205

Figure 2.3. The co-annealed configuration. 
include more hydraulic information than the C1-2 case and was used to predict the response of the V'alidation Drift.

There is a marked difference between the configuration annealed to C1-2 (Figure 2.2) and the co-annealed configuration (Figure 2.3). The co-annealed configuration is much sparser than the C1-2 case. Tracer going from $C 2$ to the D-holes can travel on more different pathways. Clearly the tracer response in the two networks will be quite different.

\subsection{Altering the Models to Represent the Excavation of the Validation Drift}

Long et al. (1992) give the details of the simulation of the Validation Drift using the coannealed model. Because the D-holes provided an extremely good approximation of the hydraulic boundary conditions imposed by a drift, the only modification needed for modeling the drift instead of the D-holes was to account for changes in hydraulic properties near the drift due to exiavation. A simple, heuristic approach was taken: a low-permeability skin was imposed on elements within 5 meters of the drift sufficient to reproduce the observed decrease in the flow. This value of skin is used to model both the RSII and the Neretnieks tracer tests. 


\subsection{SIMULATION OF TRACER TRANSPORT}

The three components of modeling the tracer tests are the numerical code, TRINET; the parameters needed by the code; and the choice of flow conditions. These are discussed below.

\subsection{TRINET}

TPMTET is an advection-dispersion code designed to calculate tracer transport on any twoor three-dimensional network of one-dimensional conductors (Karasaki 1986, 1987). The code incorporates a Lagrangian/Eulerian scheme with adaptive gridding which allows calculations that are virtually free of numerical dispersion, even with large Peclet numbers. The code is designed to simulate field tracer tests such as drift and pumpback tests or two-well circulations tests.

There are two mechanisms for dispersion within a fracture system. One is dispersion due to the network geometry itself. The other is dispersion and diffusion within individual fractures. TRINET models both effects by treating the geometry of the network explicitly and allowing for dispersion and diffusion within each fracture element. The model avoids numerical dispersion by creating additional grid points at the front.

The code first solves the flow field using a simple Galerkin finite-element method. The flow can be either steady state of transient, in which case the time derivative is treated in the usual finite difference manner. From the pressure distribution at a given time, the velocity distribution in the fracture network is calculated. The velocity is assumed to be uniform within a given onedimensional element.

The advection-dispersion equation for mass concentration is then solved by treating the equation in two stages. In this way it is possible to treat the advection term independently of the diffusion term and minimize the numerical dispersion. First the advection equation is solved by using the method of characteristics. The concentration profile at the end of each time step is, in effect, the initial-value distribution for a new advection problem. This profile is advected 
explicitly in the Lagrangan manner according to the velocity in each element. The advected front is then placed on the Eulerian grid, and new nodal points are generated to preserve the exact shape of the front. At every time step the element catalog is revised and the nodal points renumbered to keep the bandwidth minimized. The method of single-step backward particle tracking or reverse streak lines is used to obtain the concentration values for the fixed nodes of the fracture network. This step can cause a minor amount of numerical dispersion. The new concentration profile at the end of the advection stage is now the initial value for the dispersion calculation in the second stage. The dispersion is treated in the usual finite-element manner.

\subsection{Parameters Needed to Model Transport}

The Equivalent Discontinuum models that are the basis of the tracer calculations consist of a partially filled lattice of conductors. Each lattice element is specified by a length and a conductivity. The conductivity is proportional to the element spacing such that each part of the nested mesh has the same overall conductance. These models require further parameterization to model tracer transport.

The first parameter is the ratio of flux to velocity in each element, called the effective cross-sectional area, $\left(A_{E}\right)$. The flow model calculates the flow rate from one end of the element to the other and the ratio of flux to velocity is then used to determine how long it takes a particle to move the same distance. If the model is of sufficient detail, advective transport through multiple paths will disperse the tracer.

In addition, an equivalent element dispersivity can be used to describe spreading of the tracer within the elements. This dispersivity allows particles to essentially change flow tubes thin an element. The EQDM can be viewed as a collection of equivalent conductors each of which represents a collection of actual fractures in a flow path. In this light, the dispersivity represents the different possible travel times along this collection of fractures.

It is possible to create an EQDM with lattice elements of varying conductance. Such a model will be inherently more dispersive than a lattice with equal conductance elements. Along 
the same lines, the more finely discretized the lattice is, the more the model can capture advective dispersion. Current thinking about transport in heterogeneous materials is that it is much more promising to try to represent more details in the pathways than it is to represent the dispersive process with a large value of dispersivity. Comparison of tracer predictions for different levels of discretization or varying conductance lattices was beyond the scope of this report but is cleariy an important area of research.

One final point is that the lattice elements were designed to lie on a nested grid in order to save computer time. This means that it is not possible to represent the same level of advective mixing in the outer regions of the lattice as it is in the center. It was not necessary to correct for this problem because nearly all the transport of interest takes place in the central portion of the lattice.

The procedure for finding appropriate values of the ratio of $A_{E}$ and dispersivity was as follows. The first radar/saline test was simulated with a unit value of $A_{E}$ and zero dispersivity. Then the time scale of the simulated breakthrough curve is multiplied by a factor which gives the best fit to the actual breakthrough data. This factor is the best fit for $A_{E}$. If this fit is unsatisfactory, increasing values of dispersivity can be input to the model which must then be re-run to obtain the breakthrough curve. Re-running the simulation for every trial value of dispersivity is time consuming, making determination of dispersivity values difficult.

As will be seen below, it was possible to fit the breakthrough data using this procedure, however, the values of dispersivity that fit the data were large enough to diffuse the tracer throughout the H-zone. These results were unreasonable because the radar data do not support such smearing. Consequently, all the predictions were made on the basis of values of $A_{E}$ that gave a match to the breakthrough curve of RSI with zero dispersivity in the elements. In this way it is possible to examine how much of the advective transport process can be simulated by the EQDM derived from annealing pressure data. 


\subsection{Flow Conditions}

In all the tracer transport calculations given in this report, the flow system is assumed to be at steady-state. This assumption was not always very good as can be seen in Chapter 4 . Injection flow rates changed during both radar/saline tests, and it is very unlikely that steady flow took place during the Neretnieks tests because injections rates changed as each new tracer was injected. However, a large number of unknown parameters would be needed to model transport under transient conditions. Consequently steady conditions were assumed based on the best estimate of average flow.

In each case, constant head or no-fiow boundaries were applied to the outer edges of the models as explained in Long et al. (1992). For the inner boundaries (the D-holes or later the drift) constant flow boundaries were set to the value of flow that was measured in situ. However, for the case when the $\mathrm{T} 1$ hole was opened, the flow rates to the $\mathrm{T} 1$ hole and the drift were unavailable. In these cases, we used estimates based on the calculations given in Long et al. (1992). These estimates tumed out to be significantly in error, so the tracer predictions for this case are not very useful. The Z-shaft was included in all the models as a sink held at constant head as described in Long et al. (1992).

Finally it was necessary to pick the injection rates for the tracer. For RS I, the flow rate averaged over the first $300 \mathrm{hrs}$ was used. For RS II, the constant flow rate that was held for the first 200 hrs was used and transport was only simulated for this same period. After this point, the flow rate changed radically and steady flow was considered to be a bad assumption. For the Neretnieks tracer tests, tracer was simultaneously injected into more than one interval. All the injections that were going on during a specific test were modeled, but only one tracer was included in each simulation. That is, when modeling transport from one hole, tracer was injected into that hole and pure water into the other intervals that were being used for sources simultaneously. When these intervals are in the same borehole (T1 or T2), the total injection rate from that borehole was used and the concentration was reduced by an amount proportional to the relative injection rates. That is, if tracer of concentration, $C_{0}$, is injected at $11 /$ min into T1-1 and a dif- 
ferent tracer is injected into T1-2 at $21 / \mathrm{min}$, the T1-1 test is simulated by injecting tracer at 3 $1 /$ min with concentration equal to $C_{d} / 3$. 


\subsection{EXPERIMENTAL DATA}

Three sets of experiments were simulated. These were RSI, RSI and the tracer experiments carried out by Neretnieks et al. (personal communication). The Neretnieks experiments were carried out under two different sets of boundary conditions. These experiments are described below. The relevant results of the radar/saline tests are summarized here and the results of the Neretnieks experiments are shown with the simulations of Chapter 7.

\subsection{Radar/Saline I}

Figure 4.1 shows the generalized geometry of the Radar/Saline Tracer Experiment. Saline tracer was injected in borehole $\mathrm{C} 2$ where it intersects zone $\mathrm{H}$, a N-S striking zone dipping steeply to the east. A sink was created by keeping pressure low in the D-holes. In Figure 4.1, the Validation Drift is shown where the D-holes were during RSI. Radar tomography was done in the W1$\mathrm{C} 5, \mathrm{C} 5-\mathrm{C} 1$, and W1-C1 planes before, during, and after saline injection. Therefore, information about tracer travel in the $\mathbf{H}$-zone is only available along the lines of intersection between the tomography planes and the H-zone.

$A C_{0}=2 \%$ saline solution was injected in $C 2$ at an average rate of $217 \mathrm{ml} / \mathrm{min}$ for 360 hours while the pressure head in the D-holes was kept at $165 \mathrm{~m}$ (datum was at the $360 \mathrm{~m}$ level in the mine). In a second phase of this experiment, the pressure head in the D-holes was lowered to atmospheric pressure to simulate the drift. This second phase was not simulated because the change in boundary conditions led to a transient flow field superimposed on the transport problem. Tracer concentration in the D-holes was monitored continuously. The total flow rate into the D-holes during the first phase of this experiment was observed to be $0.34 \mathrm{l} / \mathrm{min}$.

Figure 4.2a shows the injection flow rate versus time for RSI. Time zero refers to the start of tracer injection. Perturbations in the flow field may have been caused by the injection spikes. 


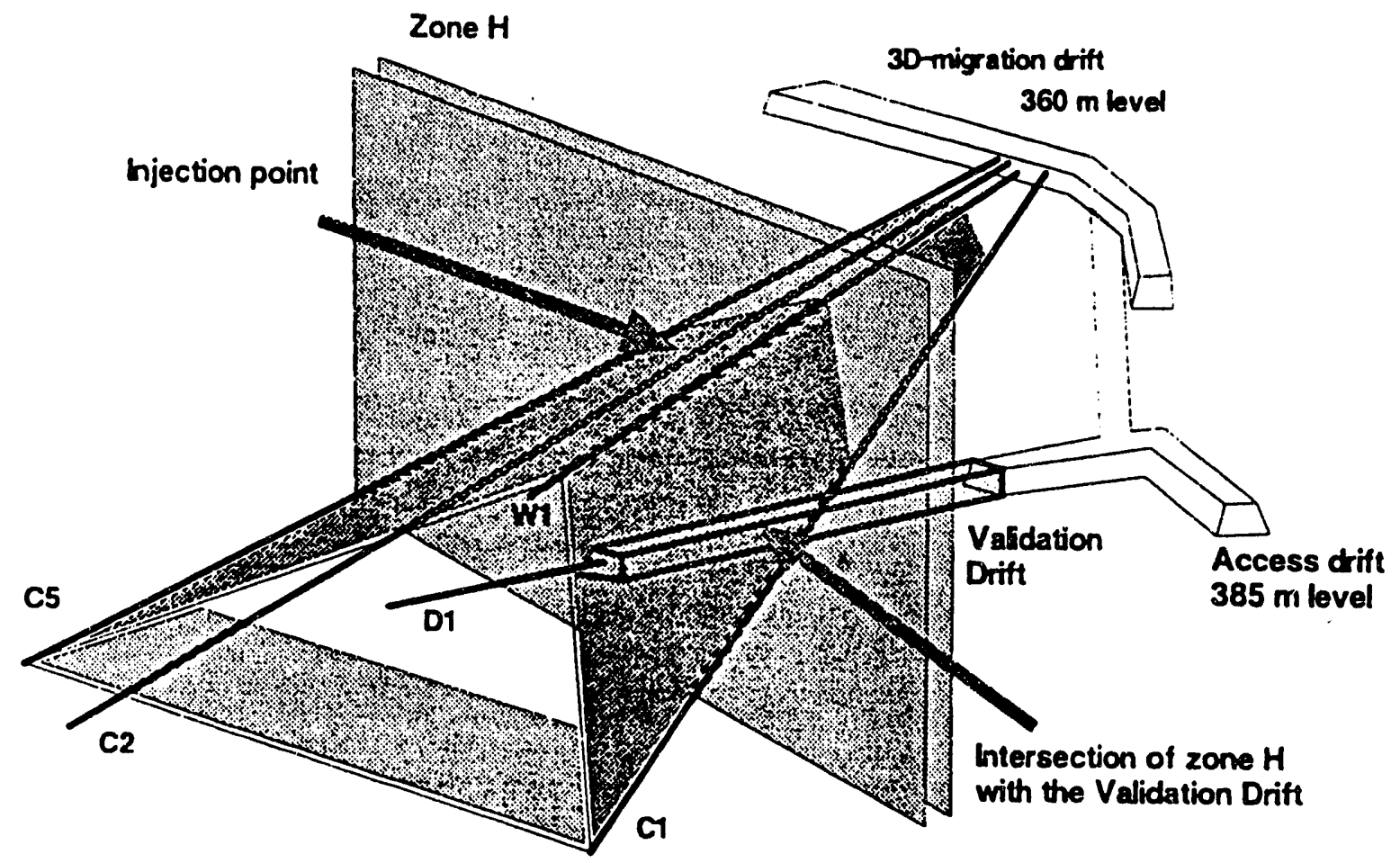

Tomographic planes

XBL 921-5204

Figure 4.1a. The geometry of the Radar/Saline Tracer Experiments (after Olsson, 1991b, p. 6).

The Validation Drift shown in the figure was excavated after Radar/Saline I. During Radar/Saline I, the D-holes lie within the boundaries of the drift shown on the figure. The drift formed the sink for Radar/Saline II. 


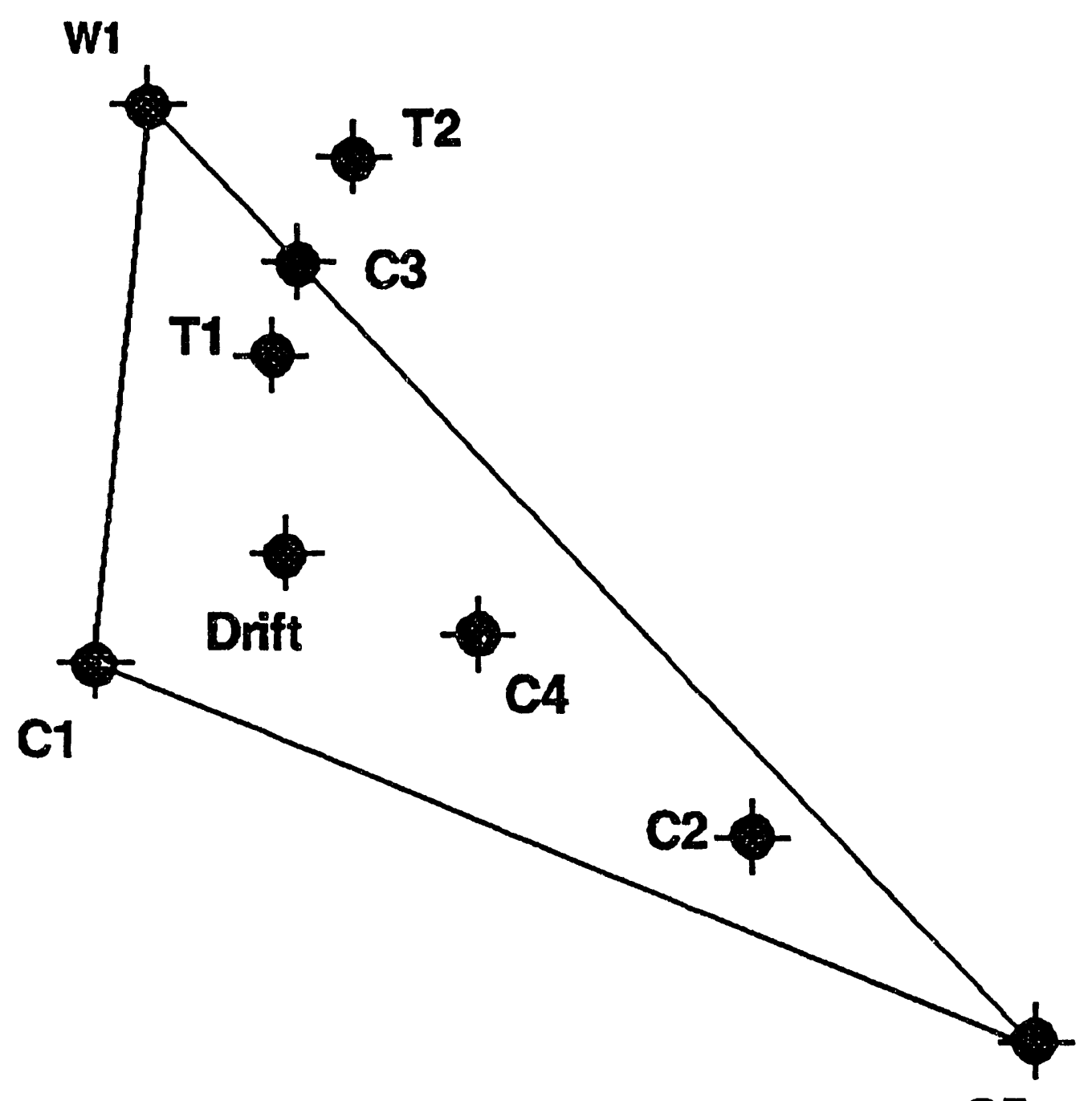

C5

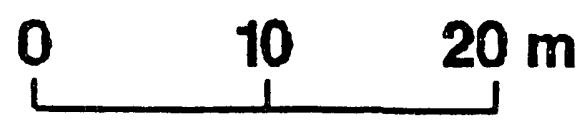

XBL 921-5205

Figure 4.1b. Relative location of the boreholes intersecting the H-zone. Saline tracer was injected in borehole $\mathrm{C} 2$ near the intersection with the $\mathrm{H}$-zone. The lines show where the tomographic planes intersect the H-zone (after Olsson 1991b, p. 62). The Z-shaft lies to the lower left off the page of this figure. 
These were not modeled explicitly because steady flow conditions as explained in Section 3.0 were used. Figure $4.2 \mathrm{~b}$ shows the total flow entering boreholes D2-D6 from the H-zone.

Figure 4.3 shows the head versus time in the injection interval. The head at the injection interval is climbing during the entire first phase of RSI. Consequently, the assumption of steady flow conditions incurs some error.

Figure 4.4 shows the breakthrough curve for Bromide in the total flow into the intervals of D2-D6 (D1 was closed) which intersect the H-zone. During the first phase, the total tracer concentration did not plateau at the $\mathrm{D}$-holes. The highest concentration ratio, $\mathrm{C} / \mathrm{C}_{0}$, observed before the flow conditions were changed was about 0.33 . This means that the final $C / C_{0}$ would have been greater than 0.33 , but it is not known how much higher. There is an inflection in the first part of the arrival curve which may be indicative of the existence of more than one distinct flow path between $\mathrm{C} 2$ and the $\mathrm{D}$-holes.

Figure 4.5 shows a composite attenuation difference tomogram for the three planes at approximately 290 hours after injection of tracer began. The borehole C2 is projected on to the tomographic planes in order to show the injection point. The intersection of the H-zone and the tomographic planes is shown by a dotted line. Note that the C1-W1 plane is shown twice.

Figure 4.6 shows a summary of tracer flow along the intersection between the H-zone and the tomographic planes based on the difference tomography. Two possible flow paths outside of the H-zone are shown as zones $S$ and $T$. Neither of these correspond to any feature previously identified. Simulated snapshots of concentration in the H-zone can be compared to this summary plot.

\subsection{Radar/Saline II}

The generalized geometry of the second Radar/Saline Tracer Experiment was the same as that shown in Figure 4.1. Saline tracer was injected in borehole $\mathrm{C} 2$ where it intersects zone $\mathrm{H}$. The sink was created by the Validation Drift. As, in RSI, radar tomography was done in the W1$\mathrm{C} 5, \mathrm{C} 5-\mathrm{Cl}$, and $\mathrm{W} 1-\mathrm{Cl}$ planes before, during, and after saline injection. 


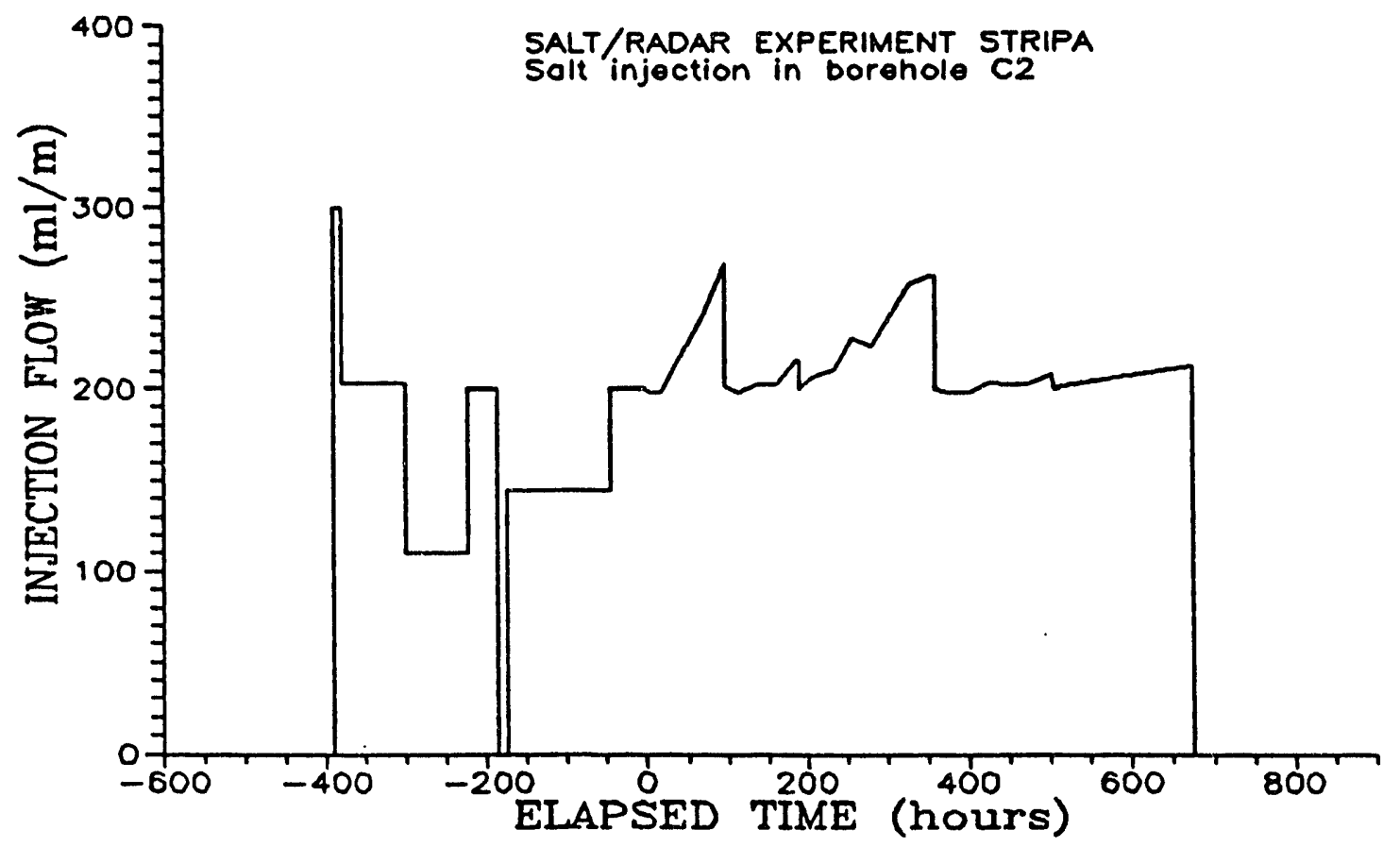

XBL $921-5206$

Figure 4.2a. Injection flow rate versus time for Radar/Saline I. The time refers to the start of saline tracer injection (after Olsson, 1991a, p. 23). 


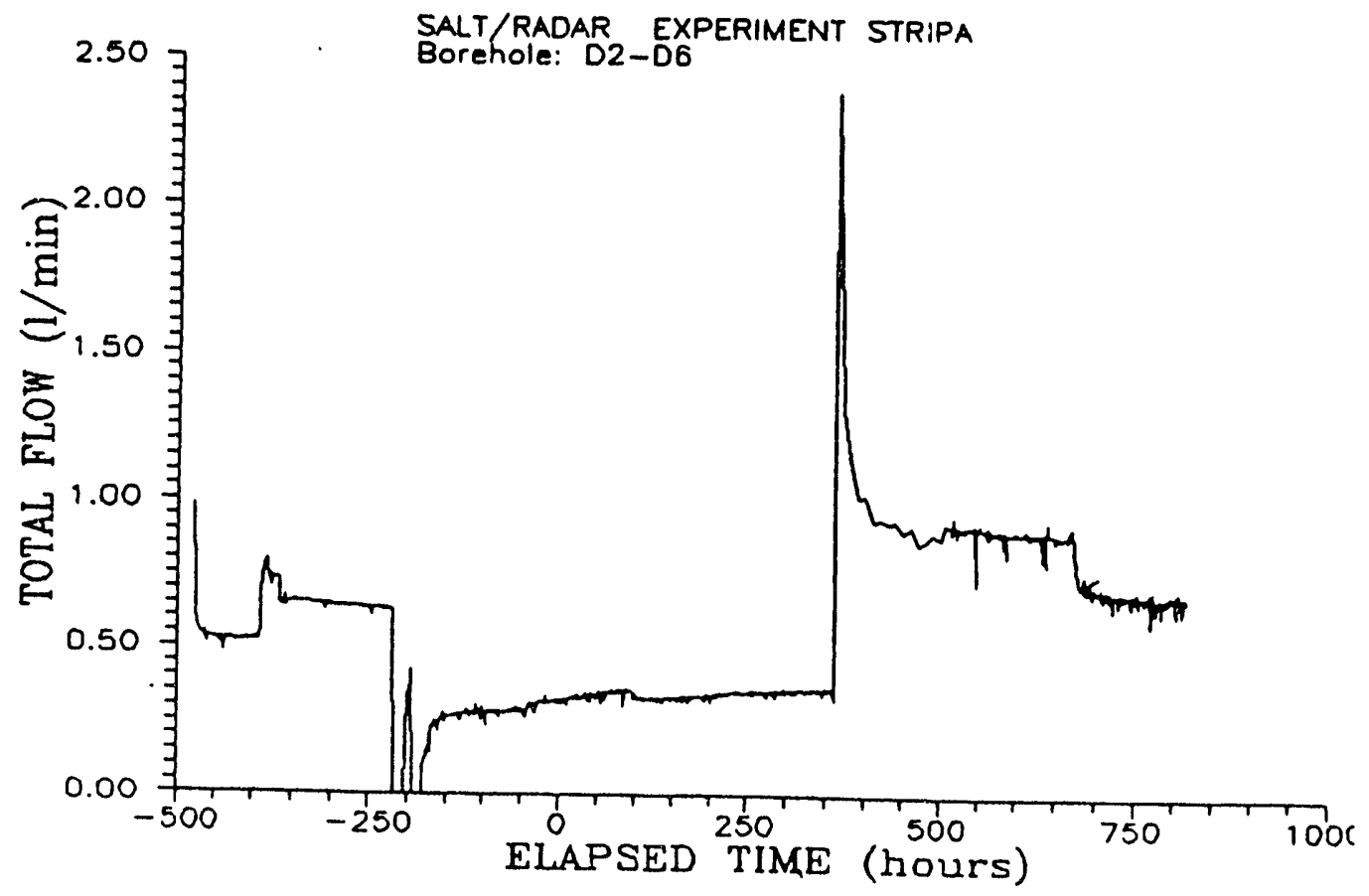

XBL 921-5207

Figure 4.2b. Total flow entering the H-zone from boreholes D2 - D6 (after Olsson et al., 1991a, p. 49). 


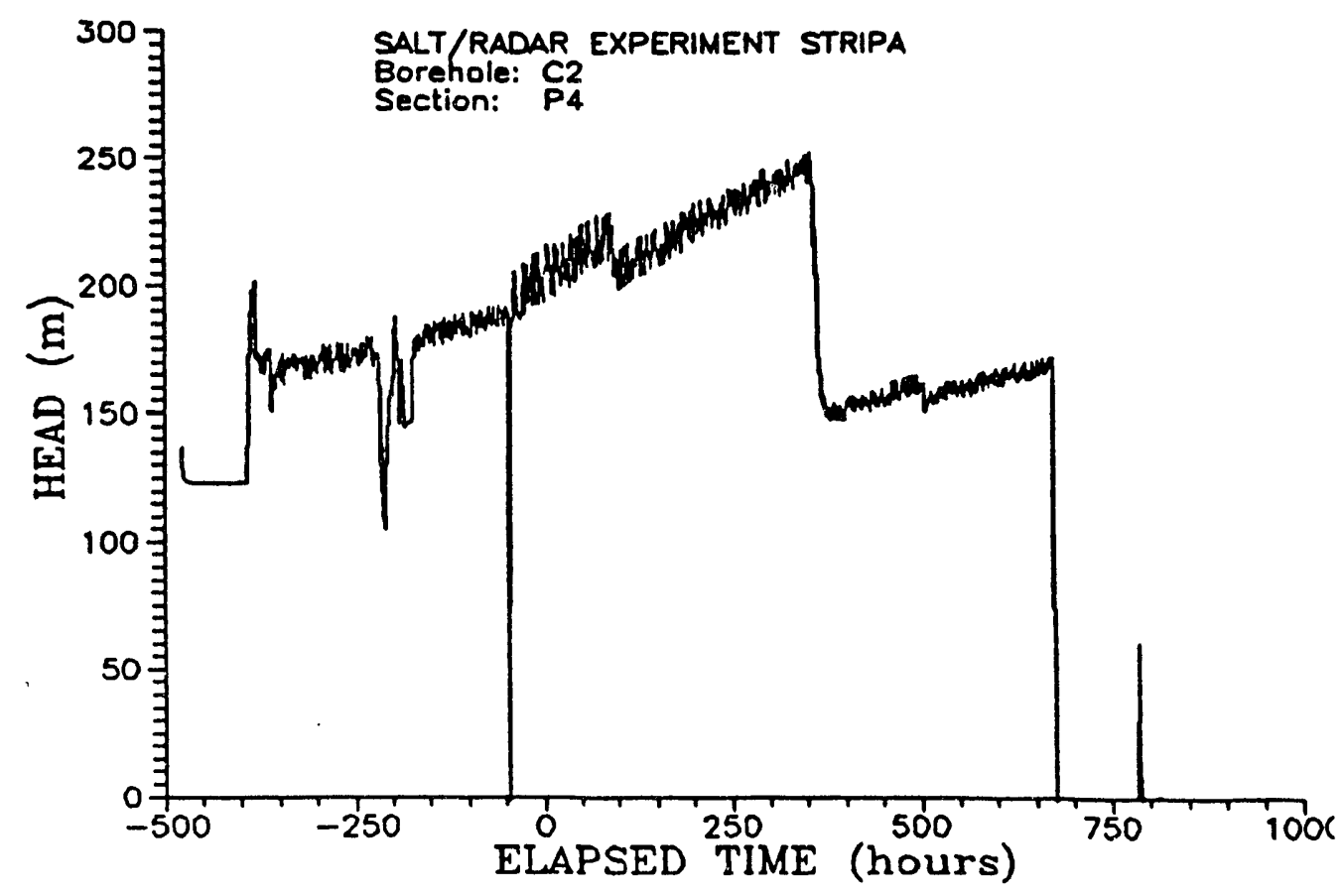

XBL 921-5208

Figure 4.3. Head versus time in the injection interval in C2 (after Olsson et al., 1991a, p. 23). 


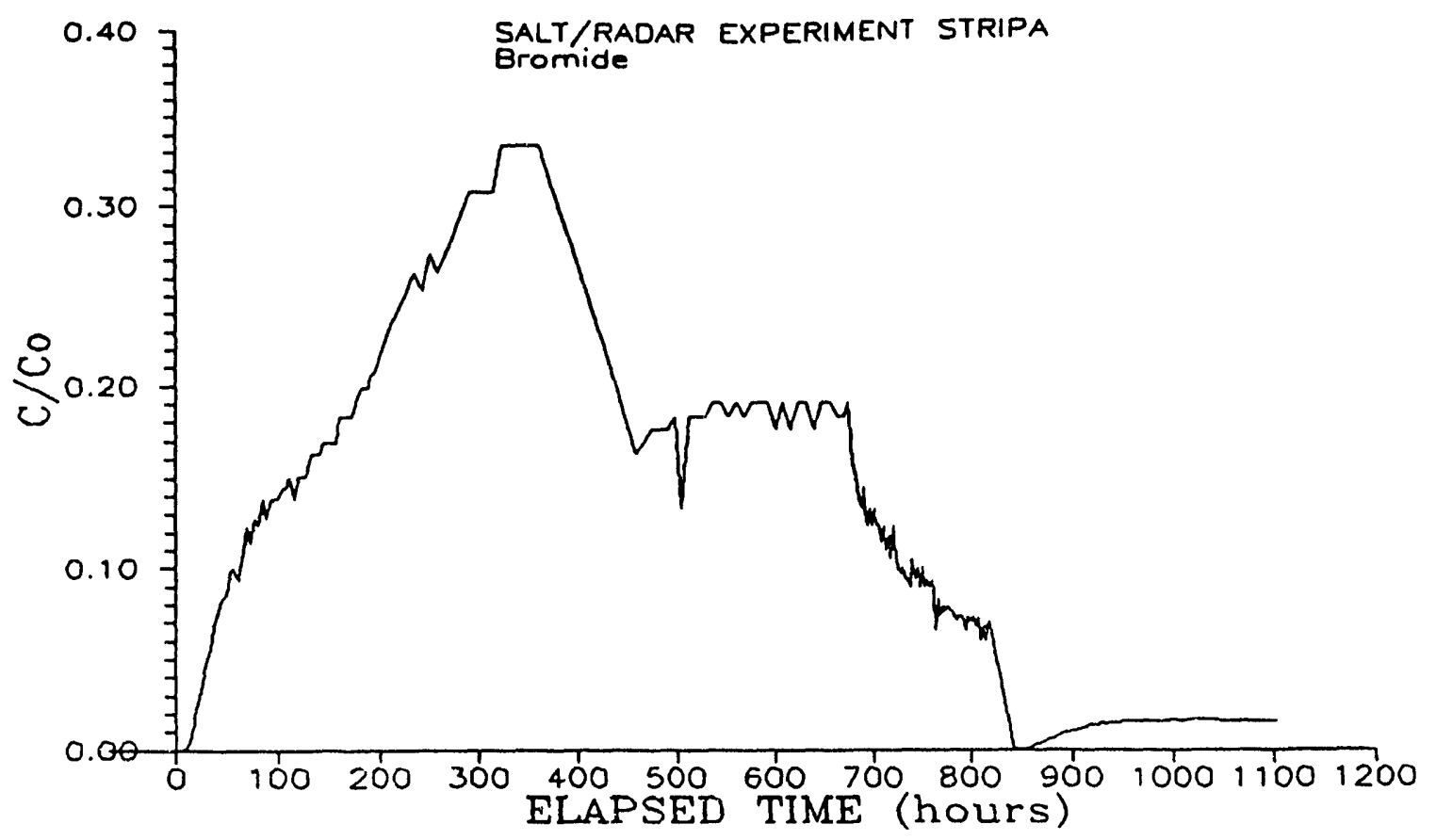

XBL 921-5209

Figure 4.4. Breakthrough of bromide in the total flow from D2-D6 for RSI (after Olsson et al., 1991a, p. 41). 


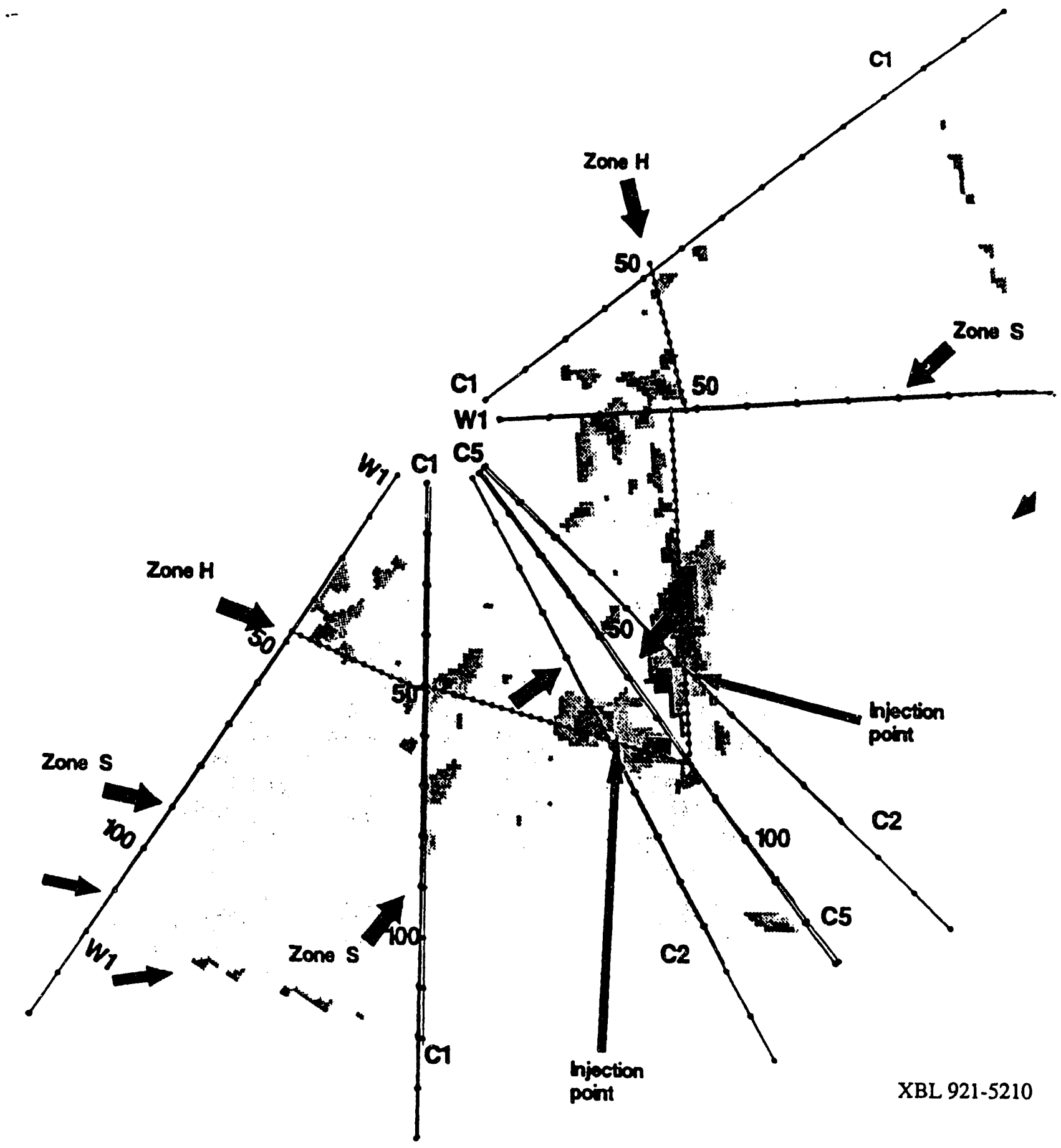

Figure 4.5. Composite of difference tomograms made approximately $290 \mathrm{hrs}$ after start of saline tracer injection for RSI. The injection point is shown by the long arrows. The dotted lines show the trace of the H-zone in the plane of the tomograms (after Olsson, 1991b, p. 142). 


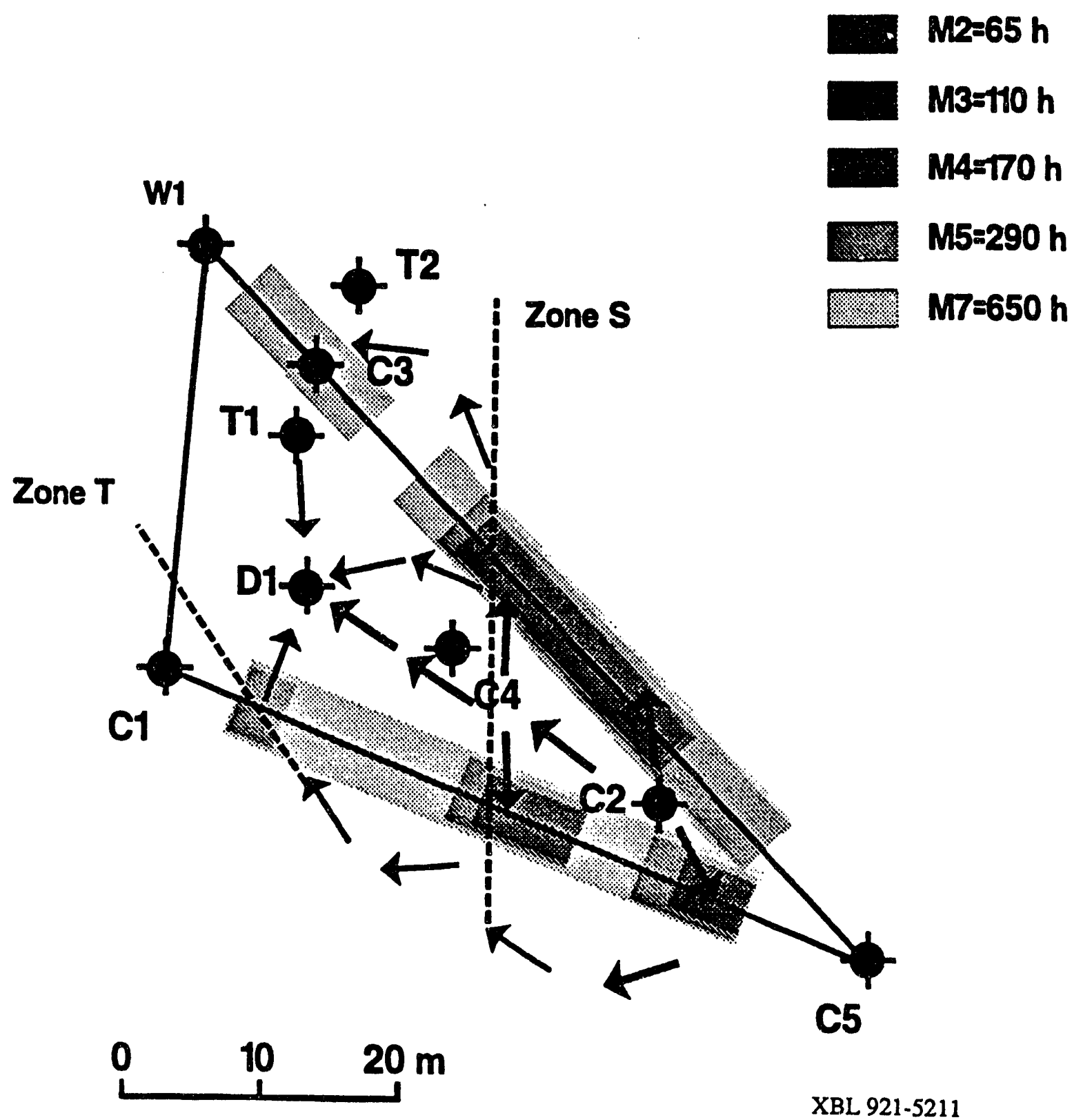

Figure 4.6. Conceptual model of saline tracer flow within the H-zone based on the radar difference tomograms. The boxes indicate when and where saline tracer is observed along the lines of intersection between zone $\mathrm{H}$ anc' the tomogaphic planes (after Olsson, 1991a, p. 99). 
A $2 \%$ saline solution $\left(C_{0}=2 \%\right.$ ) was again injected in $C 2$ at an average rate of $200 \mathrm{ml} / \mathrm{min}$ for just over 200 hours and then reduced. Transport was not simulated beyond this time because the change in boundary conditions led to a transient flow field superimposed on the transport problem. Tracer concentration was monitored in the drift by collection of samples and in $\mathrm{T} 1$ with an electrode. Total flow rate into the drift during this experiment was observed to be $0.13 \mathrm{l} / \mathrm{min}$, a significant increase over the inflow before injection at $\mathbf{C 2}$.

Figure 4.7 shows the injection flow rate versus time for RSII. Time zero refers to the start of tracer injection. Injection rate was very constant for a significant time before the test and for the first $220 \mathrm{~h}$. However, after this point, the injection rate stepped down several times.

Figure 4.8 shows the head versus time in the injection interval. As in Radar/Saline I, the head at injection interval is climbing during the entire experiment. Consequently, the assumption of steady flow conditions incurs some error. Figures 4.9, 4.10 and 4.11 show the breakthrough curves for Bromide in the total inflow to the drift, in $\mathrm{T} 1$ and $\mathrm{T} 2$ respectively. The tracer concentration did plateau in both cases, but after the period of constant injection flow. The highest concentration ratio, $C / C_{0}$, observed was about 0.35 in the drift and about 0.62 in $T 1$. The values are used as an estimate of what the final $C / \mathrm{C}_{0}$ would be if the injection rate had been constant. Figure 4.12 shows a composite attenuation difference tomogram for the three planes at approximately $290 \mathrm{~h}$. Figure 4.13 shows a summary of tracer flow along the intersection between the H-zone and the tomographic planes based on the RSII difference tomography. Two possible minor zones, $S$ and $T$, are shown on the figure.

\subsection{Neretnieks Tracer Tests}

Two types of tracer tests were conducted by Neretnieks et al. (personal communication). The first type consisted of injecting tracer into various borehole intervals and monitoring the arrival in the water collected in the Validation Drift. Injection rates were kept fairly low, but significant. In the second type of test, the hole, $\mathrm{T} 1$ was opened to atmospheric pressure. Tracer was injected into the H-zone from intervals in $\mathrm{T} 2$ and arrivals were monitored in both $\mathrm{T} 1$ and the drift. 


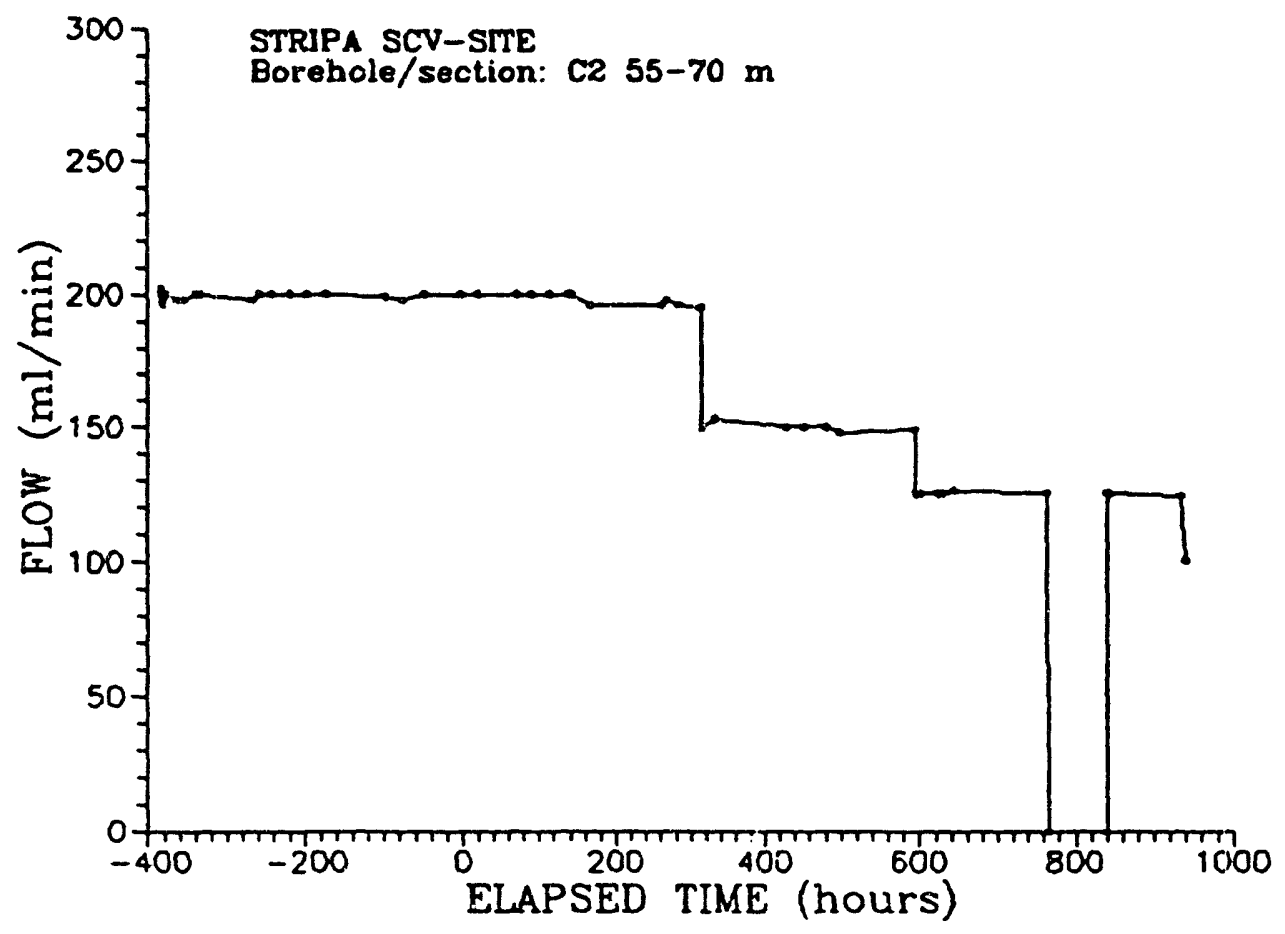

XBL $921-5212$

Figure 4.7. Injection flow rate versus time for Radar/Saline II. The time refers to the start of saline tracer injection (after Olsson, 1991b, p. 35). 


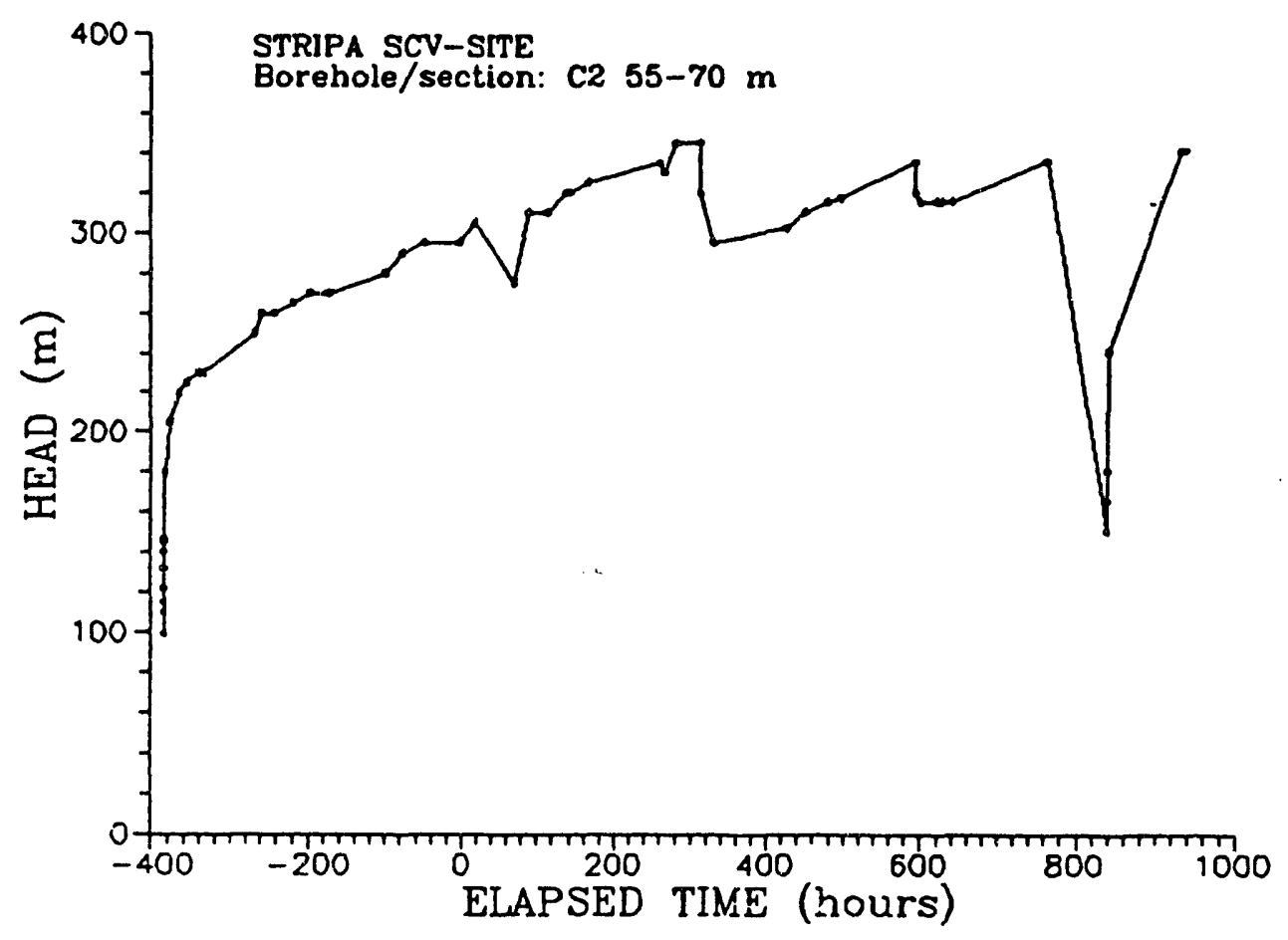

XBL $921-5213$

Figure 4.8. Head versus time in the injection interval in C2 for Radar/Saline II (after Olsson, 1991b, p. 42). 


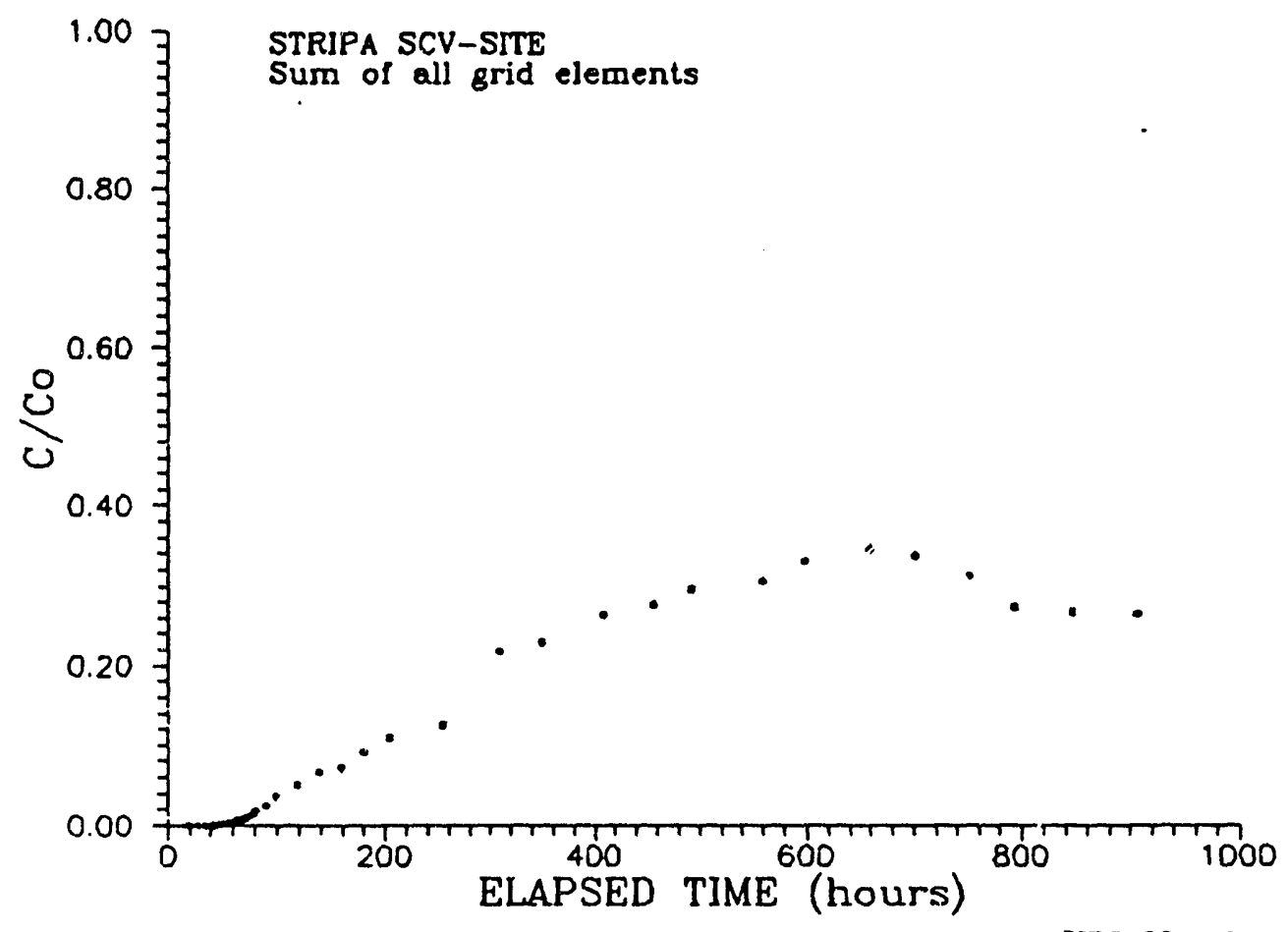

Figure 4.9. Summarized breakthrough in the Validation Drift for RSII (after Olsson et al., 1991b, p. 48). 

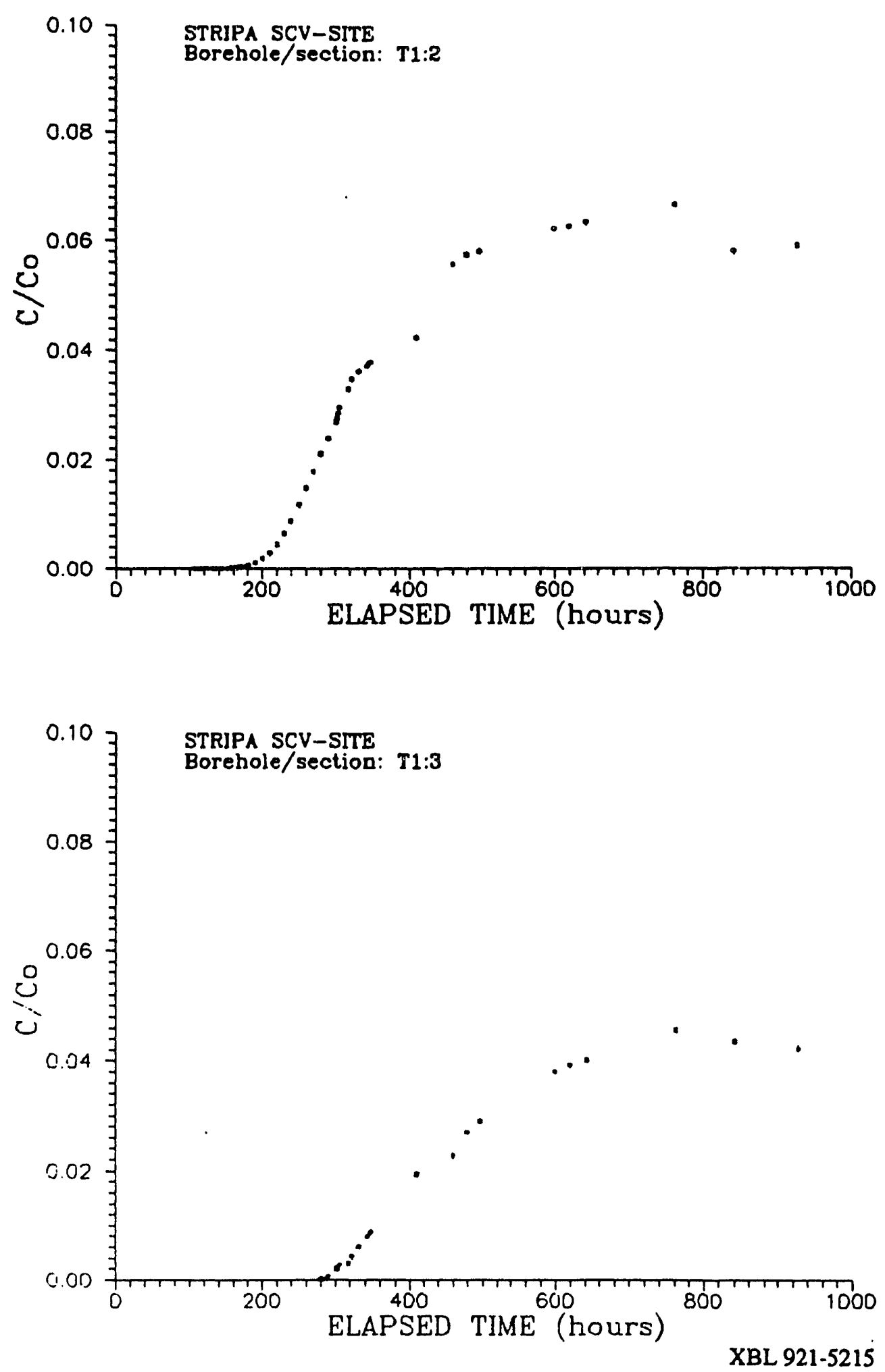

Figure 4.10. Breakthrough of saline tracer in borehole T1, sections 2 and 3 (after Olsson, 1991b, p. D14). 

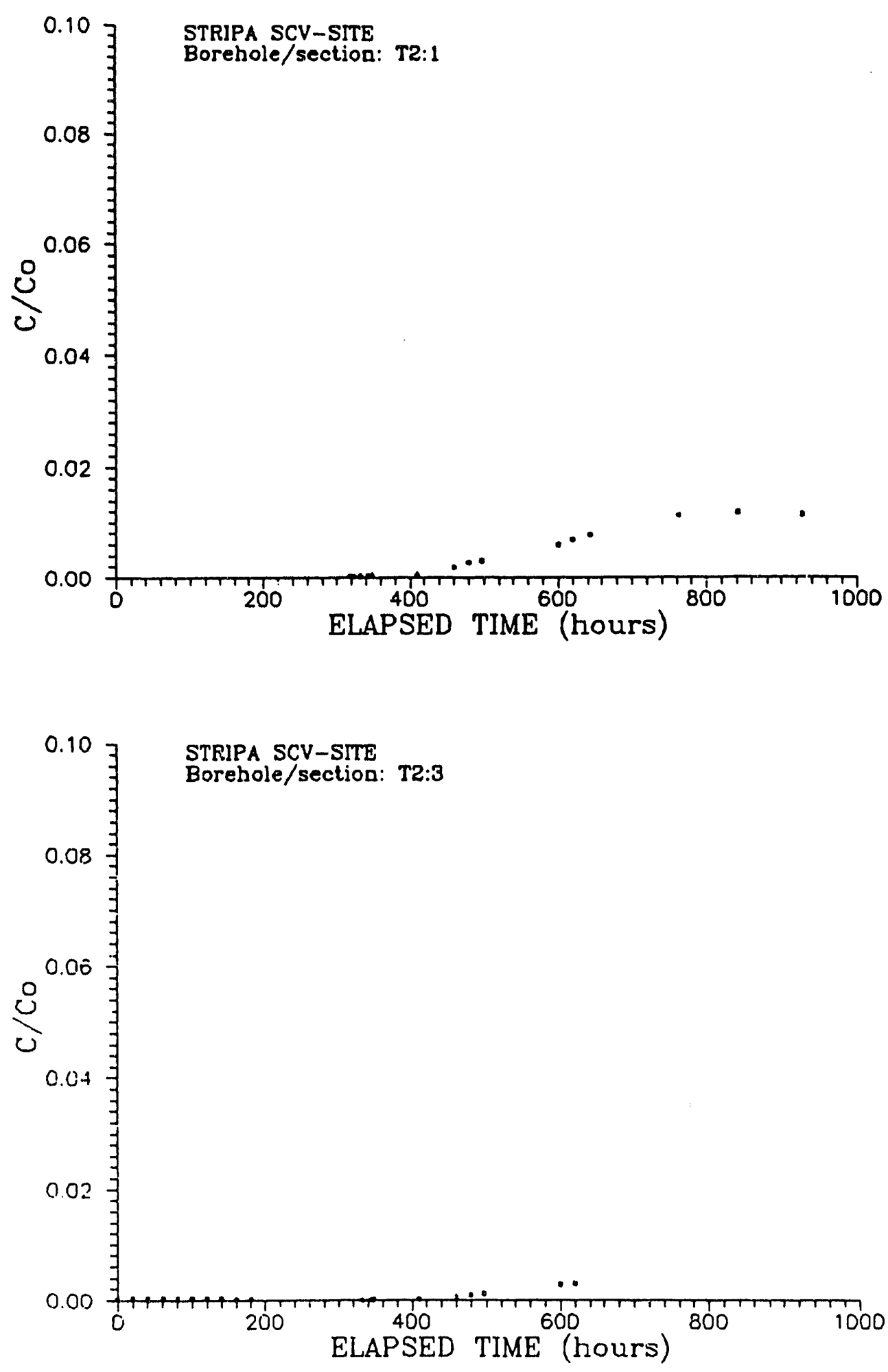

XBL $921-5216$

Figure 4.11. Breakthrough of saline tracer in borehole T2, sections 1 and 3 (after Olsson, 1991b, p. D15). 


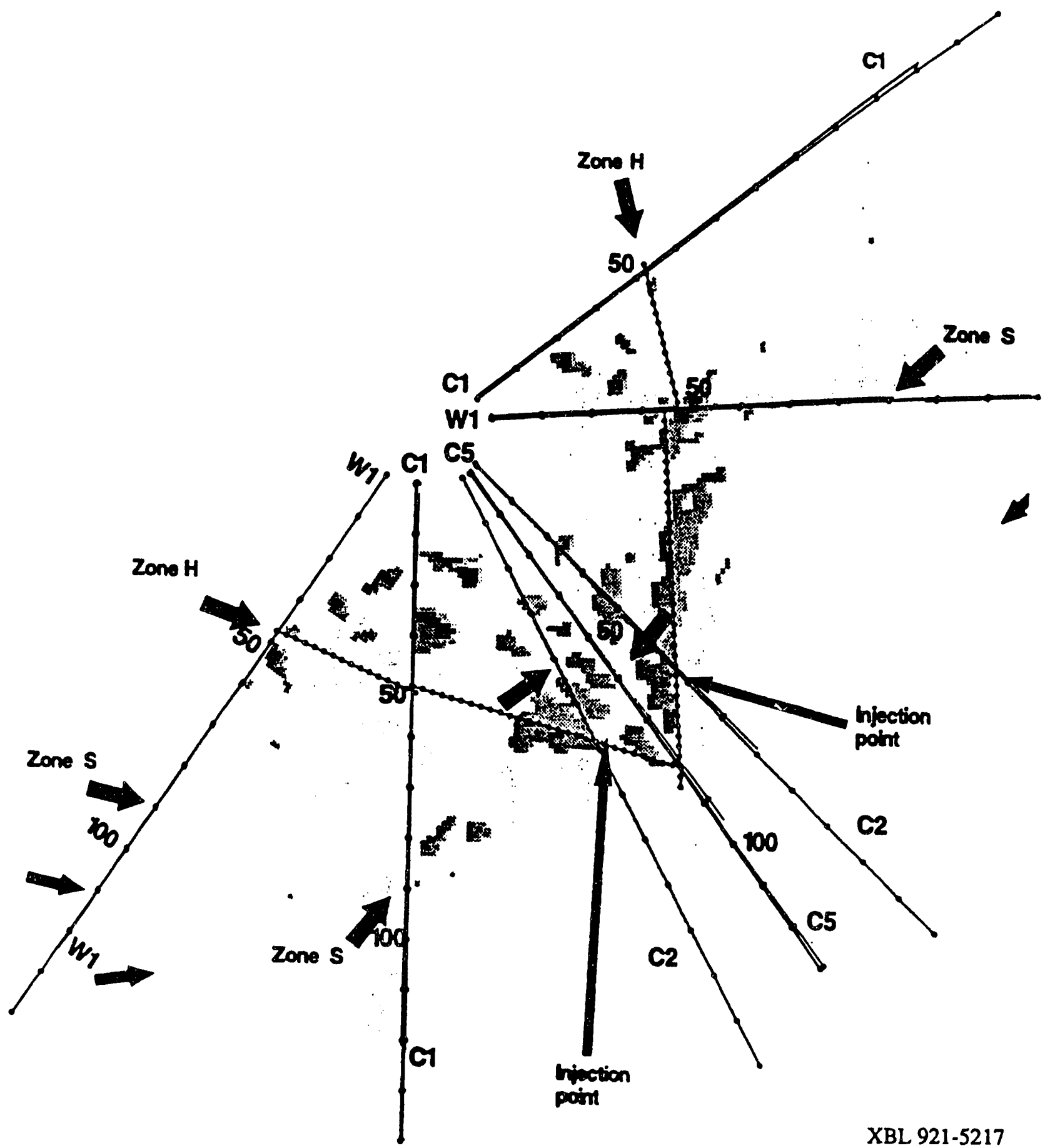

XBL $921-5217$

Figure 4.12. A composite attenuation difference tomogram made approximately $290 \mathrm{~h}$ after tracer injection for Radar/Saline II (after Olsson, 1991b, p. 143). 


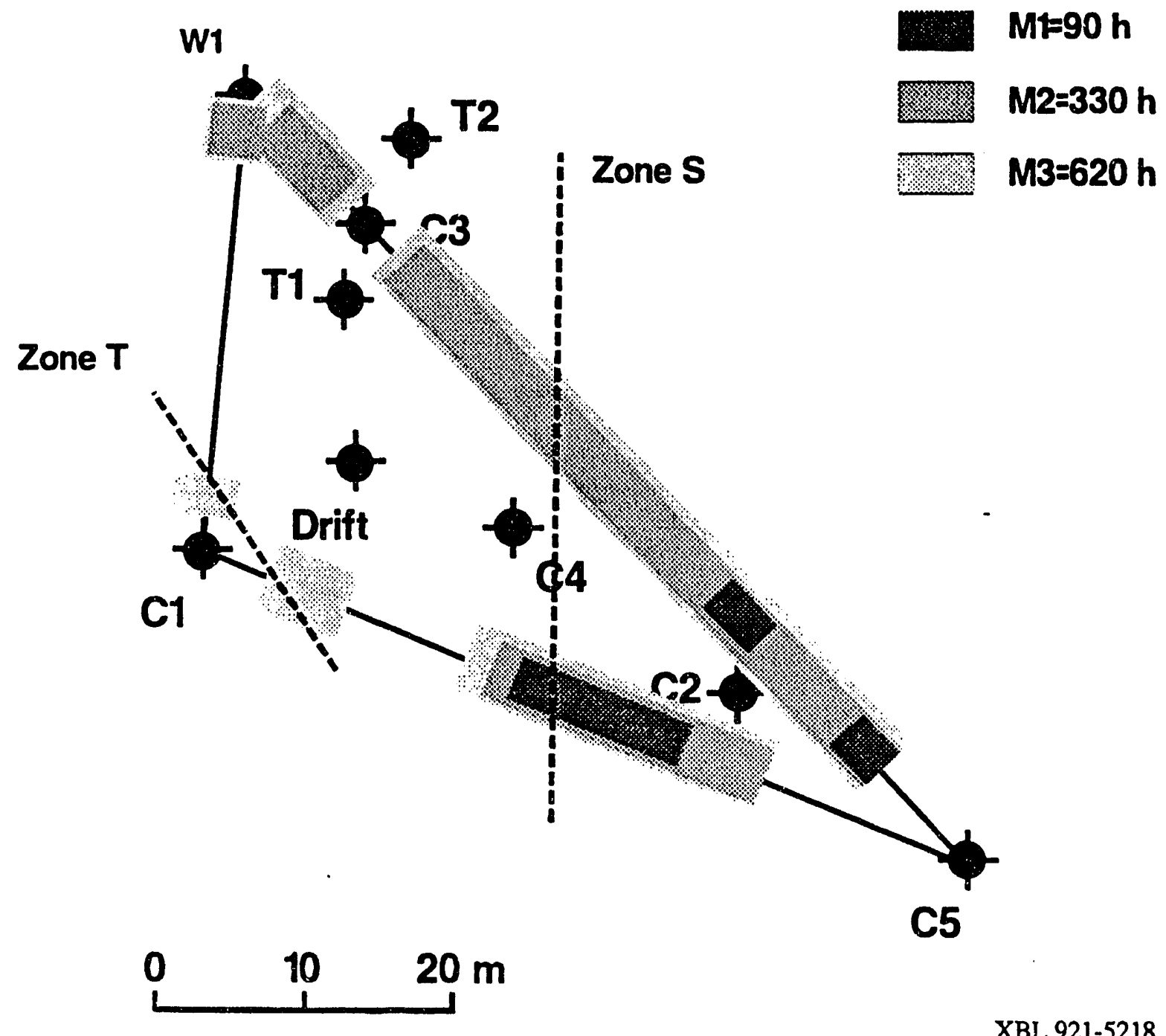

XBL $921-5218$

Figure 4.13. Diagram of attenuation increase in zone $H$. The boxes indicate when and where saline tracer is observed along the lines of intersection between zone $\mathrm{H}$ and the tomogaphic planes (after Olsson, 1991b, p. 131). 
In both cases a complex sequence of injections was used to maximize the number of tests and take advantage of the fact that multiple tracers were available. As mentioned in Section 3.3, the concentration, $\mathrm{C}_{0}$, of the injected tracer was reduced in the simulation when two intervals of the same borehole in the H-zone were injected simultaneously. Injection rates for tracer tests being conducted simultaneously from other boreholes were also included. The table below explains the cases that were modeled as extracted from the specified experimental conditions.

Table 4.1. Conditions modeled to predict the Neretnieks' tracer tests

\begin{tabular}{|c|c|c|c|c|c|c|c|}
\hline \multirow[t]{2}{*}{ Case } & \multirow{2}{*}{$\begin{array}{l}\text { Source } \\
\text { interval }\end{array}$} & \multirow{2}{*}{$\begin{array}{c}\text { Sink } \\
\text { location }\end{array}$} & \multirow{2}{*}{$\begin{array}{c}\text { Injection } \\
\text { rate (total) } \\
\mathrm{ml} / \mathrm{hr}\end{array}$} & \multirow{2}{*}{$\begin{array}{l}\text { Fraction } \\
\text { of Conc. } \\
\text { modeled }\end{array}$} & \multicolumn{2}{|c|}{$\begin{array}{l}\text { Simultaneous } \\
\text { Other Injections }\end{array}$} & \multirow{2}{*}{$\begin{array}{c}\text { Simulation } \\
\text { time } \\
\text { hrs. }\end{array}$} \\
\hline & & & & & Interval & Flow (ml/hr) & \\
\hline $\begin{array}{l}1 \\
2 \\
3\end{array}$ & $\begin{array}{c}\mathrm{T} 1: 2 \\
\mathrm{~T} 2: 1 \\
\mathrm{C} 2\end{array}$ & $\begin{array}{l}\text { drift } \\
\text { drift } \\
\text { drift }\end{array}$ & $\begin{array}{c}4 \\
47.5 \\
15\end{array}$ & $\begin{array}{c}1 \\
30 / 47.5 \\
1\end{array}$ & $\begin{array}{l}\mathrm{T} 2 \\
\mathrm{~T} 1\end{array}$ & $\begin{array}{c}47.5 \\
4\end{array}$ & $\begin{array}{l}2000 \\
2000 \\
2000\end{array}$ \\
\hline 4 & $\mathrm{~T} 2$ & T1, Drift & 56.5 & $30 / 56.5$ & & & 1000 \\
\hline
\end{tabular}

There were three source intervals in each of the T-holes. Thus the notation T1:2 refers to the second interval in the borehole $\mathrm{T} 1$. In case 2 , for example, the source interval was the first interval of the borehole T2. The tracer was actually injected at $30 \mathrm{ml} / \mathrm{hr}$ but another interval of T2 was simultaneously injected at the rate of $17.5 \mathrm{~m} / \mathrm{hr}$. Therefore the value of $\mathrm{C}_{0}$ used in the simulation was reduced by a factor of $30 / 47.5$. Further, $4 \mathrm{ml} / \mathrm{hr}$ were simultaneously injected into T1.

Case 4 used a tracer that had been used previously so that detectable amounts were still in the H-zone when the test started. Also, the hydraulic conditions were not well controlled during this test. 


\subsection{MODELING THE FIRST RADAR-SALINE EXPERIMENT}

The first radar-saline experiment was simulated on both the $\mathrm{C1-2}$ annealed network and the co-annealed network. An injection flow rate of $0.22 \mathrm{~V} / \mathrm{min}$ was applied at the $\mathrm{C} 2$ node. A flow rate of $0.34 \mathrm{~V} / \mathrm{min}$ was applied at the D-holes. Only one breakthrough was obtained to simulate the average breakthrough for all the D-holes.

\subsection{C1-2 Results}

The breakthrough of saline fluid to the D-holes is shown in Figure 5.1 where the value of $A_{E}$ has been adjusted such that the breakthrough curve gives the best fit (by eye) to the data and dispersivity is zero. The figure shows that the simulated $C / C_{0}$ curve levels out below the values observed. The reason for this can be seen in Figure 5.2 which shows a snapshot of tracer concentration in the configuration at approximately $277 \mathrm{hrs}$. The simulated values of $\mathrm{C} / \mathrm{C}_{0}$ were too low because there were too many pathways; too much advective dispersion and too much flow to $\mathrm{Z}$ shaft.

\subsection{Co-annealed Results}

The breakthrough of saline fluid to the D-holes is shown in Figure 5.3 where the value of $A_{E}$ has been adjusted such that the breakthrough curve gives a good estimate of the first arrival and dispersivity is zero. The figure shows that the simulated values of $\mathrm{C} / \mathrm{C}_{0}$ level out far above the observed values of $\mathrm{C} / \mathrm{C}_{0}$. The reason for this can be seen in Figure 5.4 which shows a snapshot of tracer concentration in the configuration at approximately $277 \mathrm{hrs}$. The simulated values of $\mathrm{C} / \mathrm{C}_{0}$ were too high because there are too few pathways; too little advective dispersion and no flow to Z-shaft.

To some extent it is encouraging that these two simulations bracket the actual behavior because it may indicate that a sufficient number of configurations could provide a good estimate 


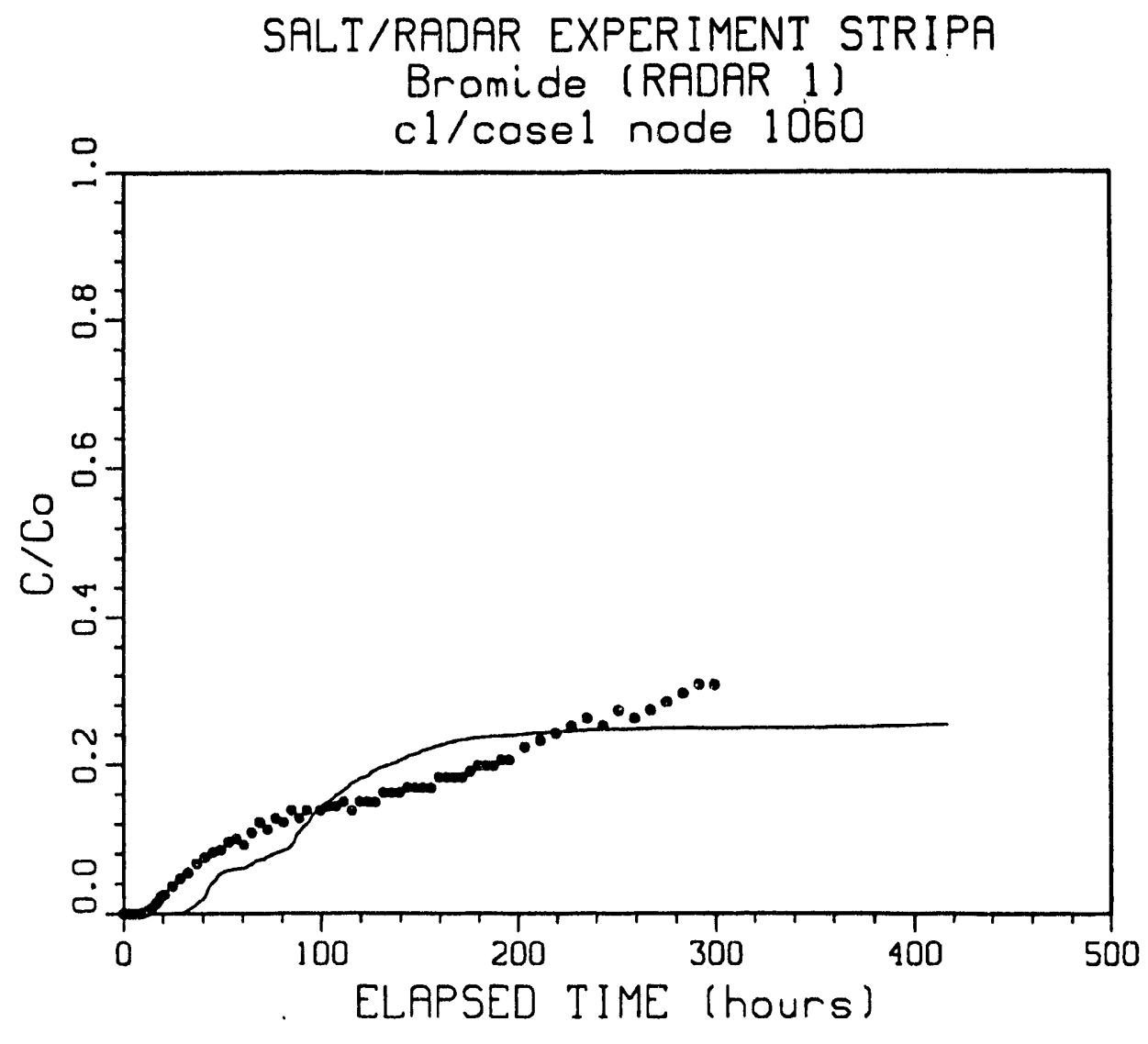

Figure 5.1. Simulated tracer breakthrough at the D-holes for RSI plotted against the data for the simulation period of $400 \mathrm{hrs}$ calculated using the C1-annealed configuration. Dots are data; the line is the simulation. 


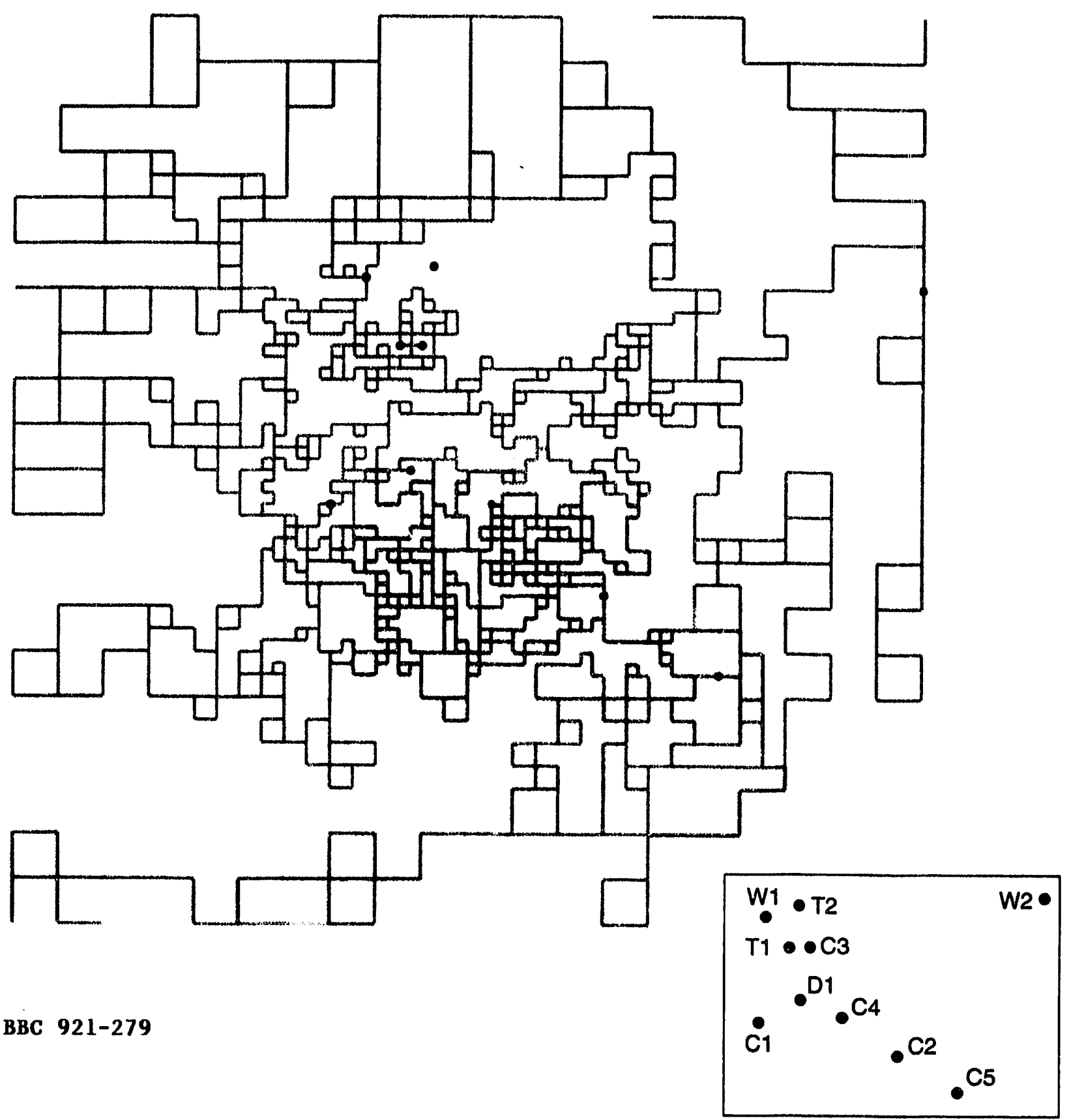

Figure 5.2. A snapshot of concentration in the C1-annealed configuration at approximately $270 \mathrm{hrs}$. for RSI. The wells are shown by black dots. Dark blue is high concentration and red is zero. This figure can be compared to Figure 4.6. 


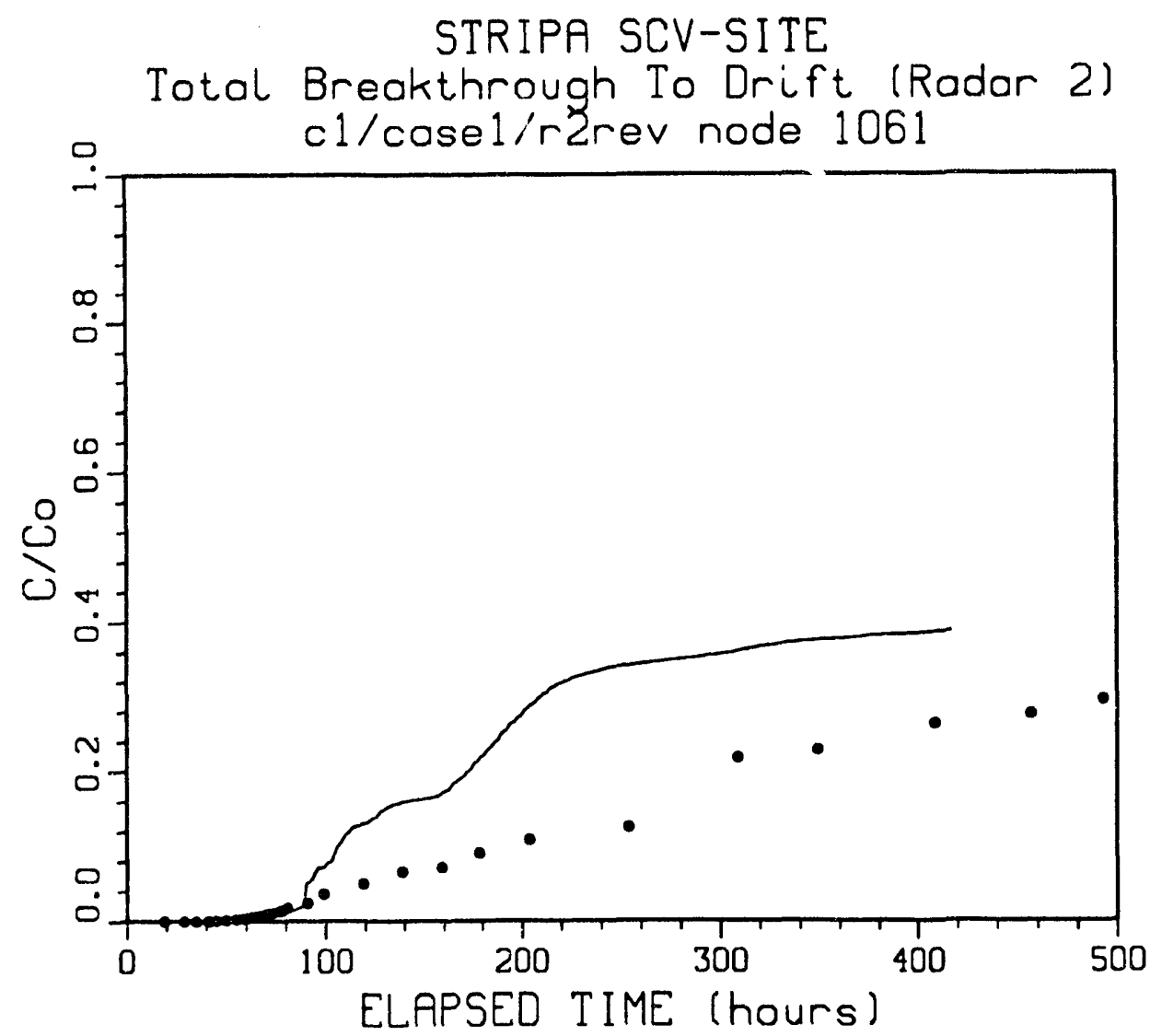

XBL $921-5221$

Figure 5.3. Simulated tracer breakthrough at the D-holes for RSI plotted against the data for the simulation period of $300 \mathrm{hrs}$ calculated using the cu-annealed configuration. Dots are data; the line is the simulation. 


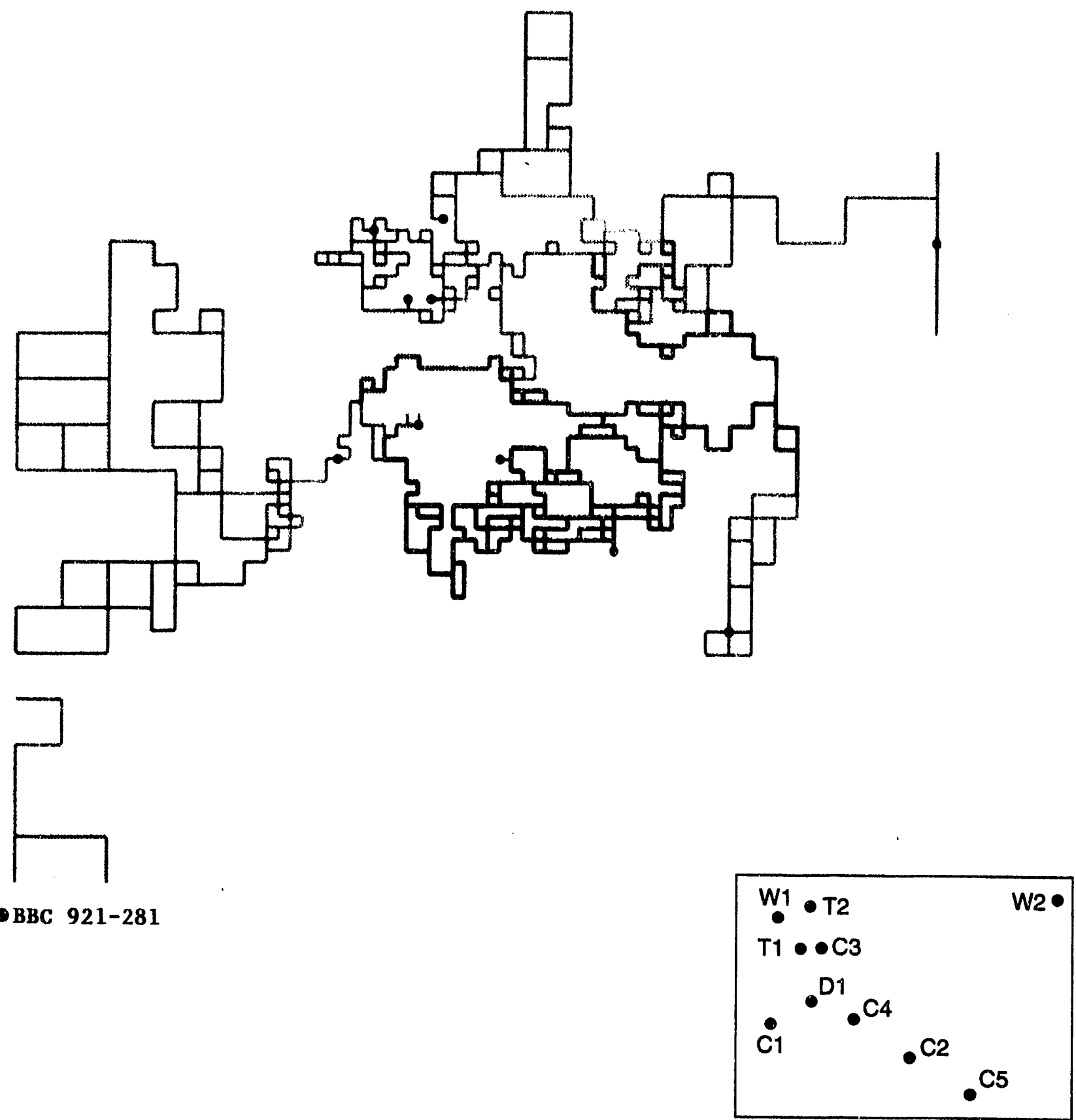

Figure 5.4. A snapshot of concentration in the co-annealed configuration at approximately $270 \mathrm{hrs}$. for RSI. The wells are shown by black dots. Dark blue is high concentration and red is zero. This figure can be compared to Figure 4.6. 
of tracer behavior. If the interference tests were used to develop a serizs of configurations, it seems likely that a configuration could be found which matched the RSI breakthrough. As an altemative a better match was sought by increasing the dispersivity in the model available. The value of $\mathrm{C} / \mathrm{C}_{0}$ is already too low in the $\mathrm{C} 1-2$ configuration. Consequently, only the co-annealed configuration was modified as explained below.

\subsubsection{Co-annealed Configuration with a Background Permeability}

The annealing process used in Long et al. (1992) consists of networks where the elements are either tumed "off" or "on". If all the "off" elements are actually conductive, but much less conductive than the "on" elements, the hydraulic behavior will be essentially unaffected. However, these low conductance elements may disperse tracer. To that end, small conductances, $K_{M}$, were assigned to the "off" elements. Values of $K_{M} / \mathrm{K}$ from $10^{-6}$ to $10^{-2}$ were tried, however the change in the breakthrough curve was insignificant. Essentially, the low conductance elements do not change the "corridor" and thus the breakthrough is unaffected.

\subsubsection{Co-annealed with Non-zero Dispersivity}

Next, the elements in the co-annealed configuration were given a non-zero dispersivity. Without any dispersivity in the network elements, the dead-end elements in the network play no role in flow or transport. Thus, in the base cases described above, the dead ends are eliminated. Consequently, the first try at non-zero dispersivity did not include the dead-end elements. Essentially this just increased the rate of tracer arrival at the D-holes because tracer could only disperse along the existing corridors. The breakthrough curve was still too steep. A better match was achieved by including the dead-ends. In this case, the tracer can disperse into the dead-ends and thus lower the concentration observed at the D-holes. It is possible to match breakthrough time and slope very well, but tracer is dispersed everywhere.

\subsection{Conclusions}

As a result of these calculations, the remaining simulations were conducted using a zero dispersivity and using both the C1-2 and co-annealed case. This was done for three reasons. First, 
the two simulations did bracket the observed behavior of RSI, the two configurations might bracket the remaining transport behavior. Second, using dispersivity to obtain a match resulted in behavior that was counter-indicated by the radar results. The tomograms indicated that tracer was fairly localized within the H-zone. The simulations with significant dispersivity spread the tracer out. Thus matching the breakthrough with dispersivity seems very unrealistic. It is more likely that the actual network provides advective dispersion somewhere in between the two configurations we have available. Third, although tracer data can almost always be matched by turning "knobs," this knob tuming distracts from seeing exactly how much can be leamed about tracer transport from the network configuration alone. All remaining tracer simulations are done: using the $\mathrm{Cl}$ and co-annealed configurations with zero dispersivity in the network elements. Further, the values of $A_{E}$ are used in the above breakthrough curves. This is the only calibration employed in the predictions. 


\subsection{MODELING THE SECOND RADAR-SALINE EXPERIMENT}

The second radar-saline experiment was simulated on both the C1-2 annealed network and the co-annealed network. An injection flow rate of $0.21 /$ min was applied at the C2 node. A flow rate of $0.13 / \mathrm{min}$ was applied at the Validation Drift. Thus the drift is a much weaker sink than the D-holes were in RSI. Only one breakthrough was obtained to simulate the average breakthrough for all parts of the drift.

\subsection{C1-2 Results}

The breakthrough of saline fluid to the Validation Drift is shown in Figure 6.1 where the value of $A_{E}$ is the same as in Figure 5.1. This simulation does a good job of matching the first arrival, but the figure shows that the simulated values of the $C / C_{0}$ curve now level out well above the observed values. In RSI, the reverse was true: the simulated values of $\mathrm{C} / \mathrm{C}_{0}$ never got as high as the final observed value. This change in the relative positions of the data and the simulated values between RSI and RSII is surprising. Contrary to the model results, the data indicates that there is not much change in the plateau value of $C / C_{0}$, but a significant increase in first arrival time caused by a lower strength sink.

To examine why $\mathrm{C} / \mathrm{C}_{0}$ is higher for the simulation of RSII than for RSI; note that the drift is a weaker sink than the D-holes and consequently the flow rate to the drift is less that that to the D-holes. A constant flow boundary condition was used at the D-holes and the Drift in the simulation of RSI and RSII. The injection rate of tracer is the same in both simulations. Consequently, in the simulation the injected water is a greater proportion of the inflow in RSII than in RSI. Thus a greater proportion of tracer gets to the Drift than to the D-holes.

Figure 6.2 shows a snapshot of the simulated tracer concentration for RSI in the C1 configuration at approximately $347 \mathrm{hrs}$. There is little difference between Figures 5.2 and 6.2 . The concentration distribution shown in Figure 6.2 does not completely agree with the RSII 


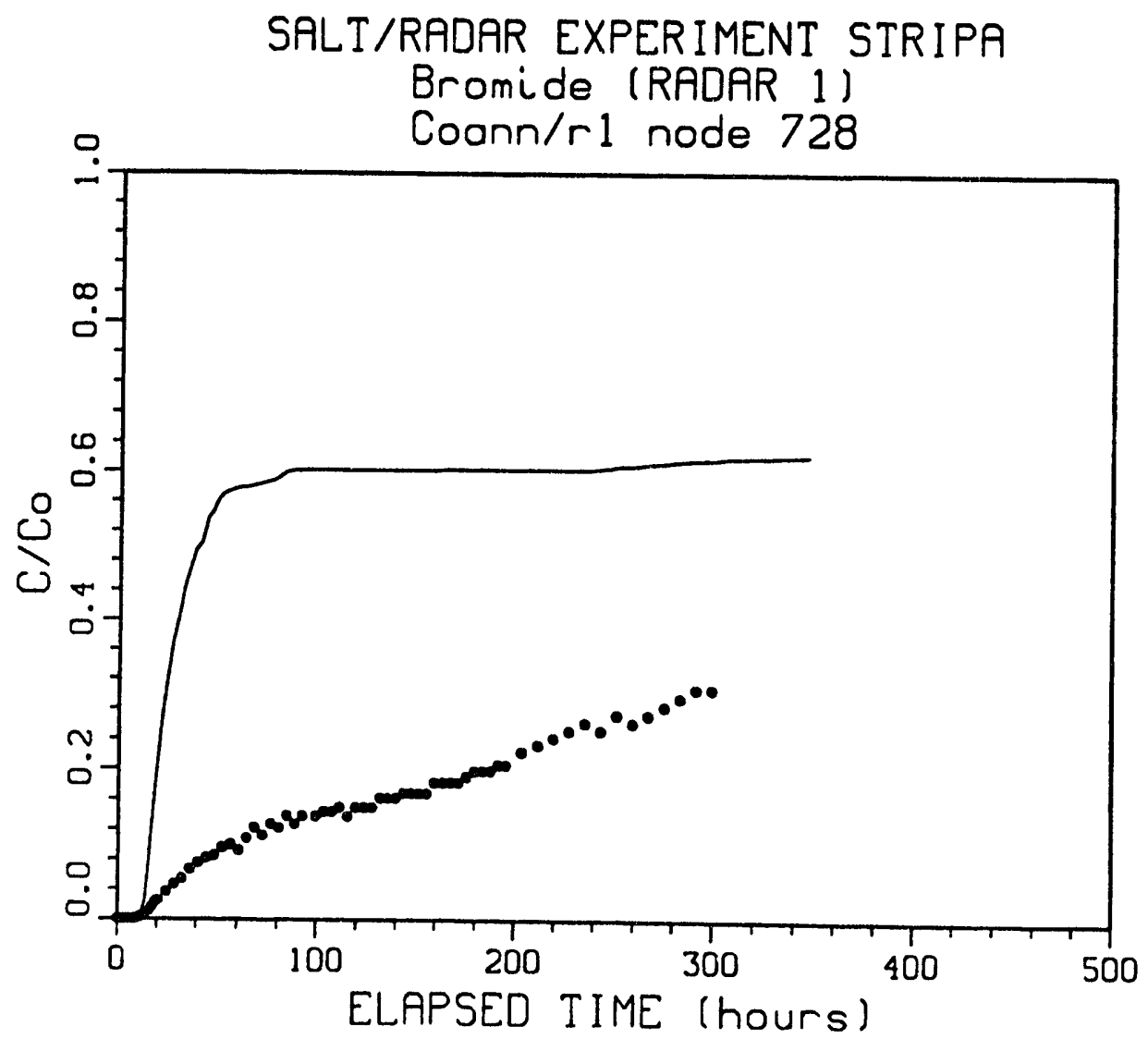

XBL 921-5220

Figure 6.1. Simulated tracer breakthrough at the Validation Drift for RSII plotted against the data for the simulation period of $400 \mathrm{hrs}$ calculated using the $\mathrm{Cl}$-annealed configuration. Dots are data; the line is the simulation. 
tomographic data shown in Figure 4.13. The simulated tracer in Figure 6.2 is much less concentrated in the vicinity of the injection well and does not reach the vicinity of the T-holes. Therefore no breakthrough curves were obtained from the T-holes to compare with the data.

\subsection{Co-annealed Results}

The breakthrough of saline fluid to the Validation Drift for the co-annealed configuration is shown in Figure 6.3 where the value of $A_{E}$ is the same as for RSI shown in Figure 5.3. The curve shows that the first arrival matches very well. The simulated values of $C / C_{0}$ for RSII again level out far above the values for the RSI simulation and higher than those observed for RSII. Essentially, the same trend in this simulation as in the RSII simulation is seen using the $\mathrm{Cl}$ configuration. These simulations show the proportion of tracer to water increasing as the total flow to the drift decreases, but the data shows this proportion is relatively unaffected by the change in conditions induced by the drift.

Figure 6.4 shows the snapshot of concentration for the co-annealed configuration for RSII at about $347 \mathrm{hrs}$. Figure 6.4 for the co-annealed case compares qualitatively to Figure 4.13 better than Figure 6.2 for the $\mathrm{C} 1$ case. Tracer is less smeared out near the injection hole and does reach the vicinity of the T-holes. However, local vagaries of the mesh near the T-holes prevent tracer from arriving at these locations, so again, there is no simulated breakthrough data for the T-holes.

\subsection{Conclusions}

The data from RSI was used to choose a value of $A_{E}$ to calibrate the transport models. Then the calibrated models were used to predict the results of RSII. These calibrated models reasonably predicted first arrival but over predicted the maximum $C / C_{o}$ for $R S I I$. For the $C 1$ case, if dispersivity was added to match $C / C_{0}$ for $R S I$ the model would still have overpredicted $C / C_{0}$ for RSII. If dispersivity was added to the co-annealed case, the model might have been able to match RSII but the addition of dispersivity would have made the RSI simulation worse. Adding dispersion would not allow us to match both RSI and RSII. 


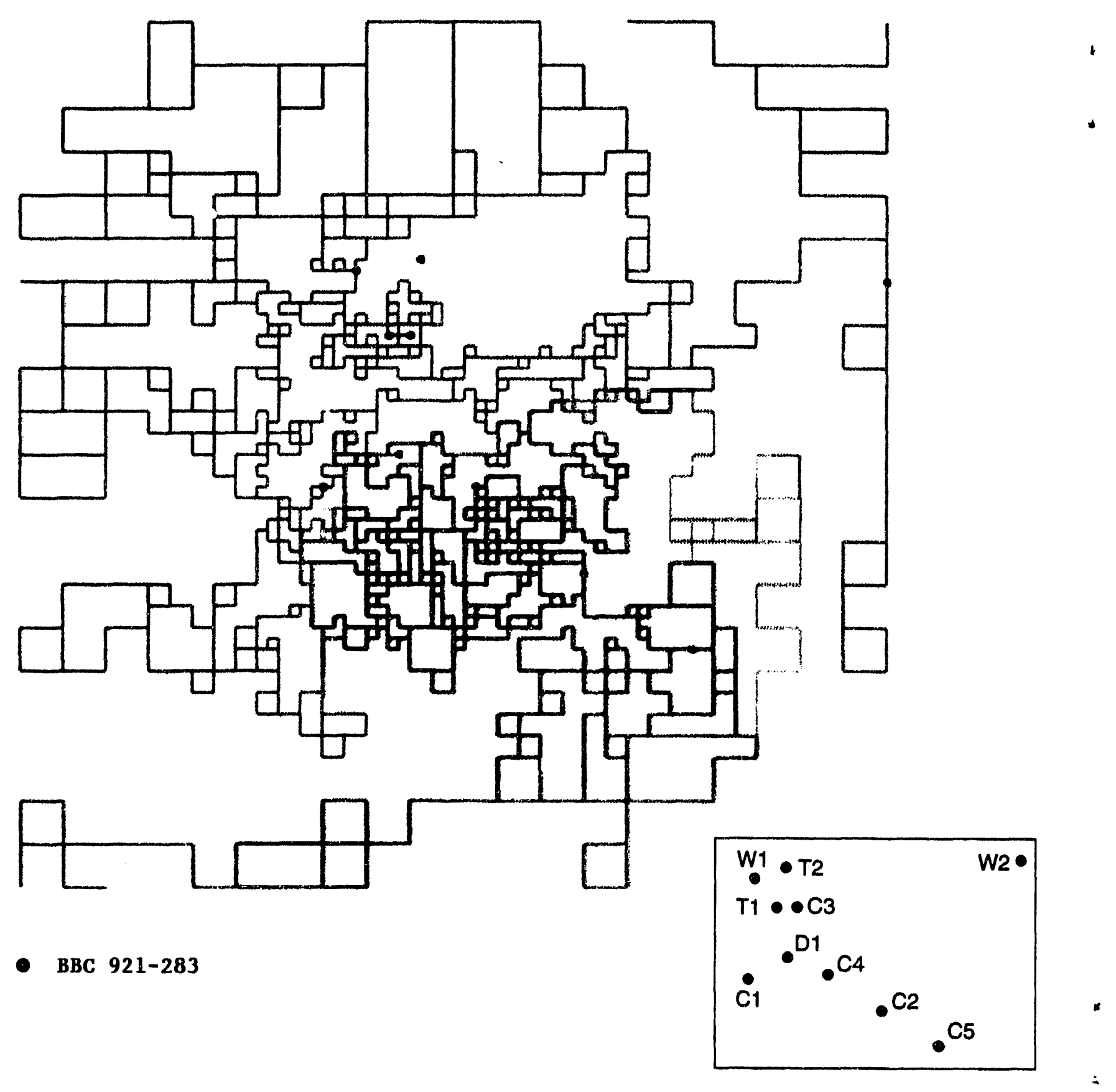

Figure 6.2. A snapshot of concentration in the C1-annealed configuration at approximately $347 \mathrm{hrs}$. for RSII. The wells are shown by black dots. Dark blue is high concentration and red is zero. This figure can be compared to Figure 4.13. 


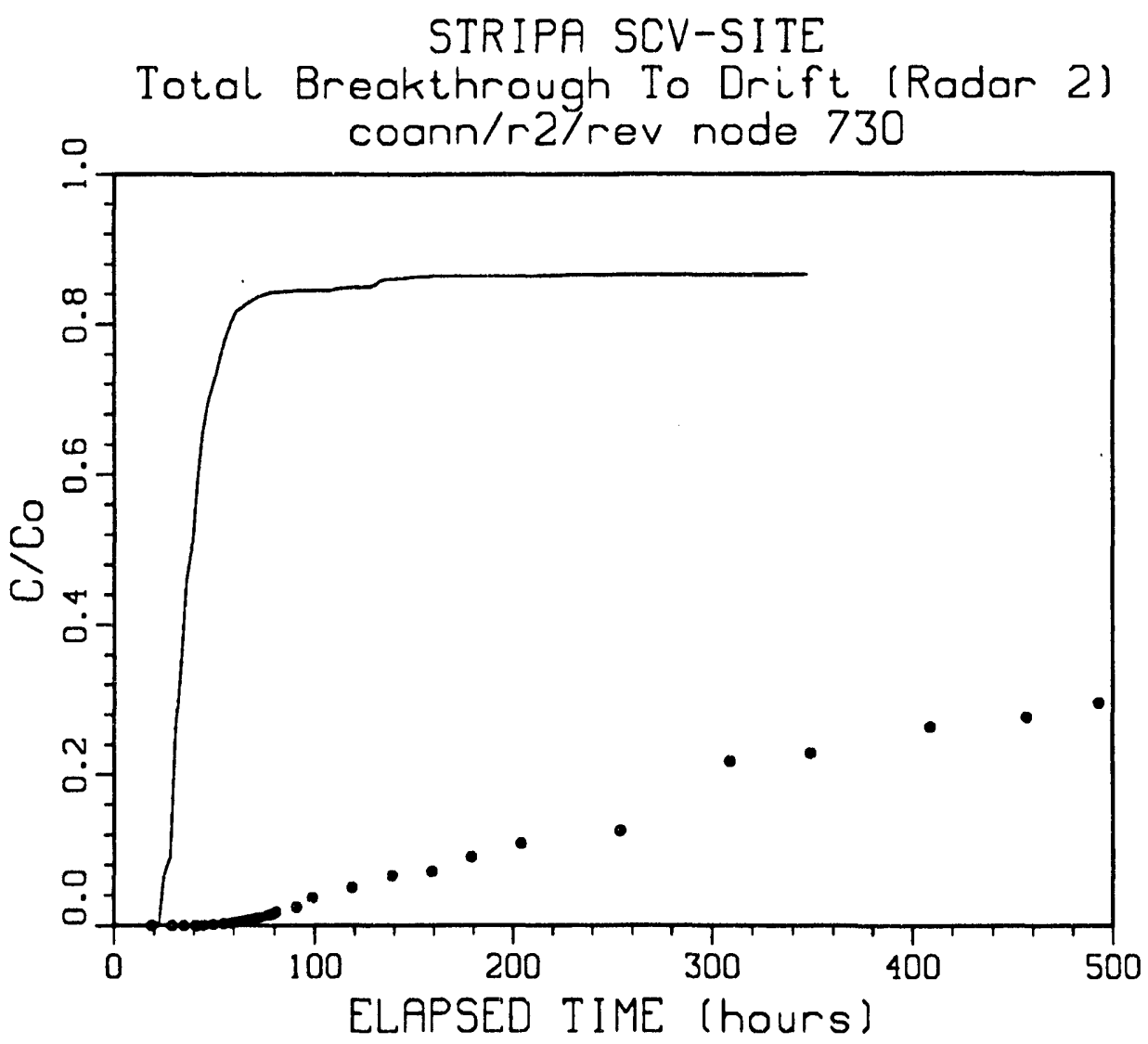

XBL $921-5222$

Figure 6.3. Simulated tracer breakthrough at the Validation Drift for RSII plotted against the data for the simulation period of $300 \mathrm{hrs}$ calculated using the co-annealed configuration. Dots are data; the line is the simulation. 

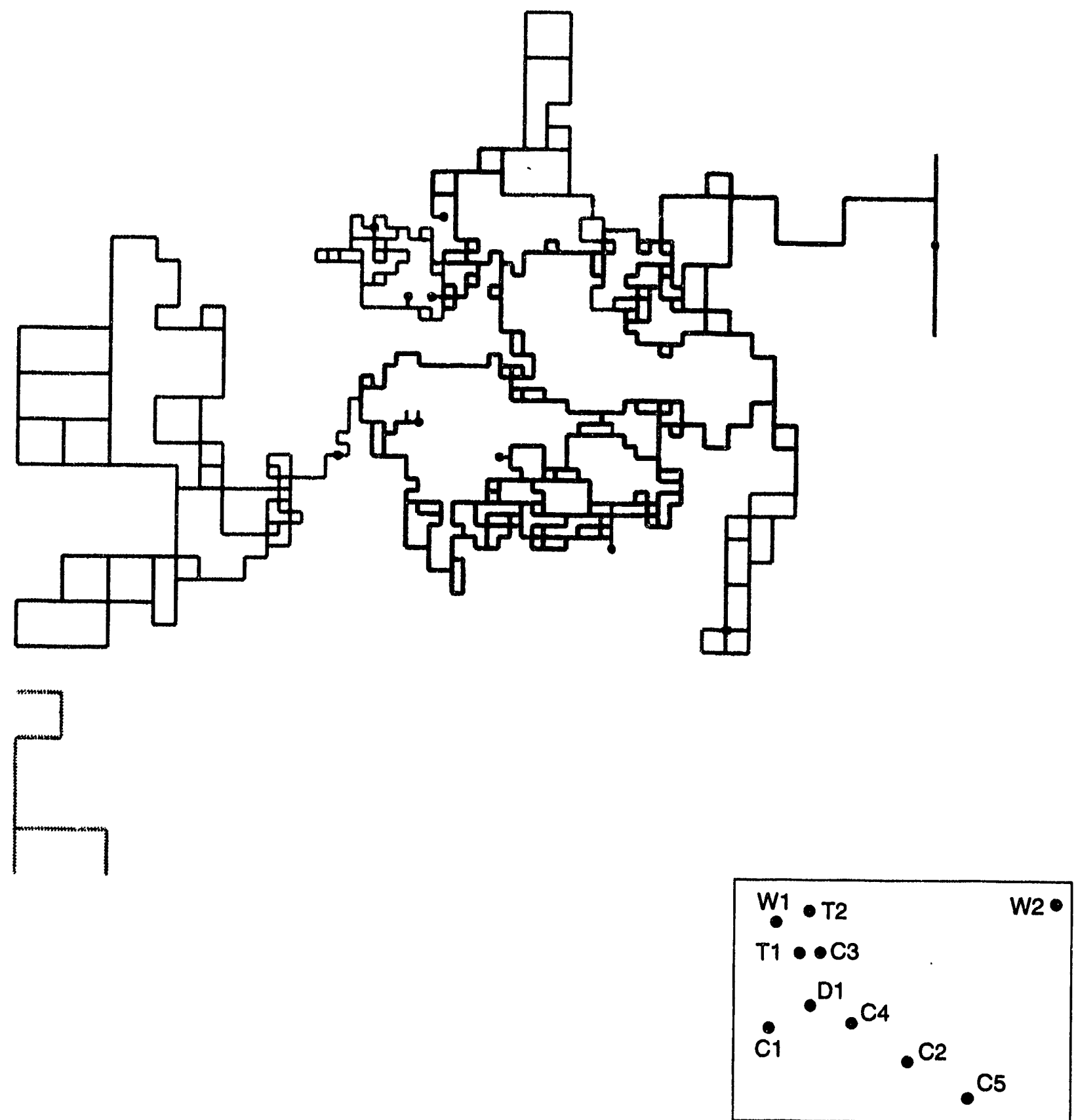

Figure 6.4. A snapshot of concentration in the co-annealed configuration at approximately $347 \mathrm{hrs}$. for RSII. The wells are shown by black dots. Dark blue is high concentration and red is zero. This figure can be compared to Figure 4.13. 


$$
-55-
$$

The comparison of the data and these simulations underscores the fact that the hydrologic conditions in the vicinity of the drift were not well understood and there was a poor understanding of the drawdowns imposed by the drift. These conditions have a significant effect on the tracer transport. 


\subsection{PREDICTIONS OF TRACER TRANSPORT}

Four tracer test predictions (called cases 1 through 4) were made as described in Table 4.1. These are divided into two groups: The first group consists of tracer transport from various sources to the drift. The second group is one case of transport from T2 when T1 is open to atmospheric pressure. Each of these predictions were simulated twice: once with the $\mathrm{Cl}-2$ configuration and once with the co-annealed configuration as described in Chapters 5 and 6. No dispersion coefficient was used. The calculated breakthrough curves and selected concentration snapshots are given for these cases below.

\subsection{Predictions of Transport to the Drint}

The following simulations were made for transport from $T 1, T 2$ and $C 2$ to the drift.

\subsubsection{Case 1: Transport from $\mathrm{T} 1$}

Figure 7.1 shows the predicted breakthrough to the drift for injection into $\mathrm{T} 1$ for the $\mathrm{Cl-2}$ configuration. The predicted breakthrough time is about $800 \mathrm{hrs}$ and the actual breakthrough time is closer to $400 \mathrm{hrs}$. The predicted maximum $\mathrm{C} / \mathrm{C}_{0}$ is about one third of the actual value. This is in agreement with the RSI simulation and the fact that Figure 7.2 shows that most of the tracer escapes around the drift to the Z-shaft.

Figure 7.3 shows the predicted breakthrough to the drift for injection into $\mathrm{Tl}$ for the coannealed configuration. The predicted breakthrough time is even greater in this case and the maximum value of $\mathrm{C} / \mathrm{C}_{0}$ too high, as might be expected from the $\mathrm{RSI}$ simulation. A high value of $\mathrm{C} / \mathrm{C}_{0}$ is in agreement with Figure 7.4 which shows that none of the tracer escapes to the Z-shaft.

\subsubsection{Case 2: Transport from $T 2$}

Figure 7.5 Shows the predicted breakthrough to the drift for injection into T2. The actual 


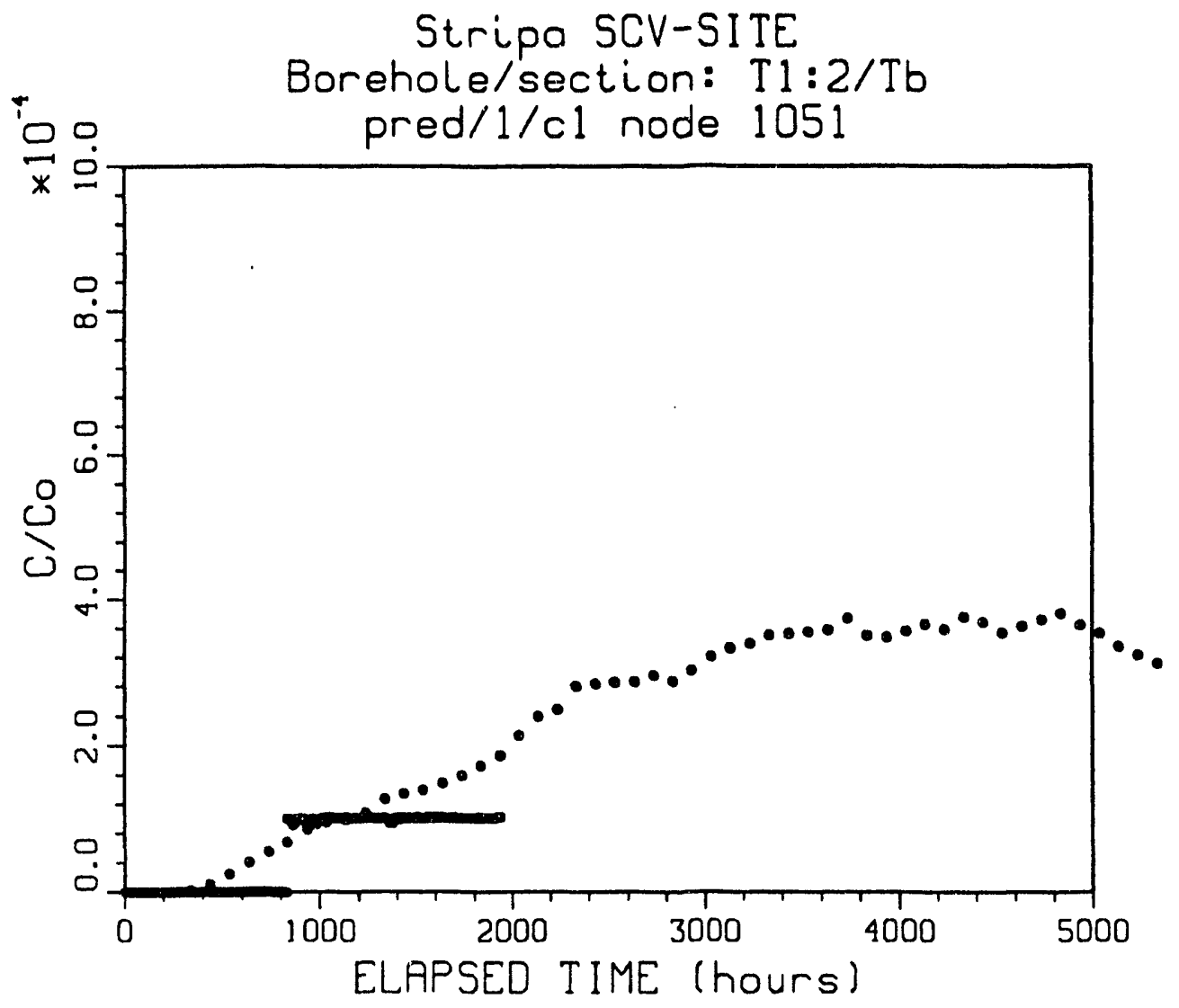

XBL $921-5223$

Figure 7.1. Predicted breakthrough into the Validation Drift from $\mathrm{T} 1$ for the C1-2 configuration. The data is shown by dots, and the simulation by the solid line. 


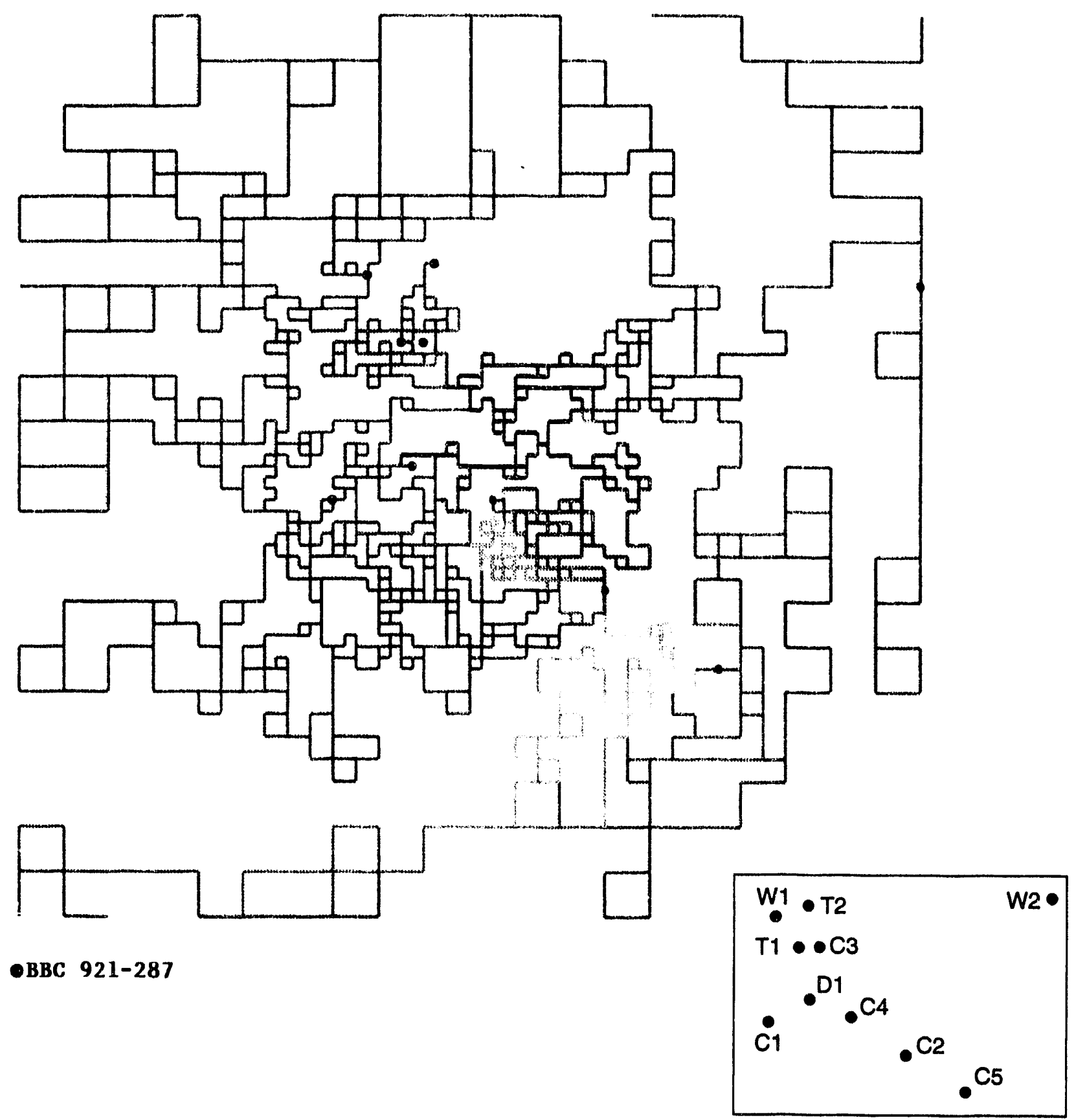

Figure 7.2. Snapshot of concentration for Case 1, injection in $\mathrm{T} 1$ for the $\mathrm{C} 1$ configuration. 


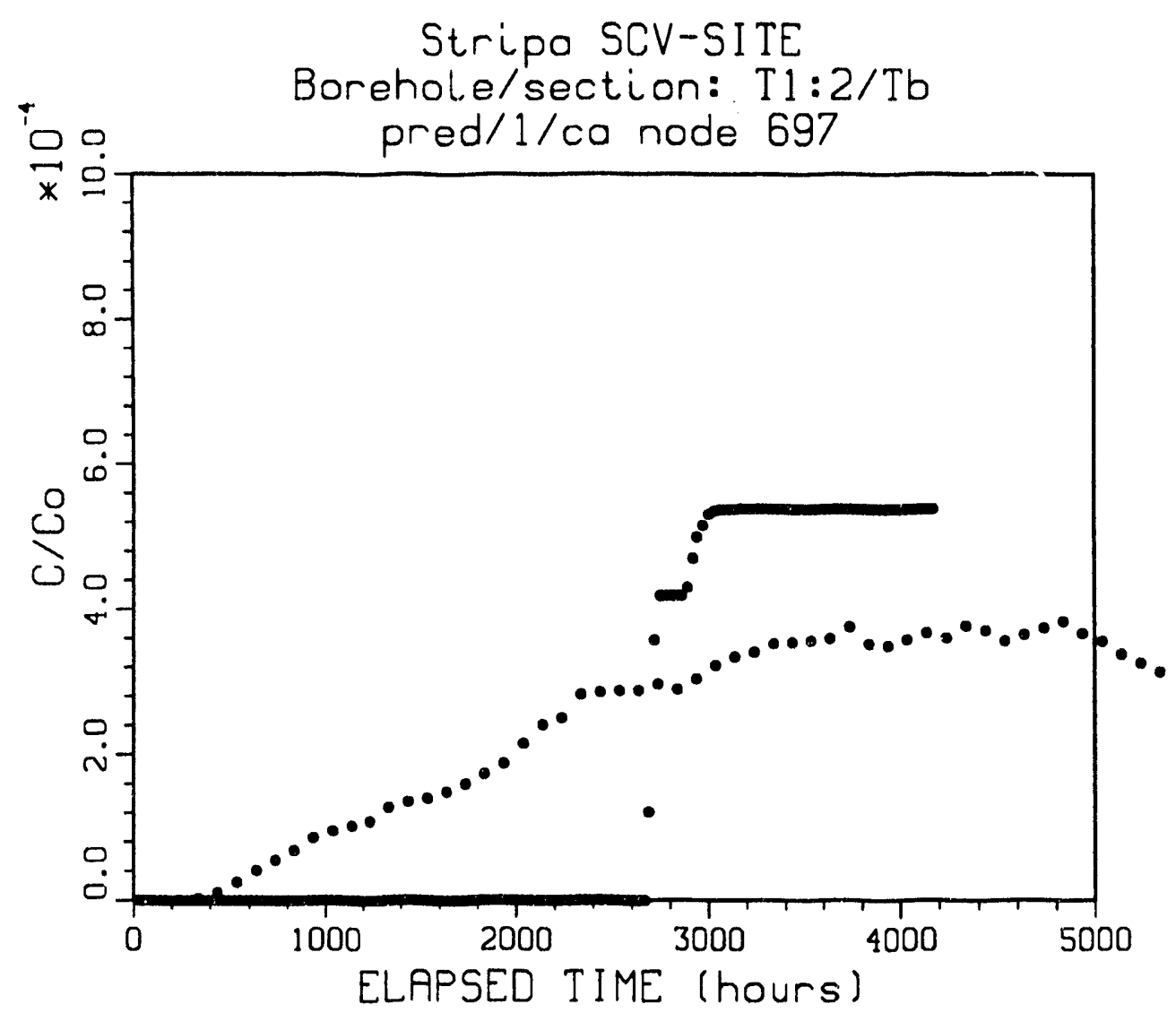

XBL 921-5224

Figure 7.3. Predicted breakthrough into the Validation Drift from $\mathrm{T} 1$ for the co-annealed configuration. The data is shown by dots, and the simulation by the solid line. 


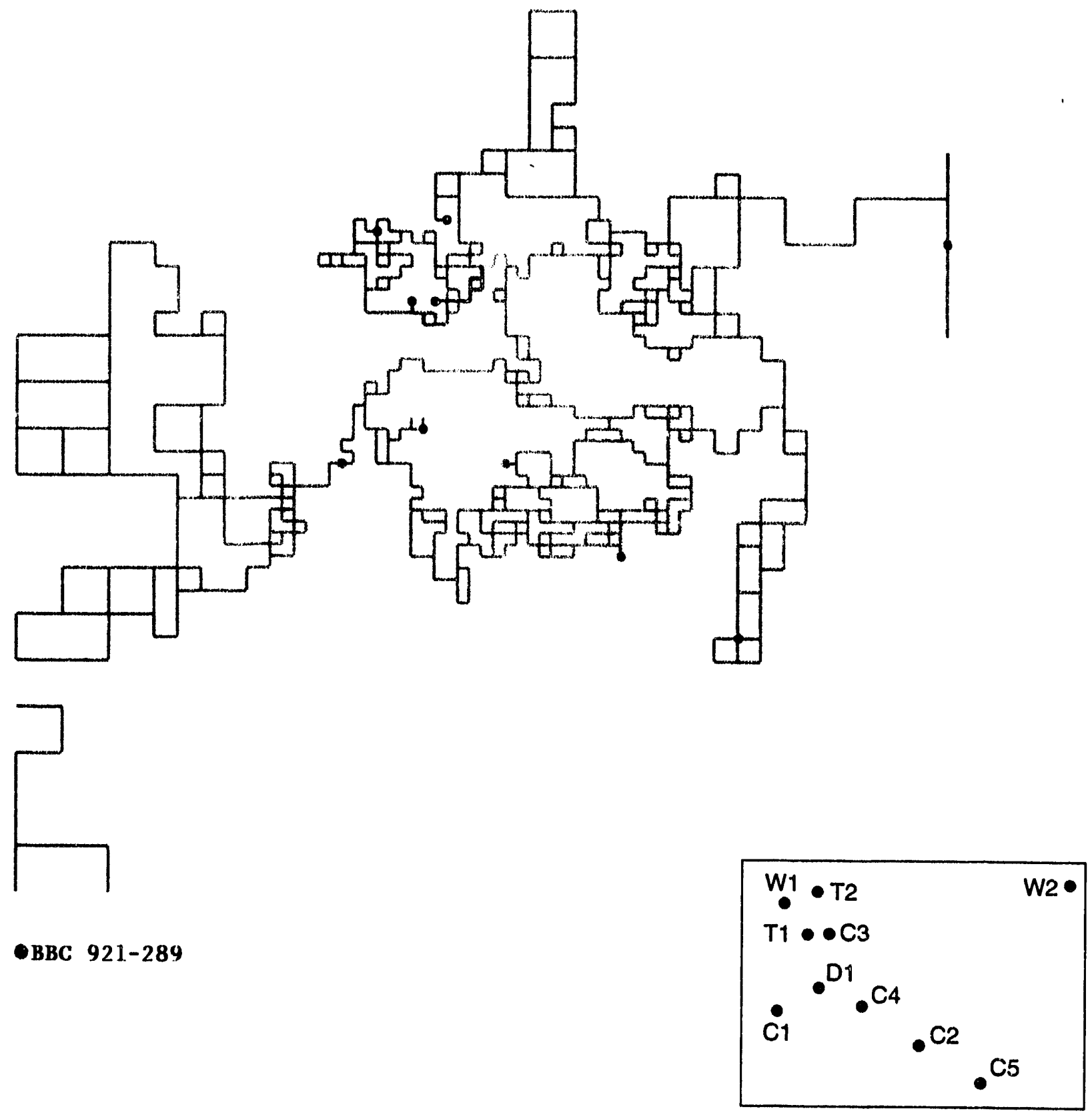

Figure 7.4. Snapshot of concentration for Case 1, injection in $\mathrm{T} 1$ for the co-annealed configuration. 
concentrations were much higher than the predicted values and are not shown on the plot. Figure 7.6 shows that some of the tracer escapes around the drift to the Z-shaft. The actual breakthrough time was about $200 \mathrm{hrs}$, much less than predicted by this simulation.

Figure 7.7 shows the predicted breakthrough to the drift for injection into $\mathrm{T} 1$ for the coannealed configuration. The breakthrough time is almost exactly the same as that observed and the predicted maximum concentration is ligh, as expected from RSI, but well within an order of magnitude. Figure 7.8 shows the snapshot of concentration.

\subsubsection{Case 3: Transport from $\mathrm{C2}$}

Figure 7.9 shows the snapshot of concentration for injection in C2 for the C1-2 configuration. All of the tracer is captured by the Z-shaft and there is no breakthrough to the drift. The actual breakthrough did occur at about $200 \mathrm{hrs}$.

Figure 7.10 and 7.11 give the breakthrough and snapshots respectively for injection into $\mathrm{C} 2$ in the co-annealed configuration. The predicted breakthrough time and maximum concentration are extremely close to that predicted.

\subsection{Case 4: Transport to $T 1$ and the Drift from $T 2$}

Figure 7.12 shows the breakthrough at $\mathrm{T} 1$ from $\mathrm{T} 2$ in the $\mathrm{C1}-2$ configuration. Taking into account the background levels of tracer, actual breakthrough was at about $50 \mathrm{hrs,}$ very close to that predicted. Recall that this test had poorly controlled hydraulic conditions. Further, the value of inflow to $\mathrm{T} 1$ used in this simulation was significantly too high. This explains why in Figure 7.13, no tracer reaches the drift: it all shows up in T1 which is a much stronger sink than in reality. Figures 7.14 and 7.15 show essentially similar behavior for the co-annealed configuration.

\subsection{Conclusions}

Based on these calculations the following estimates of arrival time and maximum concentration can be made for the tracer tests and compared to the actual data: 


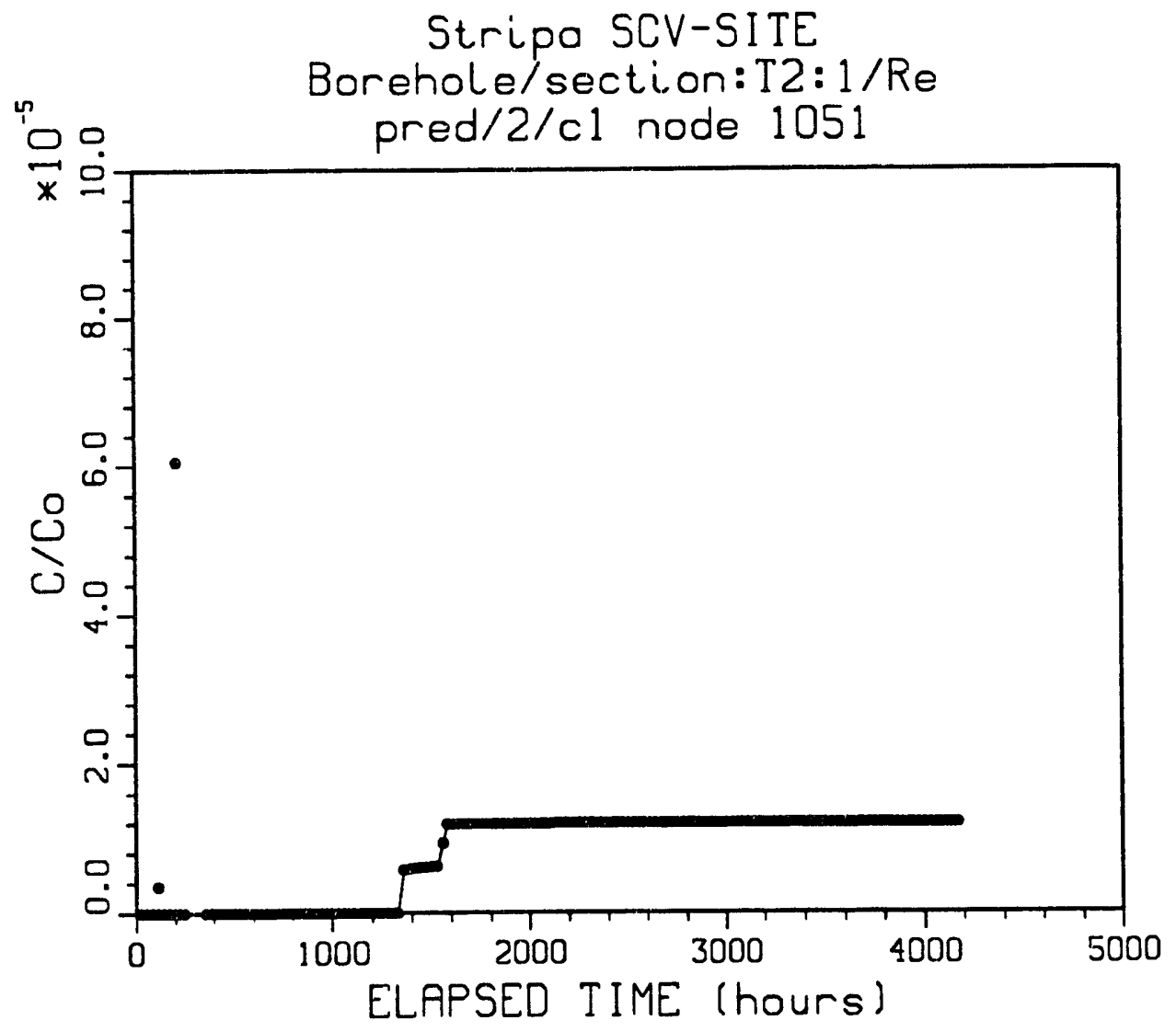

XBL 921-5225

Figure 7.5. Predicted breakthrough into the Validation Drift from $\mathrm{T} 2 \mathrm{fo}$ the $\mathrm{C} 1-2$ configuration. The data is shown by dots, and the simulation by the solid line. 


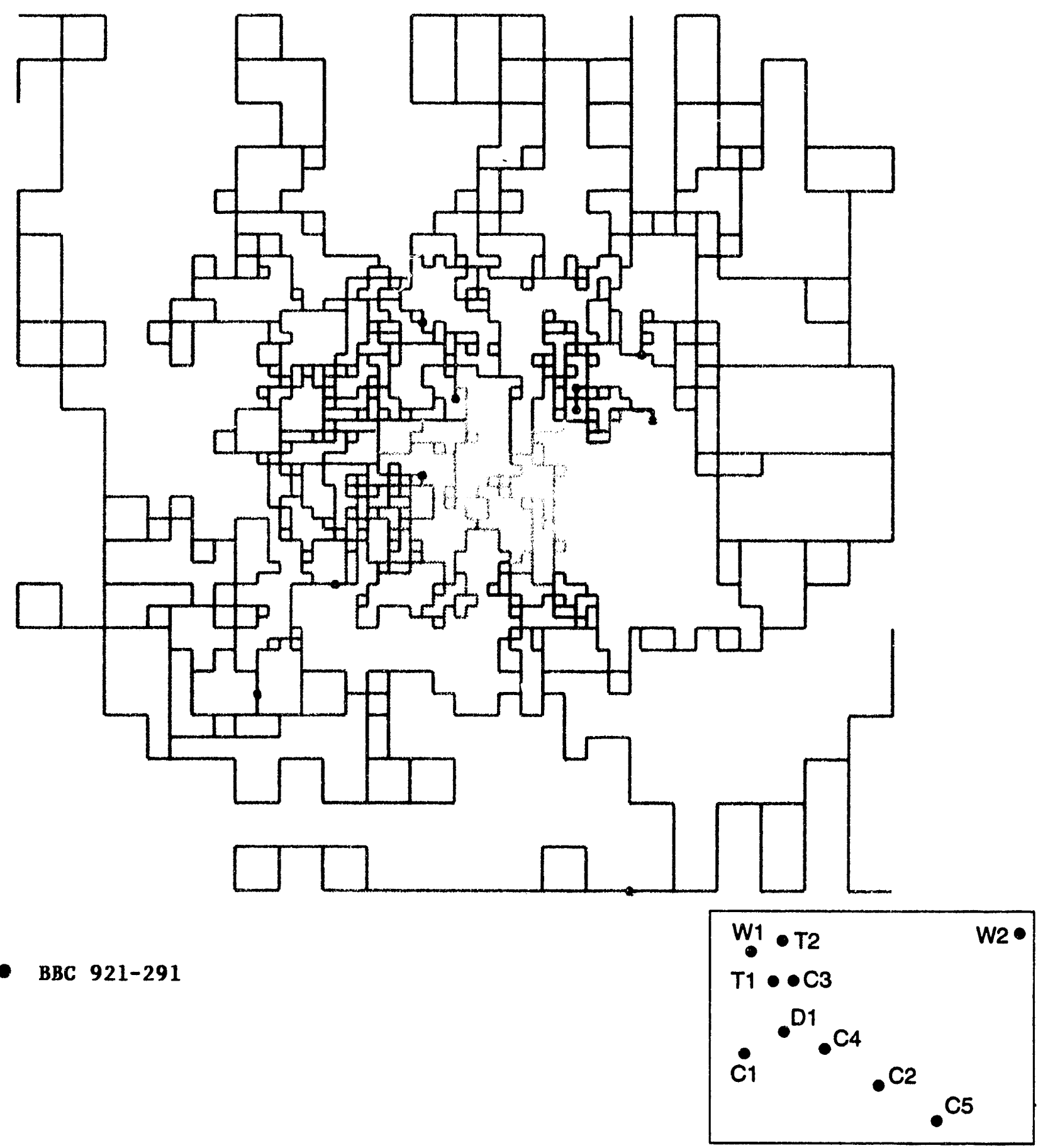

Figure 7.6. Snapshot of concentration for Case 2, injection in $\mathrm{T} 2$ for the $\mathrm{C} 1$ configuration. 


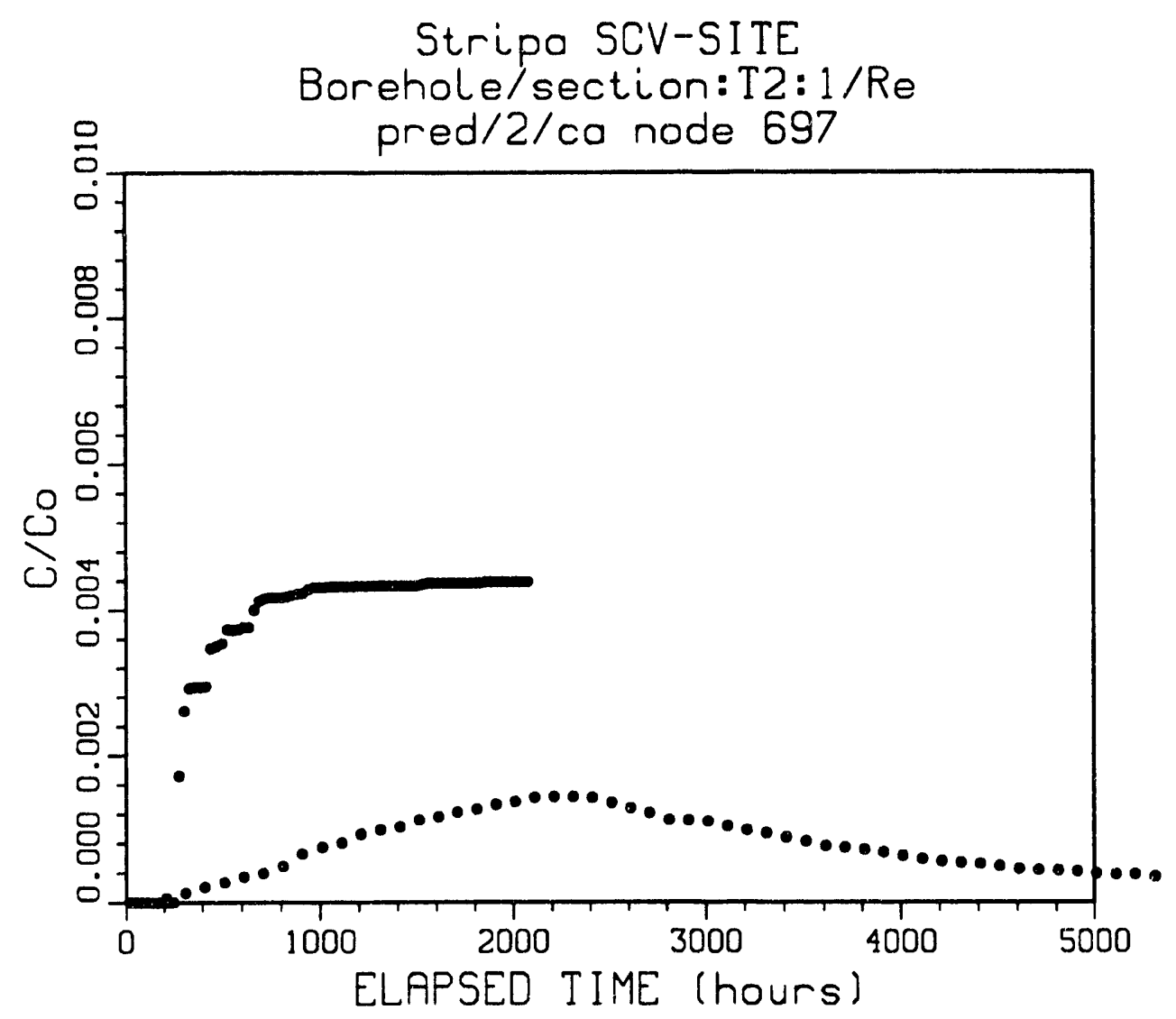

XBL $921-5226$

Figure 7.7. Predicted breakthrough into the Validation Drift from $T 2$ for the co-annealed configuration. The data is shown by dots, and the simulation by the solid line. 

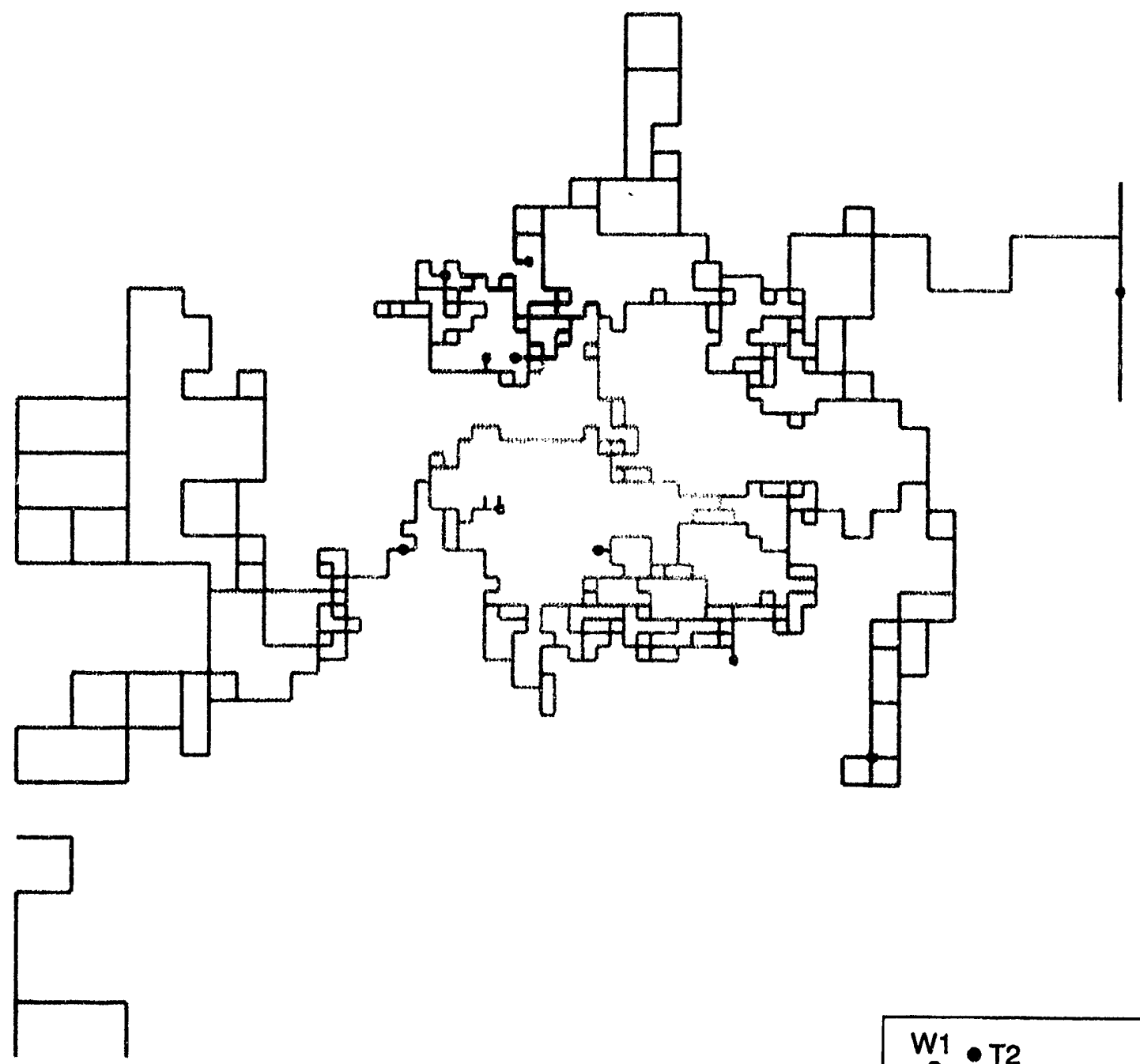

- ВBC 921-293

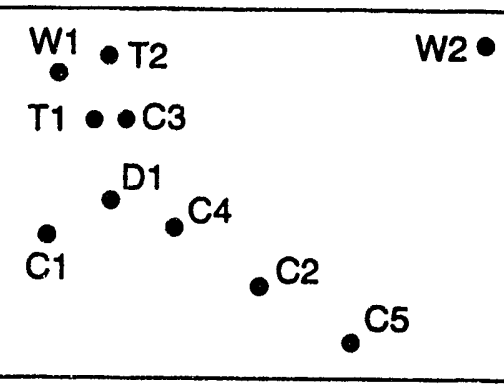

Figure 7.8. Snapshot of concentration for Case 2, injection in $T 2$ for the co-annealed configuration. 


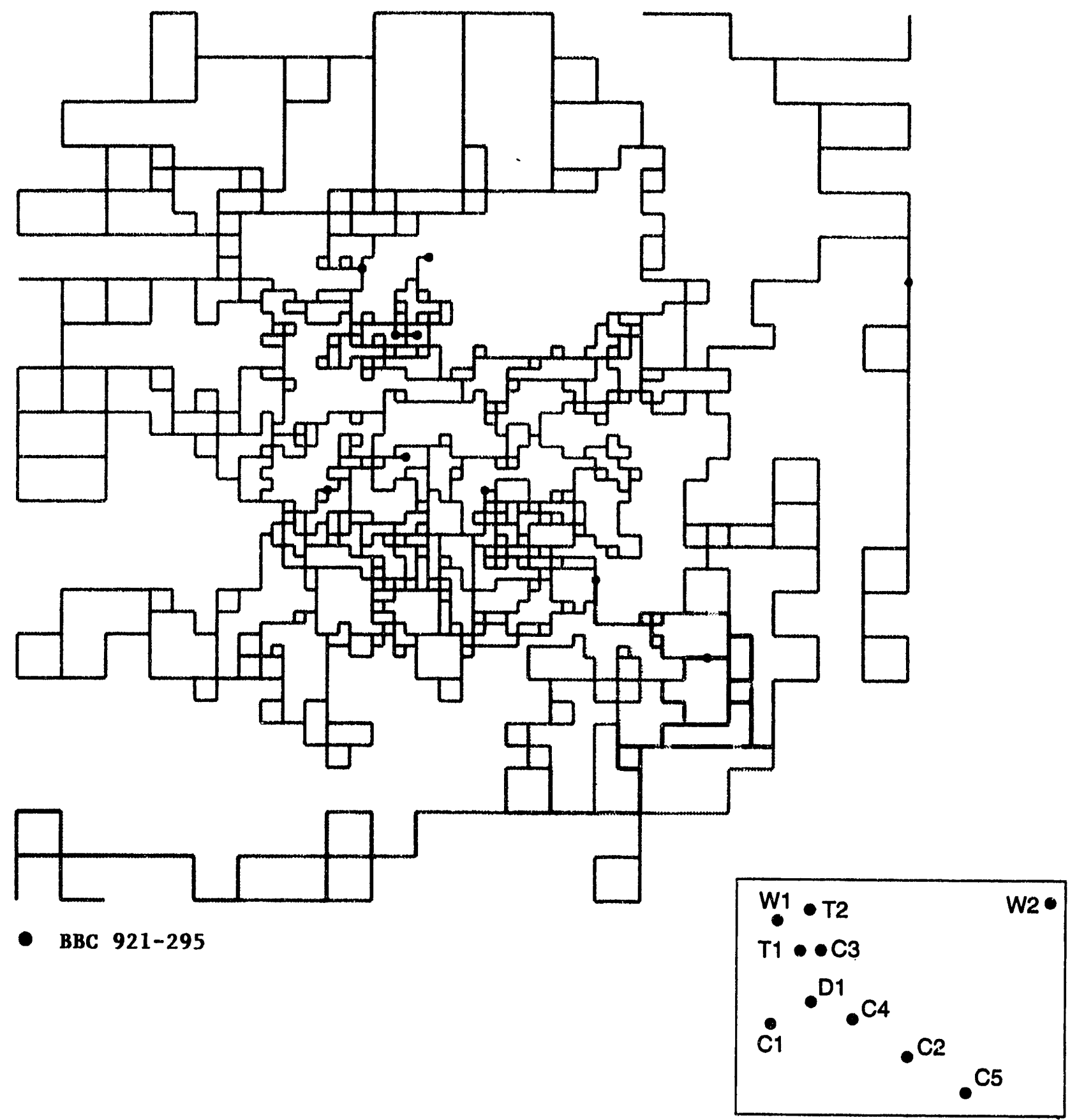

Figure 7.9 Snapshot of concentration for Case 3, injection in $C 2$ for the $C 1$ configuration. 


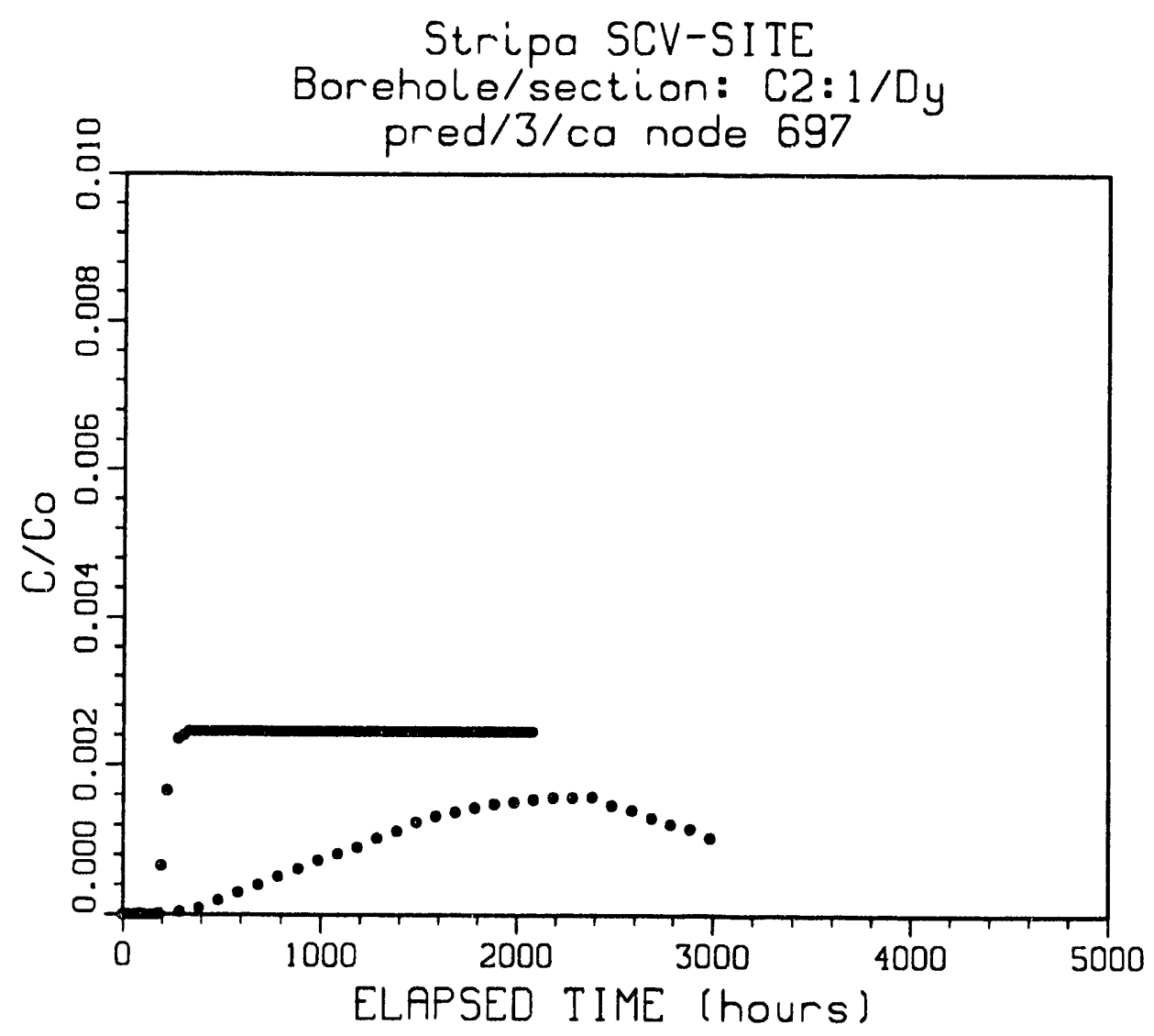

XBL 921-5227

Figure 7.10. Predicted breakthrough into the Validation Drift from $\mathrm{C} 2$ for the co-annealed configuration. The data is shown by dots, and the simulation by the solid line. 


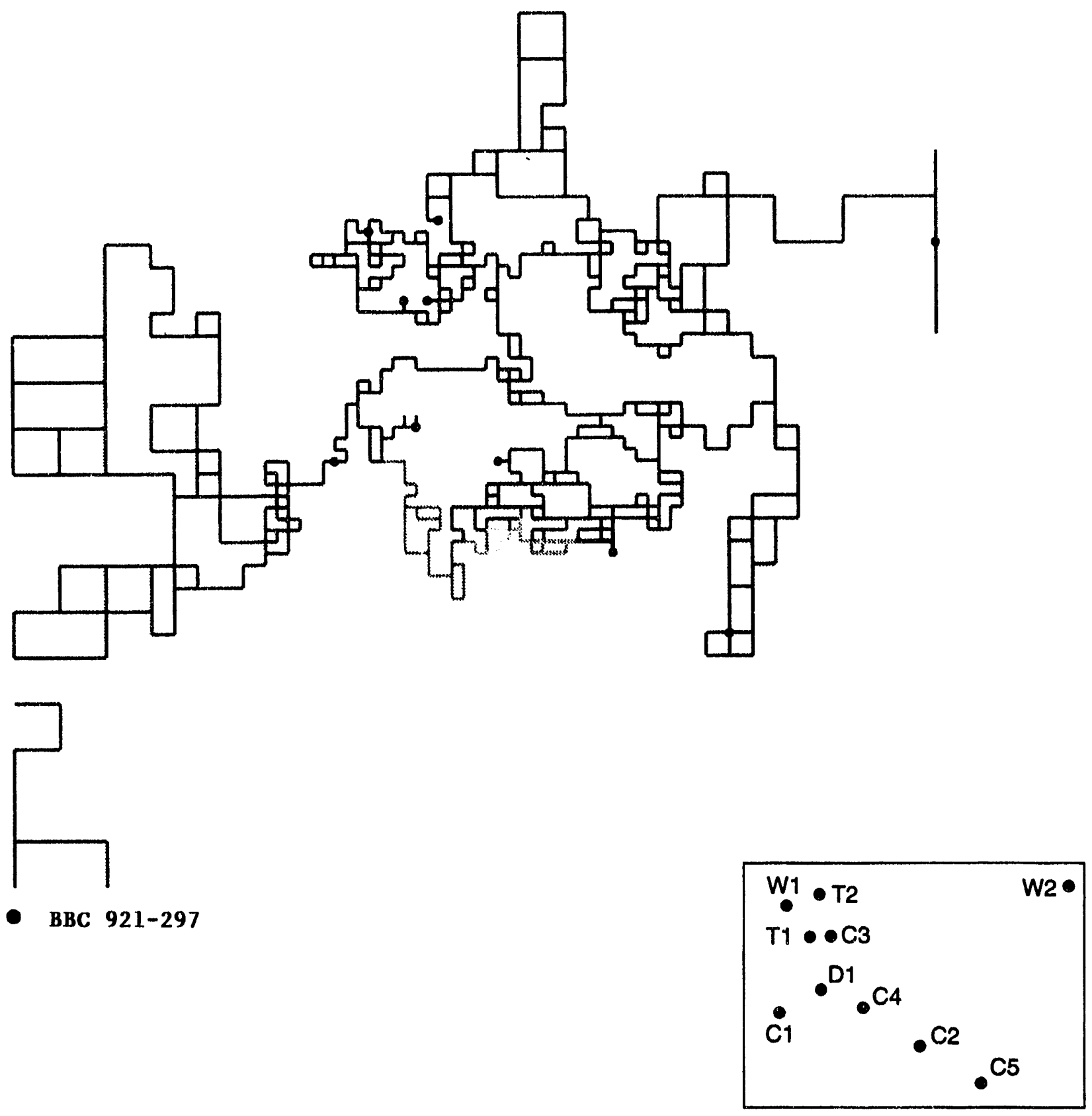

Figure 7.11. Snapshot of concentration for Case 3, injection in $\mathrm{C} 2$ for the co-annealed configuration. 


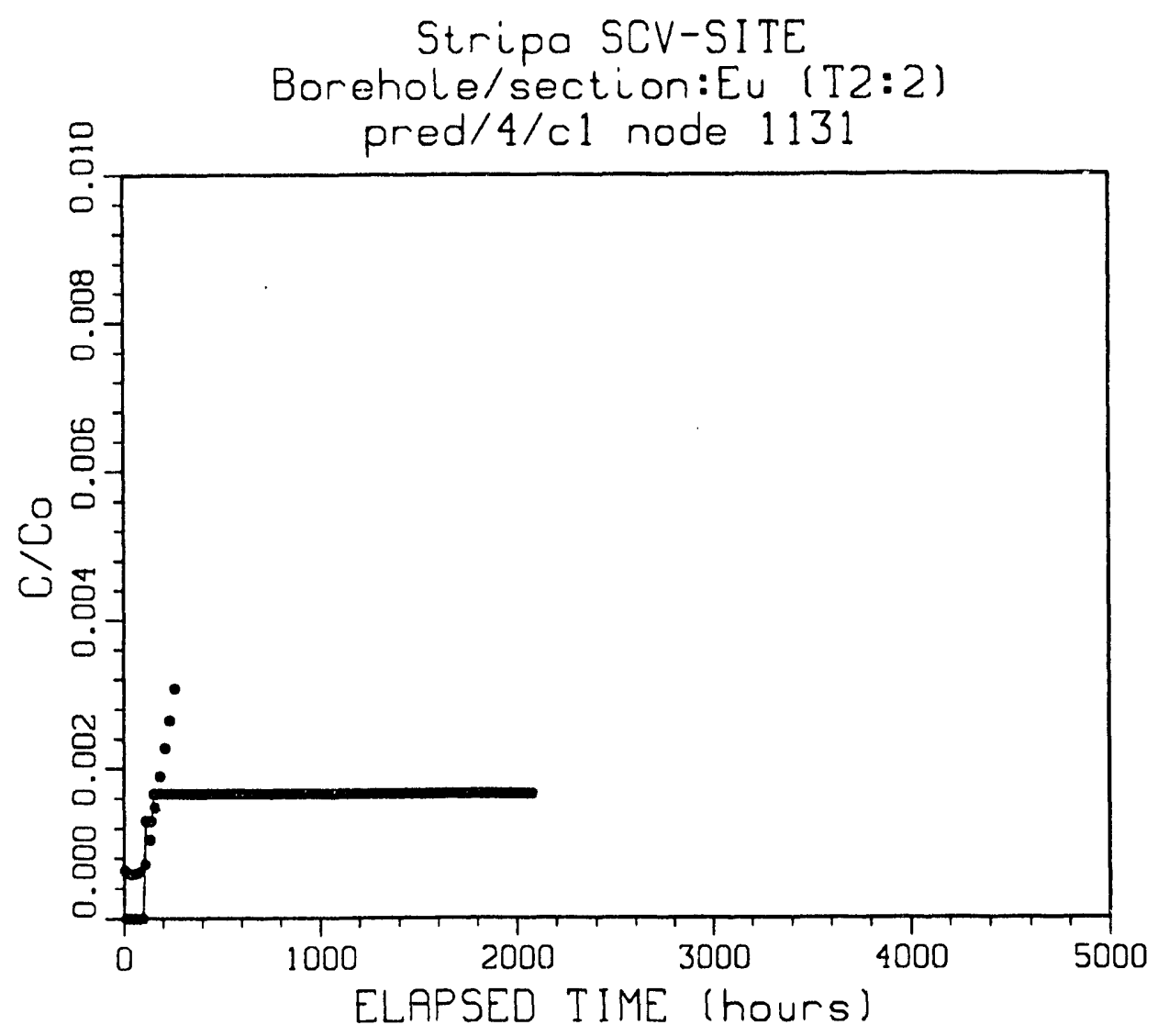

XBL $921-5228$

Figure 7.12. Predicted breakthrough to $\mathrm{T} 1$ from $\mathrm{T} 2$ in the configuration $\mathrm{C} 1-2$. The data is shown by dots, and the simulation by the solid line. 


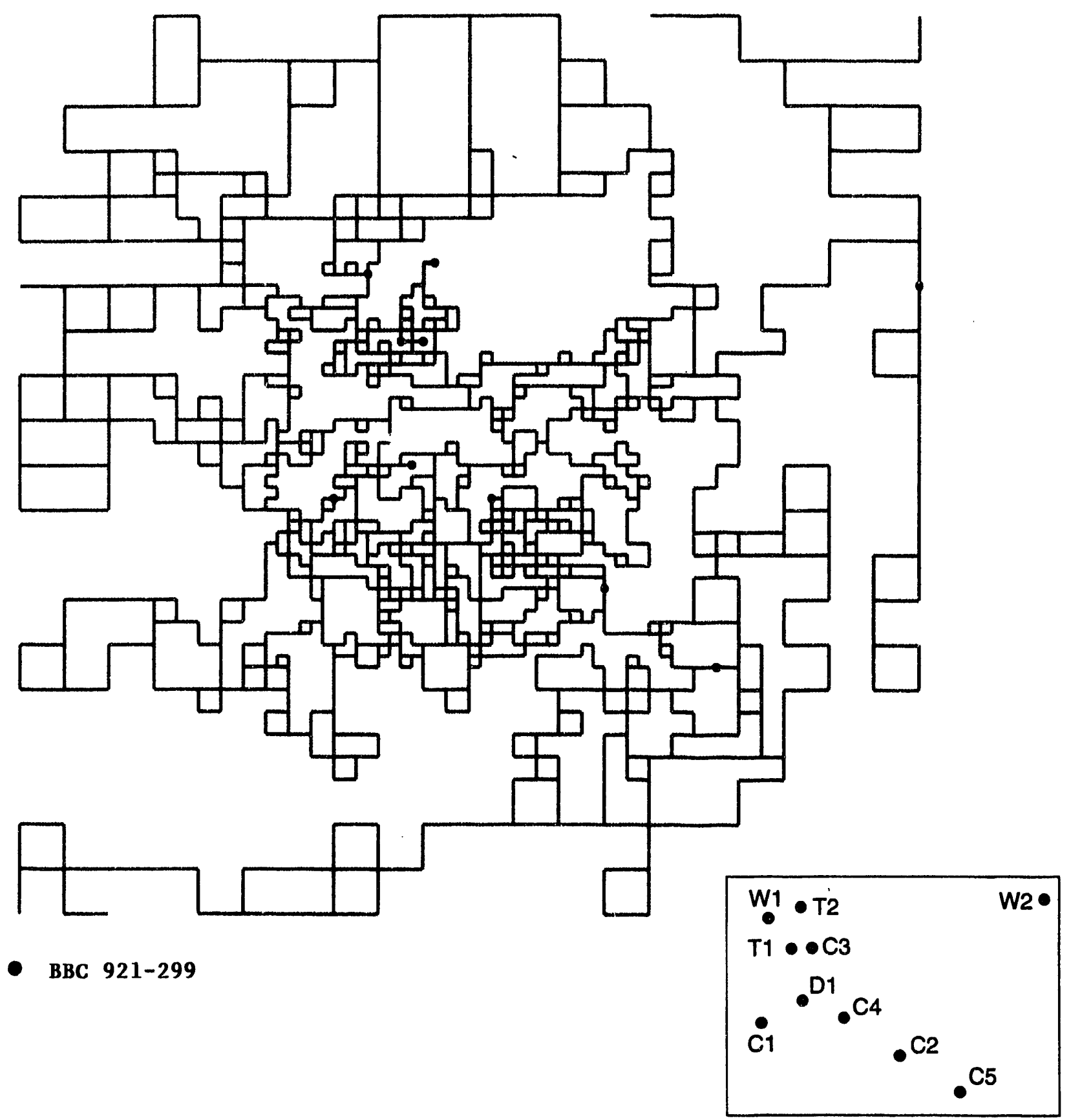

Figure 7.13. Snapshot of concentration for Case 4, injection into $\mathrm{T} 2$ and collection in $\mathrm{T} 1$ and the drift for the $\mathrm{C} 1$ configuration. 


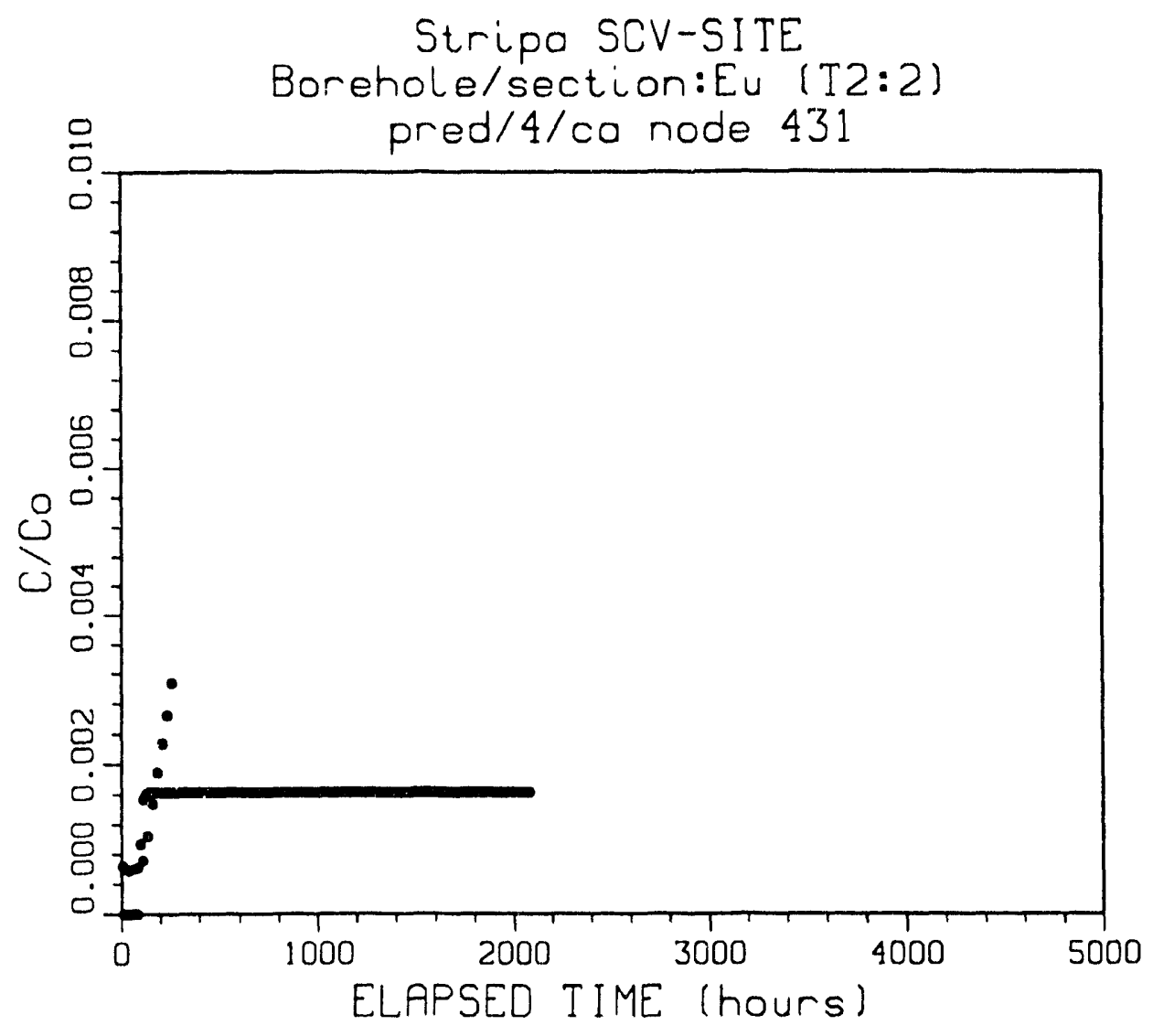

XBL $921-5229$

Figure 7.14. Predicted breakthrough to $\mathrm{T} 1$ from $\mathrm{T} 2$ in the co-annealed configuration. The data is shown by dots, and the simulation by the solid line. 


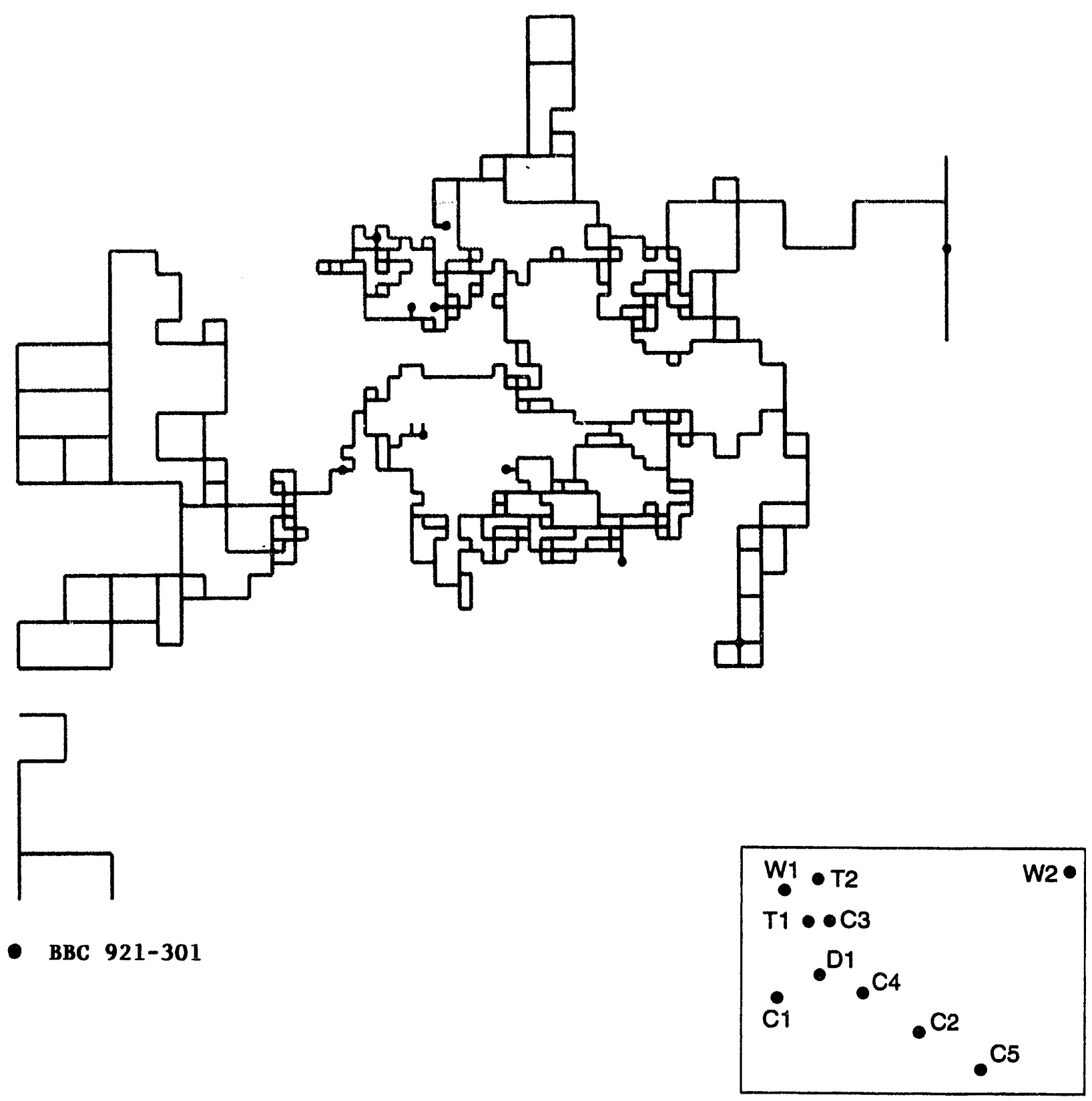

Figure 7.15 Snapshot of concentration for Case 4, injection into $\mathrm{T} 2$ and collection in $\mathrm{T} 1$ and the drift for the co-annealed configuration. 
Table 7.1. Estimates of first arrival and maximum concentration for the tracer tests.

\begin{tabular}{|c|c|cc|cc|}
\hline Case & Source & \multicolumn{2}{|c|}{ First arrival (hrs) } & \multicolumn{2}{|c|}{ Maximum C/Co } \\
& & prediction & data & prediction & data \\
\hline \hline 1 & T1:2 & 800 to 2800 & 400 & 0.0001 to 0.0005 & \\
2 & T2:1 & 200 to 1400 & 200 & 0.0001 to 0.0004 & 0.002 \\
3 & C2 & 200 to never & 300 & 0 to 0.002 & 0.002 \\
\hline 4 & T2 & 100 (to T1) & 100 & 0.0018 & $?$ \\
4 & T2 & never (to VD) & 200 & 0 & $?$ \\
\hline
\end{tabular}

All of the simulated breathrough curves are too steep. This means that the tracer was transported essentially by plug flow. Plug flow occurs because the network of conductors all have the same conductance. If the conductance were to vary, travel times in different paths would also vary and the breakthrough curve would be shallower. This is especially evident in Case 4 where there was very little difference between the estimates for $\mathrm{C1-2}$ and the co-annealed configurations, probably because the models do not have very much resolution on the scale of the distance between these two wells. Thus, this estimate is probably more dependent on the level of discretization than the others.

The estimates of travel time are on the whole better than that for concentration: All travel times are within an order of magnitude. This means that the use of interference data to determine the configuration of the lattice is more successful at helping to determine travel time than dilution. Many of the problems with dillution stem from a poor understanding of boundary conditions. In the model, tracer can only go to the sinks that are specified and the relative strength of these sinks is a critical factor in determining concentration.

The comparison of the Case 4 prediction with data is probably not very useful. The test was not conducted under controlled conditions and the simulation incorrectly represented the hydraulic role of $\mathrm{T} 1$. 


\subsection{CONCLUSIONS AND RECOMMENDATIONS}

A series of tracer simulations were made for transport in a fracture zone based on two equivalent discontinuum models, called $\mathrm{Cl}-2$ and the co-annealed configurations (Long et al. 1992). The first radar/saline case simulated transport from one borehole to a collection of boreholes, the D-holes which intersect the fracture zone. The actual breakthrough curve was used to calibrate the model by changing the ratio of flow to velocity in the conductive elements of the model to match the breakthrough curve. Only advective dispersion of tracer was allowed. This calibrated model was then used to predict a second radar/saline tracer experiment, this time from a borehole to a drift located where the collection of boreholes used to be. The calibrated model did not too badly calculating arrival time but significantly over estimated the maximum $C / C_{0}$. Then a series of tracer tests to the drift and to a borehole near the drift were simulated. Data from these tests is not yet available for comparison.

The simulations of RSI can be exmained to see how well the two configurations are able to model tracer dispersion as a solely advective process. (No dispersion coefficient was used in the simulations.) The simulated RSI breakthrough curve for the C1-2 configuration over-estimates dispersion and the co-annealed configuration underestimates it. Too many pathways are available in the C1-2 case and too few in the co-annealed case. The indication is that repeated annealing of the interference data may produce a reasonable ensemble estimate of advective dispersion. However, this was beyond the scope of investigation.

In the models, the change in boundary conditions from RSI to RSII had the effect of increasing the maximum $C / C_{0}$. In data, the excavation left the maximum $C / C_{0}$ relatively unchanged. The increase in $\mathrm{C} / \mathrm{C}_{0}$ from the simulation of RSI to RSII is explainable because the injected water is a greater proportion of the inflow to the drift than the inflow to the D-holes. However, the fact that the same trend is not observed in the data is interesting. The second 
radar/saline experiment differed from the first in that 1) the drift had been excavated, 2) the remaining portion of the D-holes were left open and 3) higher injection pressures were needed to obtain the same saline injection rate.

The effect of the drift excavation was modeled as a change in boundary conditions but the actual effect of excavating the drift has a complex effect on the hydrologic regime. Long et al. (1991a) have suggested that depressurization of the water entering in the drift causes de-gassing which creates two-phase flow near the drift. The two-phase flow could account for an inflow rate to the drift which is about 4 times lower than expected. This disturbed zone probably has an effect on the tracer transport that is difficult to predict. Gvirtzman and Gorelick (1991) recently performed a study showing that transport in unsaturated regimes is greatly affected by anion exclusion. Anion exclusion increases the apparent dispersion coefficient by only allowing tracer to travel in the fast flow paths and the effect is enhanced in unsaturated systems. A larger dispersivity could cause more mixing and lower $\mathrm{C} / \mathrm{C}_{0}$. Further, some of the tracer might be left in the walls of the drift as inflowing water evaporates. Whether either of these phenomena have any relevance to the Validation Drift is not obvious, but it does point out that transport is affected by the excavation in a way that is not known.

In RSII, the remaining D-holes were left open, allowing the B-zone to drain. These intervals were not open during RSI. However, the remaining D-holes were not represented in the model because they are out of the plane of the H-zone. It could be that RSII values of $C / C_{0}$ are affected by a loss of tracer towards the remaining D-holes. This would account for the predicted values being too high.

The Neretnieks tracer test simulations compare well with some of the data. Based on the simulations, cases where tracer does not show up at the drift at all would be expected and this did not happen. The root cause may be that the Z-shaft is simply not a significant sink. Given the limited number of realizations available, the number of cases where travel time is predicted precisely is encouraging in that it indicates that interference data is useful in predicting flow paths. The amount of dillution is strongly controlled by the boundary conditions and these were poorly 
known. The travel times are fairly well predicted, especially given the limited number of realizations available. In all, the this work shows that the ability to model tracer transport is strongly controlled by the accuracy of the flow model.

\subsection{Recommendations}

The most important recommendation of this report is that future studies of tracer transport in fracture netwiorks should be done unjer controlled boundary conditions, controlled source and sink conditions, and steady flow. The SCV project has shown clearly that the hydrology of excavations is incompletely understood. Coupling these two problems makes it very difficult to interpret the experiments.

It would be preferable to carry out a transport investigation by first conducting a series of well controlled, repeatable interference tests which can demonstrate hydraulic connections. 'Then a tracer can be added to the flow system at steady state in series of convergent tests from intervals known to be connected to the hydraulic sink. These should be repeated at different steady flow rates. The emphasis should be on simple, well controlled, repeatable tests. Under such controlled conditions, it will be much easier to determine the best methodology for parameterizing transpoit models in fractured rock.

The modeling technology as described here may not be sufficient for modeling transport. Some steps have been taken towards developing a transpor model and the results are encouraging. In this report, one parameter has simply been calibrated to one breakthrough curve with a poor statistical sample oi possible configurations. The first improvement would be to create a larger sample of configurations, but more importantly, research is required to find the best way to determine the additional parameters that control transport. 


\subsection{REFERENCES}

Black, J., O. Olsson, J. Gale, D. Holmes, 1991. Site Characterization and Validation, Stage 4. Preliminary Assessment and Detail Predictions, Stripa Swedish Nuclear Fuel and Waste Management Co., Stockholm, Sweden. Report ID No. TR 91-08.

Davey, A., K. Karasaki, J. C. S. Long, M. Landsfeld, A. Mensch and S. Martel, 1989. Analysis of the Hydraulic Data of the MI Experiment, Report No. LBL-27864, Lawrence Berkeley Laboratory, Berkeley, CA.

Gvirtzman, H. and S. M. Gorelick, 1991. Dispersion and Advection in Unsaturated Porous Media Enhanced by Anion Exclusion, Nature, 352, August 29.

Karasaki, K., 1986. Well Test Analysis in Fractured Media, Ph.D. Thesis, Deparment of Materials Science and Mineral Engineering, University of California at Berkeley.

Karasaki, K., 1987. A New Advection-Dispersion Code for Calculating Transport in Fracture Networks, Report No. 22090, Earth Science Division 1986, Lawrence Berkeley Laboratory, Berkeley, CA.

Long, J., K. Karasaki, A. Davey, J. Peterson, M. Landsfeld, J. Kemeny and S. Martel, 1991. An Inverse Approach to the Construction of Fracture Hydrology Models Conditioned by Geophysical Data-An Example from the Validations Exercises at the Stripa Mine, Int. J. Rock Mech, Min. Sci and Geomech. Abstr. 28, (2/3), 121-142.

Long, J. C. S., A. D. Mauldon, K. Nelson, S. Martel, P. Fuller and K. Karasaki, 1992. Prediction of Flow and Drawdown for the Site Characterization and Validation Site in the Stripa Mine, Report No. LBL-31761, Lawrence Berkeley Laboratory, Berkeley, CA.

Olsson, O., J. H. Black, J. E. Gale and D. C. Holmes, 1988. Site Characterization and Validation, Stage 2-Preliminary Predictions,

Olsson, O., P. Andersson, E. Gustafsson, 1991a. Site Characterization and Validation - Monitoring of Saline Tracer Transport by Borehole Radar Measurements - Phase 1, Swedish Nuclear Fuel and Waste Management Co., Stockholm, Sweden. Report ID No. TR 91-09.

Olsson, O., P. Andersson, E. Gustafsson, 1991b. Site Characterization and Validation - Monitoring of Saline Tracer Transport by Borehole Radar Measurements - Final Report. Swedish Nuclear Fuel and Waste Management Co., Stockholm, Sweden. Report ID No. TR 91-18. 

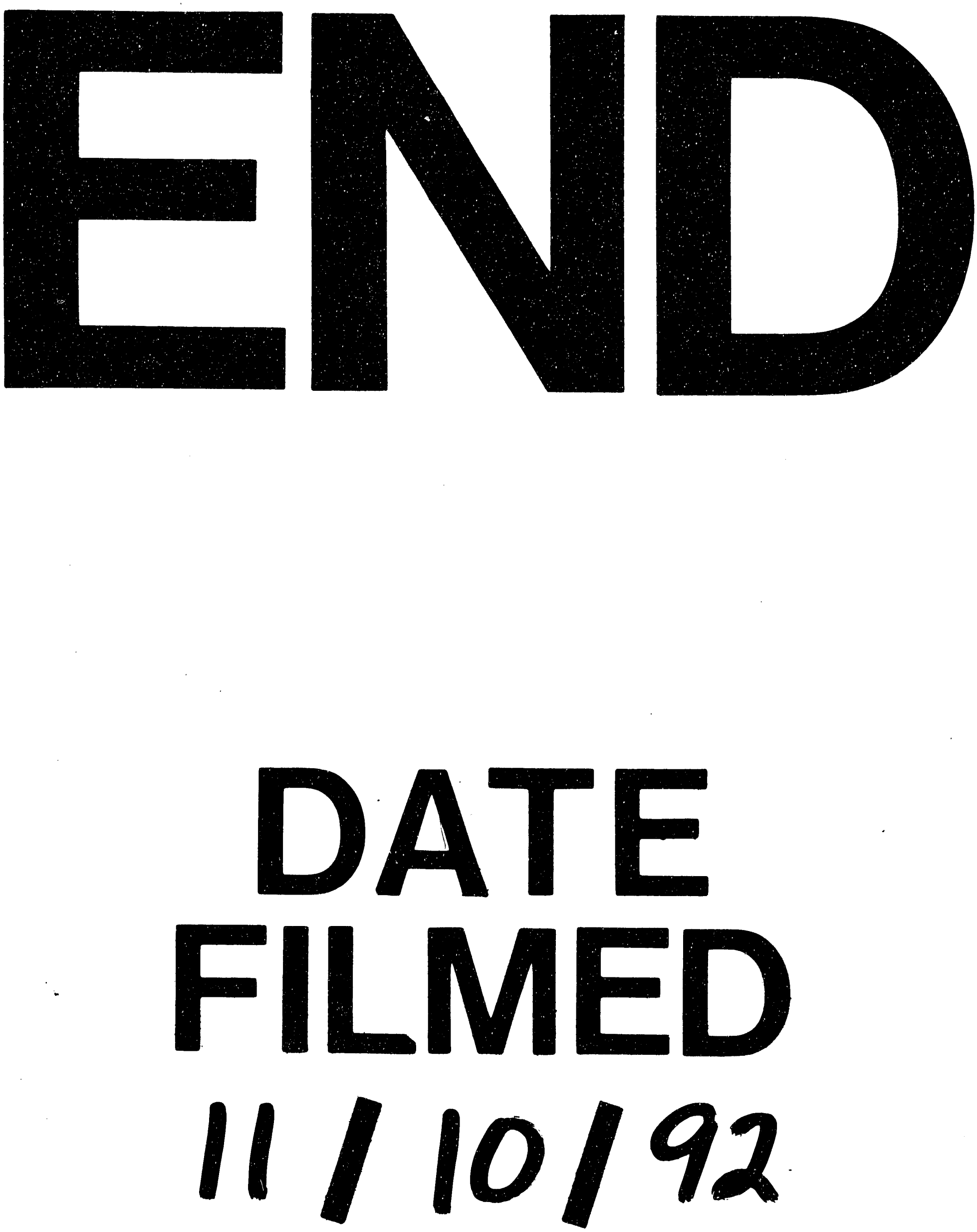

$p$ 
